\title{
Return of the narrative: studies on transportation in social media
}

Citation for published version (APA):

van Laer, T. (2011). Return of the narrative: studies on transportation in social media. [Doctoral Thesis, Maastricht University]. Maastricht University. https://doi.org/10.26481/dis.20110617tl

Document status and date:

Published: 01/01/2011

DOI:

10.26481/dis.20110617t|

Document Version:

Publisher's PDF, also known as Version of record

\section{Please check the document version of this publication:}

- A submitted manuscript is the version of the article upon submission and before peer-review. There can be important differences between the submitted version and the official published version of record.

People interested in the research are advised to contact the author for the final version of the publication, or visit the DOI to the publisher's website.

- The final author version and the galley proof are versions of the publication after peer review.

- The final published version features the final layout of the paper including the volume, issue and page numbers.

Link to publication

\footnotetext{
General rights rights.

- You may freely distribute the URL identifying the publication in the public portal. please follow below link for the End User Agreement:

www.umlib.nl/taverne-license

Take down policy

If you believe that this document breaches copyright please contact us at:

repository@maastrichtuniversity.nl

providing details and we will investigate your claim.
}

Copyright and moral rights for the publications made accessible in the public portal are retained by the authors and/or other copyright owners and it is a condition of accessing publications that users recognise and abide by the legal requirements associated with these

- Users may download and print one copy of any publication from the public portal for the purpose of private study or research.

- You may not further distribute the material or use it for any profit-making activity or commercial gain

If the publication is distributed under the terms of Article $25 \mathrm{fa}$ of the Dutch Copyright Act, indicated by the "Taverne" license above, 


RETURN OF THE NARRATIVE 
Copyright 2011 by Tom van Laer

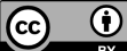

This work is licensed under the Creative Commons Attribution 3.0 Netherlands License. To view a summary of this license, visit http://creativecommons.org/licenses/by/3.0/nl/deed.en or send a letter to Creative Commons, 171 Second Street, Suite 300, San Francisco, California, 94105, USA.

Cover design by Uitgeverij Boekenbent, Barneveld, the Netherlands Printed by CPI Wöhrmann Print Service, Zutphen, the Netherlands

For more information, write to returnofthenarrative@gmail.com

ISBN: 978-90-8570-753-0 


\title{
RETURN OF THE NARRATIVE
}

Studies on transportation in social media

\author{
PROEFSCHRIFT \\ ter verkrijging van de graad van doctor aan de Universiteit Maastricht, \\ op gezag van de Rector Magnificus, prof. mr. G. P. M. F. Mols \\ volgens het besluit van het College van Decanen, \\ in het openbaar te verdedigen \\ op vrijdag 17 juni 2011 om 10.00 uur \\ door
}

Tom van Laer, MA 


\section{Promotor:}

prof. dr. J. C. de Ruyter

\section{Beoordelingscommissie:}

prof. dr. M. G. M. Wetzels (voorzitter)

dr. M. Haenlein (ESCP Europe, Frankrijk)

prof. dr. H. L. G. J. Merckelbach 
And the end of all our exploring Will be to arrive where we started

And know the place for the first time.

-T. S. Eliot (1942, p. 15) 



\section{ACKNOWLEDGMENTS}

Maastricht, 17 April 2011

After having written a dissertation, words of thanks seem more befitting than a preface. First and foremost, I thank my supervisor Prof. Dr. Ko de Ruyter, who asked me at the end of 2007 to write a dissertation. For the most part, working on it has been a great pleasure. His well-chosen and insightful critiques have enabled me to formulate my own position in relation to the views propounded in current literature.

Many thanks also go to Prof. Dr. Martin Wetzels, Dr. Michael Haenlein, ESCP Europe, and Prof. Dr. Harald Merckelbach for their efforts as members of my assessment committee.

I am grateful to Dr. David Cox for his vast network. I have been able to profit from the best of the best in terms of company contacts.

Stimulating exchanges of ideas have taken place with Prof. Dr. Charles Hofacker of Florida State University and Dr. Mathew Chylinski of the University of New South Wales. I have learned much from their expertise and their willingness to share it.

Many at the School of Business and Economics of Maastricht University have inspired and advised me. That goes especially for the academic staff of the Department of Marketing \& Supply Chain 
Management. In particular, the excellent support from our secretaries Nicole Hermans, Eefje Nijst, and Pascalle Prickaerts has had a positive influence on my dissertation.

I gratefully acknowledge the helpful contributions and inspiring interpretations of Prof. Dr. Yoshihisa Kashima, University of Melbourne; Prof. Dr. John Roberts, Australian National University; Prof. Dr. Michael Slater, Ohio State University; Dr. Sonya Dal Cin, University of Michigan; Dr. Jennifer Escalas, Vanderbilt University; Dr. Melanie Green, University of North Carolina; Dr. Raymond Mar, York University; and Dr. Hans Ouwersloot. I thank the Head of Sales Training and the Director of the Sales Management Network \& Consumer Business of the Benelux activities of a global Fortune 100 banking group who provided close and rewarding partnerships. In addition, I gratefully acknowledge the input and assistance of Markus Kösters, MSc, in conducting this research and Berit Knaak, BSc, in meta-coding the studies.

I did not write this dissertation as a leisure activity but as my employment. Every now and then though, this job seemed to get out of hand. Nevertheless, my girl Catherine never withheld emotional support and a firm sense of reality. I express my gratitude to her and my paranymph and friend Jessica for putting up with my peculiarities. Finally, I am grateful to my paranymph and friend Michiel for sticking with me during an engrossing voyage of discovery. 




\section{TABLE OF CONTENTS}

1 GENERAL INTRODUCTION

Practical relevance

Theoretical relevance

1

Narratives

Narrative persuasion

Research questions

2 LOST IN TRANSPORTATION?

Introduction

Conceptual background

Outcomes

Reader moderators

Story moderators

Medium moderators

26

Study approach moderators

Method

Results

39

Discussion

49

Limitations

51 
3 A WALK IN CUSTOMERS' SHOES

Introduction $\quad 55$

Conceptual background 59

Integrity violation ownership $\quad 59$

Transportation 63

Perspective taking 64

Hypotheses development $\quad 66$

Empirical study 69

Pre-test 69

Pre-test results $\quad 75$

Study method 78

Study results $\quad 82$

Discussion 90

Limitations $\quad 91$

4 IN STORIES WE TRUST

Introduction 95

Conceptual background $\quad 99$

Integrity restoration $\quad 99$

Hypotheses development $\quad 102$

Study 1

104

Method 104

Results 106

Discussion 111

Study 2

112

Method 113

Results $\quad 116$

Discussion 119

Study 3

120

Method 120

Results 122

Discussion 124

Limitations $\quad 124$ 
5 THE MEANS TO JUSTIFY THE END

$\begin{array}{ll}\text { Introduction } & 127 \\ \text { Conceptual background } & 132 \\ \text { Identity violation endurance } & 132 \\ \text { Hypothesis development } & 133 \\ \text { Study 1 } & 135 \\ \text { Method } & 135 \\ \text { Results } & 137 \\ \text { Discussion } & 141 \\ \text { Study 2 } & 143 \\ \text { Method } & 144 \\ \text { Results } & 147 \\ \text { Discussion } & 151 \\ \text { Limitations } & 152\end{array}$

6 GENERAL DISCUSSION

Further research suggestions $\quad 158$

Managerial implications 162

Conclusion 169

REFERENCES

INDEX

APPENDICES

SUMMARIES 



\section{1 \\ GENERAL INTRODUCTION}

\section{Practical relevance}

Depending on your age and memory, developments in the modern marketing industry seem radically new or reassuringly old. To me-28 years, passionate Facebook user-the past four years have seemed like the former. I perceive a new deal: Whereas in the past 200 years, media have been pushed to people, today consumers get to be part of the conversation. Using a Facebook-inspired approach, I argue in this dissertation that companies can piggyback on social actions by online users, because people ultimately influence people.

These claims of course echo Katz and Lazarsfeld (Katz, 1957; Katz \& Lazarsfeld, 1955), who argue that marketers do not simply broadcast messages to a passive audience but rather that they target certain "opinion leaders" who then spread, confirm, or negate the marketers' messages through their own "social relationships," whether by word of mouth or personal example. Yet Katz and Lazarsfeld also assume that most of these conversations, and their implicit marketing messages, remain inaudible to firms. The notion that firms might eavesdrop on this chatter became widely conceivable with the rise of weblogs (blogs). Thus Searls and Weinberger (2009, p. 147) could argue that "markets are 
conversations," and blogs can make the conversations transparent.

To the earliest practitioners of this new media form, blogging entailed regular sharing of written stories, later adding photos and videos, about themselves and their experiences, with a few interested friends and relatives. Today lots of Internet users do similar things, though they may not consider it blogging; instead, they only see themselves as updating their profile pages on Facebook, MySpace, or other social media sites. Whether in the form of blogs or profile pages though, these are narratives about who the posters are and what they have been doing.

In turn, narratives of various forms can change people's beliefs and attitudes. Strange (2002) cites Harriet Beecher Stowe's Uncle Tom's Cabin for its important role in galvanizing public opinion against slavery. Abraham Lincoln is reported to have made the same assertion, allegedly calling Stowe when he met her "the little woman who wrote the book that made this great [Civil] war!" (Stowe \& Stowe, 1911, p. 203). More contemporary offline examples include the persuasive power of Latin American telenovelas, which influence family planning choices and enrolment in adult literacy programmes (Slater, 2002a). Despite this widespread belief in the persuasive power of narratives though, we still know little about how these effects occur. In contrast with novels and soap operas, storytelling in social media rarely beguiles readers with happy endings or encourages them to forget that the setting is pure fiction. Moments of joyful release from stress are relatively rare in social media, yet they still may induce persuasive effects. I have conducted research into how and why, which constitute the central questions of this dissertation. 


\section{Theoretical relevance}

\section{Narratives}

For this research, I define "narrative"1 using the least selective definition available in existing research (e.g., Brewer, 1995; Green \& Brock, 2002; Steen, 1998): A narrative is a text that tells the story of an event or sequence of events, leading to a transition from an initial state of affairs to a definitive state. As in art, so in science, the meanderings of a narrative depend on content and format (Brewer \& Lichtenstein, 1982).

Events, characters, and context that provide a causal chain within a specified time span constitute the essential elements of narrative content (Brewer \& Lichtenstein, 1982). First, an event or sequence of events in a narrative involves the transition from one state of affairs into another. I consider three kinds of events. The one most common on blogs features reports of something that has truly happened. A described event also could come across as plausible but still originate in the imagination of the author. Finally, some events are so incredible that there is no question that they originated from fantasy. The sequence of these events, regardless of their type, constitutes the history of the narrative. Second, the events are inextricably bound up with the characters of a narrative. Narrative characters represent part of the state of affairs that precedes the events, and then they play a role in inducing the transition away from this initial state of affairs. Third, the causal chain entails the connections between events and how one event transfers into

${ }^{1}$ The noun "narrative" has its roots in narrare, the Latin verb meaning "to tell a story." 
the next. The causal chain reflects the plot of the narrative (Trabasso \& Sperry, 1985), which can grip readers by relying on four key options: (1) discovery or recognition, (2) reversal, (3) misfortune or suffering, and (4) climax. A climax may be necessary for readers to determine the causal chain. Without the ability to determine the causal chain, the purpose of the narrative remains ambiguous (Graesser \& Kreuz, 1993; Vipond \& Hunt, 1984). Fourth, the time span of each event is definitive. If a time is relatively short, the event is called a crisis; in the case of a slow passage of time, we may speak of development.

Yet even with all these elements of content, a text still may not be a narrative. Rather, narrative requires some typical format that comprises structure and style elements. Structuring a narrative implies that the author has put the events in a particular order. Three basic emotions can guide narrative structures: surprise, curiosity, and suspense. Brewer and Lichtenstein (1982) even delineate different orders of events, depending on the guiding emotion. In a surprising structure, an event necessary to determine the causal chain only gets mentioned later in the narrative. This unexpected order creates a surprising outcome, as exemplified by Brewer and Lichtenstein (1982, pp. 480-481) with the following excerpt:

Charles got up from the chair. He walked slowly toward the window. The window broke and Charles fell dead. The sound of shot echoed in the distance.

The climax (Charles's death) occurs before the revelation of a cause of that event (gunshot), which causes surprise. 
Chronological order instead supports a structure designed to provoke suspense, with the outcome kept a secret until the end. With this structure, recipients remain uncertain what will happen in the next scene, that is, in a state of suspense (Slater, 2002a). Brewer and Lichtenstein (1982, p. 481) provide another example:

The sniper was waiting outside the house. Charles got up from the chair. He walked slowly toward the window. There was the sound of a shot and the window broke. Charles fell dead.

Finally, in a narrative that stimulates curiosity, the outcome gets revealed first, after which readers feel motivated to find out how it came to pass. From that moment, the events can be described in chronological order, so Brewer and Lichtenstein (1982, p. 481) propose:

Charles fell dead. The police came and found the broken glass, etc.

In addition to these types of structure, a narrative format entails its style (Brewer \& Lichtenstein, 1982). According to Biber (1995), narratives mainly use the past tense and thirdperson pronouns, and they should contain dialogue. Readers attend to these dialogues best if the narrator's voice rarely interrupts, though the narrator's voice usually must appear in descriptive or evaluative passages that the author adds to advance the narrative. 


\section{Narrative persuasion}

Prior to 2000, research on persuasive effects was dominated by the so-called dual-process models of persuasion, especially the Elaboration Likelihood Model (ELM, Petty \& Cacioppo, 1986), which attempted to explain how people chose to accept or reject message claims. The determination of the claim's acceptability could result from either careful evaluation of the arguments presented or reliance on superficial cues, such as the presence of an expert. Whether recipients scrutinize a message depends on the extent to which they are motivated and able to process it systematically. As an important mediating variable, this model includes the number and nature of thoughts evoked by the message. If these thoughts are predominantly positive, the recipient's attitude will tend to be more positive as well; if the thoughts are predominantly negative, the resulting attitude will be more negative (Petty \& Wegener, 1998).

Several authors (Escalas, 2007; Green \& Brock, 2000, 2002; Slater, 2002b) assert that though the ELM provides a valid description of analytical persuasion, it cannot encompass narrative persuasion. Analytical persuasion refers to beliefs and intentions developed as a result of processing messages that are overtly persuasive, such as most advertisements, lessons in science books, news reports, and speeches. However, narrative persuasion refers to attitudes and beliefs developed as a result of processing stories that are not overtly persuasive, such as novels, movies, soap operas, or social media messages. Such stories usually are read for entertainment, rather than to gain information. Accordingly, the processes responsible for the persuasive effects of narratives appear markedly different from the processing pattern in the ELM. 
An important distinction between analytical and narrative persuasion is the differential role of engrossment. In analytical persuasion, engrossment results from the extent to which the message has personally relevant consequences for a recipient's money, time, or other resources. If these consequences are sufficiently severe, recipients evaluate the arguments carefully and generate thoughts related to the arguments. Yet as Slater (2002a, p. 171) notes, even though severe consequences for entertainment narratives should be relatively rare, "viewers or readers of an entertainment narrative typically appear to be far more engrossed in the message." This type of engrossment, or "transportation," may be the crucial determinant of narrative persuasion (Escalas, 2007; Gerrig, 1993; Green \& Brock, 2000, 2002; Slater \& Rouner, 2002).

Green and Brock (2000) establish how transportation effects are obtained. They report on four experiments in which they had participants read a printed story under different conditions. They measured the extent to which participants were transported into the story as well as the extent to which participants agreed with story-consistent beliefs. In the first three experiments, a median split was used to distinguish between high and low transportation into the story. Subsequently, this factor was entered into the analysis as a dichotomous variable to assess its effect on storyconsistent beliefs. The effects of transportation on these beliefs were all in the predicted direction but many of them did not reach conventional levels of significance. Furthermore, given that the difference in transportation was observed rather than manipulated, the data were not decisive with respect to the predicted causal relation between transportation and story-consistent belief change. 
In the last of their four experiments, however, Green and Brock succeeded in manipulating the level of transportation. Participants who were instructed to check whether the story contained words and sentences that might prove to be too difficult for fourth-grade level readers felt less transported into the story compared to those who were told simply to read the story attentively. Those participants who read the story attentively proved to agree more with story-consistent beliefs than those who had to check the complexity of the words and sentences. A mediation analysis showed that when the level of transportation was entered into the equation as a covariate, the instruction effect became non-significant, which provided a strong indication that transportation was the factor explaining the persuasive effects of the narrative.

\section{Research questions}

This background suggests that narrative persuasion is conceptually different from analytical persuasion and that the persuasive effect of social media messages may result because readers are transported into their narrative world. In Chapter 2, I conduct a meta-analysis to assess whether this reasoning is accurate, according to the following research question:

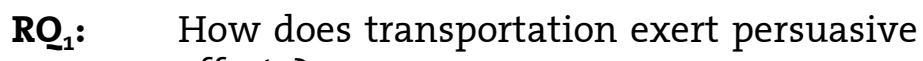
effects?

Many narratives in social media refer to integrity violations for example (Gartner Research, 2007; The Economist, 2006; Woodside, Sood, \& Miller, 2008), such that customer protagonists 
perceive that service employee antagonists have failed to adhere to necessary or acceptable principles and standards (R. C. Mayer \& Davis, 1999). To determine how employees respond to narratives of these focal events (i.e., integrity violations), Chapter 3 proposes a relationship between assuming ownership of the integrity violation and the impact of social media, such that employees may be transported into the customer's narrative. I address the following question through two experiments:

$\mathbf{R Q}_{2}: \quad$ How does transportation affect employees' willingness to assume ownership of integrity violations reported in narrative weblogs?

In this setting, integrity restoration could result from the interplay between the content and the format of the verbal response to a narrative about the violation. This proposition requires a comparison between processing of a narrative and processing of an analytical text. Therefore, in three experiments in Chapter 4, I compare the persuasive impact of a narrative response with the persuasive impact of an analytical response to reports of an integrity violation on a weblog to determine:

$\mathbf{R Q}_{-}: \quad$ How might narrative apologies restore integrity?

Another issue for social media that has yet to receive much empirical attention is the dilemma of cyber harassment. Network attendance declines as a result of cyber harassment, and increased privacy regulations can limit such exits (Krasnova, 
Spiekermann, Koroleva, \& Hildebrand, 2010; Sledgianowski \& Kulviwat, 2009). However, users also might be prone to reject imposed privacy regulation decisions. If they were transported into a narrative report of cyber harassment, these social network users could begin to perceive the privacy regulation decisions as more just. With respect to social media with negative content, two experiments in Chapter 5 address the following question:

RQ- $\quad$ What effect do narratives have on the perceived justice of anti-harassment measures in social media?

In four chapters, I address each question and detail my research into them, all under the supervision of Ko de Ruyter. In addition to presenting the main results of the studies, I offer some answers to the research question that is the foundation for that chapter. In the final chapter, I also offer a broader interpretation of the aggregated results, together with some discussion of my conceptual background, ideas for managers based on these results, and suggestions for further research. 


\section{2 \\ LOST IN TRANSPORTATION?}

A meta-analysis of a decade of narrative transportation research

\section{Introduction}

The potential impact of narrative transportation on beliefs and intentions continues to gain increasing importance in persuasion research (Escalas, 2004a, 2007; Green \& Brock, 2000, 2002; Slater \& Rouner, 2002; Vaughn, 2010). Specifically, according to the transportation effect, when readers lose themselves in a story, their beliefs and intentions change to reflect that story (Green, 2008). Since Green and Brock's (2000) seminal article first catalysed quantitative transportation research, a plethora of studies have investigated narrative accounts, how they transport readers, and how they might change readers' views. Furthermore, recent developments have enhanced the significance of the transportation effect, such as the intentional placement of educational content in entertainment (Moyer-Gusé, 2008) and the rise of hybrid genres such as narrative-based advertising (Chang, 2009), reality television shows (Hall, 2009), and interactive video games (Baranowski, Buday, Thompson, \& Baranowski, 2008). Thus, transportation and narrative persuasion constitute key issues that demand both theoretical and applied research attention (Singhal \& Rogers, 2002). 
Yet despite some notable strides in transportation research, extant literature remains fragmented, in terms of both its conceptual breadth and its empirical findings, as several qualitative reviews highlight (Green, Brock, \& Kaufman, 2004; Moyer-Gusé, 2008; Nabi \& Krcmar, 2004; Vorderer, Klimmt, \& Ritterfeld, 2004; Winterbottom, Bekker, Conner, \& Mooney, 2008). This heterogeneity causes confusion and hinders further development; the research stream in turn suffers from a distinct lack of conceptual clarity, particularly in terms of the reader, story, and medium-that is, the key elements of narrative communication. Perhaps transportation depends on the differences among individual recipients (Dunlop, Wakefield, \& Kashima, 2008b), or perhaps it reflects text quality (Braverman, 2008), or maybe the medium can intensify (Bracken, 2006) or mitigate (Chang, 2009) a sense of transportation. Furthermore, the size of the transportation effect reported differs considerably across studies; not all of them even reach conventional significance levels (e.g., "less than $20 \%$ of all appropriate comparisons", Green \& Brock, 2000, p. 718). A poor understanding of the impact of various methodologies and research design elements may account for the mixed results, in that extant research includes lab studies (e.g., S. E. Morgan, et al., 2005) and field experiments (e.g., Dunlop, et al., 2008b); conducted in both the presence (e.g., Wang \& Calder, 2009) and the absence (e.g., Braverman, 2008) of incentives; that feature specifically young (e.g., Anschutz, Engels, Van Leeuwe, \& van Strien, 2009), elderly (e.g., Petrican, Moscovitch, \& Schimmack, 2008), female (Greenwood, 2007), and male (e.g., Denham, 2004) respondents; and that use stimulus material that varies from television soap operas about organ 
transplants (S. E. Morgan, Movius, \& Cody, 2009) to advertisements for sports shoes (Escalas, 2004a).

Without conceptual or empirical convergence in transportation findings, the preceding review reinforces the need for a comprehensive and quantitative synthesis that can unify and advance the field after a decade of transportation research. Therefore for this research, we have three objectives: (1) develop a conceptual framework that integrates the components and outcomes of transportation, according to a review of extensive contributions to the field; (2) empirically assess a model derived through a meta-analysis by comparing the various methodological and design approaches; and (3) uncover issues that deserve further attention and provide direction for the next decade of research into transportation.

\section{Conceptual background}

Transportation theory originates in ludic reading theory (Gerrig, 1993; Nell, 1988), which suggests that readers find reading entertaining for two reasons. First, they might imagine the actions performed by a story character and thereby construct a mental simulation of the events. Second, they can compare themselves to the character and claim positive attributes the character possesses. Gerrig (1993) offers a qualitative observation that readers also may be changed by their reading experience, after they return to reality. Such affective or cognitive alterations could include beliefs and intentions (Green \& Brock, 2000), such that readers who become engrossed in their reading experience tend to accept the story's persuasive message or subtext, as well as the beliefs and behaviours that the characters exhibit. In contrast, if 
readers do not lose themselves in a story (i.e., are not transported), they may respond negatively to a story or its characters and reject the worldview provided by the narrative.

Transportation theory further suggests that exposure to a story can activate empathic feelings and vivid images for the reader. These transportation components affect a person's propensity toward transportation and susceptibility to persuasion, along with attributes that define the recipient, text, and context (Green, 2008; Green \& Brock, 2002). To further understanding of how transportation exerts persuasive effects, we define it precisely, according to three key features. First, transportation requires that readers process stories, which can be conveyed by various media, including novels, movies, and soap operas. Second, readers get transported through two main components: empathy and mental imagery (Green \& Brock, 2002). Empathy implies that readers develop positive feelings toward characters portrayed in the narrative and thus vicariously experience a connection with those characters' values and outcomes. Mental imagery instead means the reader generates vivid images of the narrative events, such that they feel as though they are experiencing the events themselves. Third, when transported, readers lose track of reality in a physiological sense. This component relates transportation to similar phenomena, such as absorption (Tellegen \& Atkinson, 1974) and optimal experience or "flow" (Csikszentmihalyi, 1992). Nevertheless, we note several subtle but critical differences. In particular, transportation is an engrossing temporary experience (Sestir \& Green, 2010), whereas absorption refers to a personality trait or general tendency to be immersed in life experiences. Furthermore, people engage in 
transportation experiences for fun or entertainment, whereas optimal flow experiences focus on a desire to be challenged.

In accordance with our definition, we identify belief changes, behavioural intentions, and affective and cognitive responses as potential outcomes of transportation. Most investigations of beliefs and intentions stem from persuasion research, which suggests that the affective and cognitive nature of responses are important mediating variables (e.g., Petty \& Cacioppo, 1986). Thus when a person is emotionally affected by the events a narrative depicts, it "may lead to at least temporary acceptance of values and beliefs that represent a shift from the individual's existing beliefs" (Slater \& Rouner, 2002, p. 177). Cognitive responses instead imply rational thoughts, which may be positive, negative, or neutral (or irrelevant). If readers generate more positive than negative thoughts, a persuasive attempt should succeed.

We summarize the path to the transportation effect as a simplified conceptual model in Figure 2-1 before we develop and describe our model variables in greater detail. 


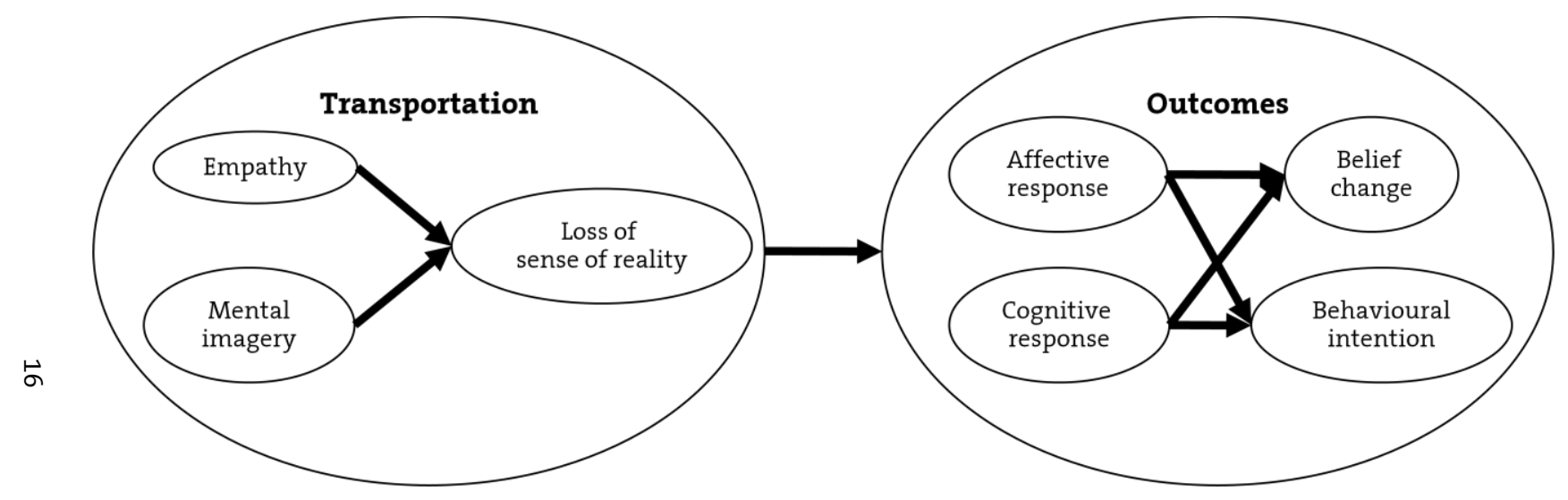

Figure 2-1

Conceptual model. 


\section{Outcomes}

\section{Affective response}

Narratives often elicit feelings (Escalas, Moore, \& Britton, 2004), which are also known as affective responses (Schwarz, 1990). To assess readers' agreement with feelings about a story, several studies directly measure their affective responses (e.g., Chang, 2009; Escalas, et al., 2004; LaMarre \& Landreville, 2009; Landreville \& LaMarre, 2010; Wang \& Calder, 2006). Furthermore, the generation of affective reactions can be influenced by transportation (e.g., LaMarre \& Landreville, 2009), such that high transportation causes readers to perceive the story as more realistic and thus express more emotional responses (LaMarre \& Landreville, 2009; Vaughn, 2010). Escalas (2004a) confirms that when a narrative evokes more transportation, readers generate more affect; Chang (2009) offers similar results. However, using a thought-listing procedure, West, Huber, and Min (2004) reveal that more transporting stories can generate fewer affective thoughts than less transporting ones.

\section{Cognitive response}

Readers tend to generate negative cognitive responses when confronted with a claim that runs counter their own beliefs (Moyer-Gusé \& Nabi, 2010), yet according to Green and Brock (2000, p. 702), transportation "may reduce negative cognitive responding." Slater and Rouner (2002, p. 180) offer empirical support for this claim and note that transported people often do not generate counterarguments "even if the persuasive subtext is inconsistent with prior attitudes, beliefs, or values." 
However, their studies never empirically observe or manipulate transportation in narrative worlds.

Dal Cin (2009) finds a relationship between transportation and positive cognitive responses; in a study of a message advocating smoking bans, she reports significant positive correlations between transportation and antismoking thoughts. Chang (2009) also finds that narrative magazine articles generate relatively more positive cognitive responses than do fact-based articles.

\section{Belief change}

Transportation can influence readers' beliefs (e.g., Braverman, 2008; Green \& Brock, 2000), because the more transported they are into a narrative, the more they disconnect from existing knowledge or beliefs (Green, 2004; Green \& Brock, 2000). Transported readers thus perceive story events as truthful (Green \& Brock, 2002), even if they know the narrative is fictional (Konijn, van der Molen, \& van Nes, 2009; Marsh \& Fazio, 2006). This perception of realism then prompts readers to engage in story-consistent thinking, which affects their beliefs (Escalas, 2004a, 2007; Mazzocco, Green, \& Brock, 2007; Wang \& Calder, 2006) and causes them to shift to become more convergent with the story's persuasive subtext (Appel \& Richter, 2007). However, most beliefs remain persistent with their position prior to the change (Cook \& Flay, 1978), and Tal-Or and colleagues (2004) demonstrate that narratives are persuasive only if readers imagine alternative outcomes to their own behaviour. Without this effort, beliefs that emerge because they have been transported and suspended their disbelief may disappear soon after they finish the story, such that readers retain their prior beliefs. 


\section{Behavioural intention}

When they are transported, readers tend to be more willing to perform an action (e.g., Dal Cin, Gibson, Zanna, Shumate, \& Fong, 2007; Dunlop, Wakefield, \& Kashima, 2008a; Escalas, 2004b; Escalas \& Luce, 2004; Schlosser, 2003). For example, $\mathrm{Dal}$ Cin and colleagues (2007) reveal that viewers transported by a movie in which the star smokes are more willing to smoke after they experience a high compared with a low level of transportation. Similarly, Schlosser (2003, Study 4) shows that consumers exhibit increased purchase intentions when they experience high transportation into the website of a digital camera manufacturer.

In summary, we anticipate that ceteris paribus, the transportation effect functions as follows:

$\mathbf{H}_{\mathbf{1}}$ : Transportation leads to (a) affective responses; (b) positive cognitive responses; (c) belief changes; and (d) behavioural intentions.

Using a meta-analytic model, we attempt to decipher differential effects that stem solely from measurement variance but remain sensitive to findings that imply different directions across studies. In other words, we seek meaningful relationships between transportation and affective and cognitive responses, belief changes, and behavioural intentions, about which prior studies concur.

In line with the postulates of transportation theory (Green, 2008; Green \& Brock, 2002, pp. 316317), our meta-analytic model comprises the reader, story, and medium attributes as three moderators of the effect of transportation on the outcomes of 
affective and cognitive responses, belief changes, and behavioural intentions. Furthermore, we acknowledge two ways to induce transportation (Green \& Brock, 2002): (1) empathy with the story characters or (2) mental imagery of the narrative events. The key difference between these inducements should reflect how stories are constructed: Empathy implies a focus on the story characters, whereas imagery suggests that the narrative events are central. However, most prior research attempts simply to determine if transportation exerts persuasive effects (for a review, see Slater, 2002a), without establishing how these effects function or which components have the strongest effects (cf., Chapter 4). We therefore consider empathy-based versus imagery-based approaches as a fourth potential moderator of transportation. Finally, our meta-analytic model notes the size of prior studies and several methodological factors that could moderate the transportation effect (e.g., use of random assignments, research domain, provision of incentives, participant demographics), as we detail in Figure 2-2. 


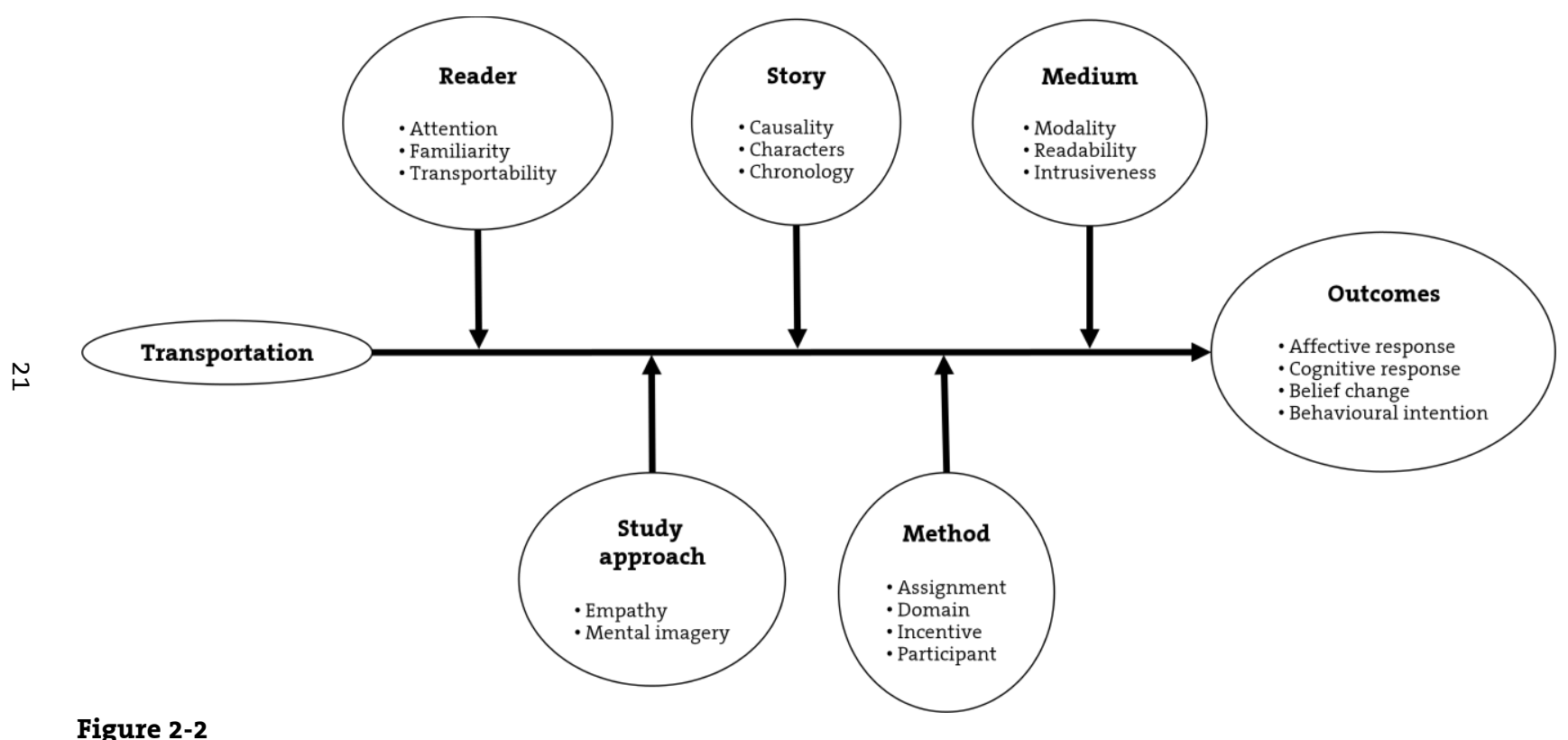

Meta-analytic model. 


\section{Reader moderators}

Transportation theory postulates that individual reader attributes, such as attention, familiarity, and transportability, influence the transportation effect (Green, 2008; Green \& Brock, 2002). Thus Gerrig's (1993) exposition of transportation highlights readers' attention to the story, and Greenwood (2008) reveals that cognitive interference correlates with lower levels of transportation. If readers' attention gets drawn away from the narrative, transportation and persuasion effects disappear (Vaughn, Hesse, Petkova, \& Trudeau, 2009). In contrast, a person with greater attention capacity can experience elevated transportation (Petrican, et al., 2008), such that when viewers focus on a movie, they are more likely to experience transportation (Polichak \& Gerrig, 2002), as are readers who enjoy reading and thus feel motivated to pay extra attention to the story (Green \& Brock, 2000).

Readers who are familiar with a story topic similarly may be more motivated to allow themselves to be transported into the narrative, whether due to their intrinsic interest or because they find it easier to imagine the story events (Slater, Rouner, \& Long, 2006). Familiarity thus facilitates the transportation effect by making story processing easier, such that people choose their preferred genre or story repeatedly. Susan Morgan, Movius, and Cody (2009) reveal for example that people who have donated organs experience more transportation into organ donation storylines than do non-donors, even when participants self-selected a show from among their own favourite genres. Green (2004) presents a story of a gay man visiting a college fraternity and finds that participants' closeness to someone who is gay and knowledge of 
Greek life in U.S. colleges predict their higher transportation. However, even readers without any gay friends or family indicate some transportation, which suggests familiarity is not an absolute prerequisite for transportation.

The third reader attribute, transportability, refers to a chronic propensity to be transported, which functions independently of any particular story (Dal Cin, Zanna, \& Fong, 2004). Transportability correlates with empathic ability (Davis, 1983) and image-producing capacity (Betts, 1909; Sheehan, 1967), and it induces transportation effects in reading experiences (Mazzocco, Green, Sasota, \& Jones, 2010), beyond just liking or habitually reading a genre (Bilandzic \& Busselle, 2008). In the antismoking advocacy context we described previously, transportability determines why people give in to smoking behaviours they observe in a story (Dal Cin, et al., 2007), and a high level of transportability enables both smokers and former smokers to recall narrative antismoking messages better than people marked by lower transportability (Dunlop, et al., 2008b). However, these latter two studies employ different transportability scales to capture this chronic propensity.

In summary, the effect of transportation should vary depending on readers' attention, familiarity, and transportability:

$\mathbf{H}_{2}$ : $\quad$ As readers (a) pay more attention to a story; (b) are more familiar with a topic; and (c) possess greater transportability, their propensity for transportation increases, thus increasing the effect of transportation on its outcomes. 


\section{Story moderators}

In their review of story elements, Brewer and Lichtenstein (1982) highlight three critical story attributes: causality, characters, and chronology. With regard to causality, Gergen and Gergen (1988) argue that any drama consists of a causal sequence of rising and falling events; more connections should lead to more drama. Accordingly, Green and Brock (2000) note that many canonical transporting stories feature tales of suffering. However, empirical evidence of a link between causality and the transportation effect is scarce. When Green and Brock (2000) compare alternate plots of identical stories, they find that readers report significantly less transportation into more trivial stories, but these authors never measure any other relevant outcomes or offer an a priori reason that causality should be necessary to influence beliefs and behavioural intentions. Only Adaval and Wyer Jr. (1998) lend some support to this relationship; in their study, causally connected travel stories prompt higher belief and intention ratings, compared with randomly connected information.

Similarity between readers and the focal character of a story also may help readers become transported, such that they adopt changed behavioural intentions (Moyer-Gusé, 2008; Sestir \& Green, 2010). However, violations of perceived realism cause readers to reject any similarity to the characters (Busselle \& Bilandzic, 2008). Empirically $\mathrm{Dal}$ Cin and colleagues (2007) establish that similarity is important, because similarity with a movie character who smokes increases viewers' intentions to smoke. Similarly, Greenwood (2007) determines that women's intention to engage in violent behaviours correlates with their perception of their similarity to heroic female characters. In a 
real-life setting, similarity with people portrayed in health communication narratives also can induce more nutritious eating habits among readers (Slater, Buller, Waters, Archibeque, \& LeBlanc, 2003).

Finally, chronology refers to the structure of the narrative and whether the story has a temporal dimension, with a beginning, middle, and end (Escalas, 2004b). Adherence to some narrative format is a minimal requirement of transportation (Green \& Brock, 2002), and a chronological structure often evokes suspense by concealing outcomes, whereas a lack of suspenseful adherence to the story format diminishes the opportunities for transportation (Green \& Brock, 2002). A linear chronological progression thus tends to create higher transportation, whereas a lack of chronological order induces only low transportation (Wang \& Calder, 2006). Furthermore, chronology and transportation both relate positively to the persuasive effect of narrative TV programmes (Nabi, Stitt, Halford, \& Finnerty, 2006).

Therefore, we speculate that causality, characters, and chronology moderate the transportation effect, as follows:

$\mathbf{H}_{3}$ : $\quad$ As stories have (a) a more causally connected chain of events; (b) characters more similar to readers; and (c) a more suspenseful chronological structure, their propensity for transportation increases, thus increasing the effect of transportation on its outcomes. 


\section{Medium moderators}

The moderating attributes of the medium might be positive or negative. For example, a richer modality and greater readability likely encourage the transportation effect, but intrusiveness might mitigate it. The same narrative can be delivered through various modalities (e.g., audio versus written), though most studies deal only with written messages (e.g., Green \& Brock, 2002). Braverman (2008) shows that transportation and persuasion depend on the modality, such that the effect of transportation becomes more pronounced when the narrative is delivered through a richer medium, because sound and images convey additional emotional information. Richer modalities also provide the imagery for recipients, such that they do not need to expend effort to imagine it. However, when Green and colleagues (2008) analyze the level of transportation for viewers who either did or did not experience specific imagery items, the results did not differ significantly.

Readability refers to the ease with which a person can process a narrative; it thus might depend on the font of written material (Vaughn, Petkova, Hesse, Trudeau, \& Ozses, 2007). Easy-to-read stories demand few cognitive resources from readers, but they also might not prompt cognitive responses to the narrative (Marsh \& Fazio, 2006). Furthermore, the greater readability of a narrative could increase processing fluency and transportation, but that influence in turn may inhibit negative cognitive responses (Vaughn, et al., 2007). In contrast with this reasoning, Petrova and Cialdini (2005) report positive effects of processing fluency on the use of images to change beliefs and behavioural intentions. 
Chang (2009) proposes that whether the narrative experience intrudes on the reader determines the transportation effect on that reader's affective and cognitive responses, beliefs, and intentions. Intrusive stories should lead to less transportation; nonintrusive narratives prompt greater transportation and persuasion. Wang and Calder (2009) test this theory by varying the position of a television commercial, such that the ad narrative interrupts the surrounding editorial content in one condition but not in another. Viewers expressed more transportation into the narrative when it was not intrusive.

On the basis of this previous research, we anticipate a stronger transportation effect when modality is richer and readability is greater but a weaker transportation effect when intrusiveness is greater.

$\mathbf{H}_{4}: \quad$ As media deliver (a) richer modalities and (b) greater readability, their propensity for transportation increases, thus increasing the effect of transportation on its outcomes.

However, when media (c) are more intrusive, their propensity for transportation decreases, thus decreasing the effect of transportation on its outcomes.

\section{Study approach moderators}

Various studies feature different transportation components, such as empathy with story characters or mental imagery, and these differences in turn may influence readers' responses to the narratives. First, narratives can evoke empathy with the story 
characters, such that readers try to understand deeply the experience of another, to know and feel the world in the same way such that the reader merges with the character's feelings (Escalas \& Stern, 2003). Slater and Rouner (2002) propose that empathy results in transportation, because readers vicariously experience the character's emotions, then express empathy for the character and become engrossed in the narrative (Green, 2004; Konijn, et al., 2009; Oatley, 2002). This situation prompts the readers to perceive events according to the bias of the character (Hoffner, Levine, \& Toohey, 2008). We find convincing evidence of this moderating influence; for example, differences in empathy influence readers' affective responses to the same narrative (Argo, Zhu, \& Dahl, 2008). In addition, Escalas, Moore, and Britton (2004) find that the heightened level of empathy elicited when consumers are transported into narrative commercials creates more positive cognitive evaluations. That is, empathy increases the likelihood that readers accept an underlying message and act accordingly (Deighton, Romer, \& McQueen, 1989; Mar, Oatley, \& Peterson, 2009).

Second, in Chapter 4, we conceptually and empirically distinguish empathy from mental imagery. Evocation of imagery appears vital to the transportation effect (Gerrig, 1993), such that in a mentally imagined setting, stories come to resemble real-life experiences (Green, 2006). In such a situation, the "constituents of the narrative, chiefly its evoked scenes, then take on new meaning as a result of their links with the experience of entering the narrative world" (Green \& Brock, 2002, p. 323). If people do not create mental imagery, they are less likely to experience transportation (Green, et al., 2004; Green, et al., 2008), yet creating mental imagery also draws down their cognitive resources 
and diminishes their capacity for cognitive responses (Lakshmanan \& Krishnan, 2009). Therefore, narratives should influence beliefs to the extent that those narratives provoke mental imagery and the accompanying transportation effect (Escalas, 2004a; Green \& Brock, 2000; Niederdeppe, Bu, Borah, Kindig, \& Robert, 2008). Readers also base their behavioural intentions on the ease with which they can imagine and follow narrative events (Petrova \& Cialdini, 2005; Schlosser, 2003). For example, Van den Hende and colleagues (2007) find that narratives about newly introduced concepts can evoke imagery and transportation, such that they help companies obtain valid customer feedback about high-tech innovations early in the product development process.

In line with Green and Brock's (2002) and Chapter 4's logic, we thus compare studies that focus on empathy with characters against studies that focus on mental imagery to determine how they might moderate the transportation effect.

$\mathrm{H}_{5}$ :

As studies focus more on (a) empathy and

(b) mental imagery, their propensity for transportation increases, thus increasing the effect of transportation on its outcomes.

Finally, we explore the role of four methodological moderators: whether the study used random assignments, the domain (i.e., communication, marketing, psychology, or technology), whether the study provided an incentive to participants, and the overall demographics of the participants (e.g., age, education). 


\section{Method}

\section{Sampling frame and search process}

To appear in our meta-analysis, a study must include transportation as a key variable. A vast array of experimental designs can qualify a study; furthermore, our review encompasses an inclusive linguistic range spanning Dutch, English, French, and German. We exclude studies in other languages, due to the difficulties of translation. We also limited our search to literature published after Green and Brock's (2000) empirical operationalization. Noting the potential upward bias of published studies (Bozarth \& Roberts, 1972), we deemed all relevant studies eligible, regardless of their publication form.

We employed various methods to support our literature search, as follows:

(1) Searches for the term "transportation" in the Business Source Premier, CINAHL, EconLit, ERIC, GreenFILE, International Bibliography of the Social Sciences, Library, Information Science \& Technology Abstracts, Philosopher's Index, PsyARTICLES, PsycINFO, Regional Business News, SocINDEX, Psychology and Behavioral Sciences Collection databases.

(2) A search of the Social Sciences Citation Index using Green and Brock's (2000) seminal article.

(3) Manual shelf searches of journals that publish empirical research on narratives, including the American Journal of Preventive Medicine, Communication Research, Discourse Processes, Health Communication, Human Communication Research, Journal of 
Advertising, Journal of Broadcasting \& Electronic Media, Journal of Communication, Journal of Consumer Psychology, Journal of Consumer Research, Journal of Experimental Social Psychology, Journal of Health Communication, Journal of Marketing Research, Journal of Nutrition Education and Behaviour, Journal of Personality and Social Psychology, Media Psychology, Narrative Inquiry, Poetics Today, and Sex Roles.

(4) E-mails sent to transportation scholars asking for published and unpublished works.

(5) Personal communications with Michael Slater, Sonya Dal Cin, Jennifer Escalas, Melanie Green, and Raymond Mar, the five most widely cited authors in the field of transportation, asking for their impressions of whether we were missing any important studies.

Our search produced 11 unpublished and 134 published articles (including book sections) related to transportation. ${ }^{2}$

\section{Coding of the meta-analysis variables}

To ensure the independence of the effect sizes, two expert psychology researchers classified the multitude of dependent variables reported in these studies into four categories: affective response, cognitive response, belief change (attitude and perception), and behavioural intention. These expert coders achieved acceptable agreement levels (Cohen's $\kappa=.74, \quad p<.001)$ and discussed any disagreements until they reached a common coding.

2 The complete list of articles is available on request from returnofthenarrative@gmail.com 
We provide some illustrative examples in Table 2-1. Furthermore, we confirmed that the effect sizes listed represented independent studies.

\section{Table 2-1}

Transportation, outcomes, and moderators operationalizations and representative papers.

\begin{tabular}{ll}
\hline Variable & $\begin{array}{l}\text { Operationalization } \\
\text { (representative papers) }\end{array}$ \\
\hline Transportation & Transportation scale (e.g., Chang, \\
& 2009; Green, 2004; Green \& \\
& Brock, 2000; LaMarre \& \\
& Landreville, 2009; Wang \& \\
& Calder, 2009); \\
& Experiential immersion scale \\
& (Bracken, 2006; Chang, 2008). \\
Outcomes & Thought-listing technique (e.g., \\
& Argo, et al., 2008; West, et al., \\
& 2004); \\
& Various scales (e.g., upbeat and \\
& warm, Escalas, et al., 2004; \\
& disgust and guilt, LaMarre \& \\
& Landreville, 2009). \\
& Thought-listing technique (e.g., \\
& Chang, 2009; Escalas, 2007; \\
& Green \& Brock, 2000, Study 1; \\
& Shrum, Burroughs, \& \\
& Rindfleisch, 2005; van Laer, \\
& 2005); \\
& "Pinochio" or false note-circling \\
& technique (e.g., Green \& Brock, \\
& 2000, Study 2 and 4). \\
\hline
\end{tabular}




\section{Table 2-1 - Continued}

Transportation, outcomes, and moderators operationalizations and representative papers.

\begin{tabular}{|c|c|c|}
\hline Variable & & $\begin{array}{l}\text { Operationalization } \\
\text { (representative papers) }\end{array}$ \\
\hline \multirow{5}{*}{\multicolumn{2}{|c|}{$\begin{array}{l}\text { Belief } \\
\text { change }\end{array}$}} & $\begin{array}{l}\text { Story-relevant true statements (e.g., } \\
\text { doctors work hard, S. E. Morgan, et } \\
\text { al., 2009); }\end{array}$ \\
\hline & & $\begin{array}{l}\text { Story-relevant false statements (e.g., } \\
\text { people can recover from brain death, } \\
\text { S. E. Morgan, et al., 2009); }\end{array}$ \\
\hline & & $\begin{array}{l}\text { Real-world belief scales (e.g., justice, } \\
\text { Green \& Brock, 2000; materialism, } \\
\text { Shrum, 2009); }\end{array}$ \\
\hline & & $\begin{array}{l}\text { Attitudes toward the story (topic) } \\
\text { scales (e.g., digital cameras, } \\
\text { Schlosser, 2003; bottled water, Wang } \\
\text { \& Calder, 2006); }\end{array}$ \\
\hline & & $\begin{array}{l}\text { Story-relevant perception scales (e.g., } \\
\text { credibility and realism, Bracken, } \\
\text { 2006; interestingness and } \\
\text { understandability, Braverman, 2008; } \\
\text { vividness, Chang, 2008; learning, S. E. } \\
\text { Morgan, et al., 2009; } \\
\text { informativeness, van den Hende, et } \\
\text { al., 2007). }\end{array}$ \\
\hline & $\begin{array}{l}\text { Behavioural } \\
\text { intention }\end{array}$ & $\begin{array}{l}\text { Various scales (e.g., drinking } \\
\text { moderation, Braverman, 2008; } \\
\text { political discussion, Landreville \& } \\
\text { LaMarre, 2010; organ donation, S. E. } \\
\text { Morgan, et al., 2009; purchase, } \\
\text { Schlosser, 2003; choice, West, et al., } \\
\text { 2004). }\end{array}$ \\
\hline
\end{tabular}


Table 2-1 - Continued

Transportation, outcomes, and moderators operationalizations and representative papers.

\begin{tabular}{|c|c|c|}
\hline Variable & & $\begin{array}{l}\text { Operationalization } \\
\text { (representative papers) }\end{array}$ \\
\hline \multirow[t]{6}{*}{ Reader } & Attention & $\begin{array}{l}\text { Instructions about how to read } \\
\text { the story, either inhibiting (e.g., } \\
\text { Escalas, 2007; Green, 2004; Green } \\
\text { \& Brock, 2000) or encouraging } \\
\text { (e.g., Schlosser, 2003, Study 4; } \\
\text { Vaughn, et al., 2007, Study 1) the } \\
\text { amount of attention readers pay } \\
\text { to the story. }\end{array}$ \\
\hline & \multirow[t]{2}{*}{ Familiarity } & $\begin{array}{l}\text { Familiarity with the story topic } \\
\text { test (e.g., relevant knowledge } \\
\text { about Greek life in American } \\
\text { colleges, Green, 2004); }\end{array}$ \\
\hline & & $\begin{array}{l}\text { Fit between story topic and } \\
\text { reader characteristics (e.g., having } \\
\text { read the book before seeing the } \\
\text { movie, Green, et al., 2008; being } \\
\text { an organ donor, S. E. Morgan, et } \\
\text { al., 2009). }\end{array}$ \\
\hline & \multirow[t]{3}{*}{ Transportability } & $\begin{array}{l}\text { Transportability scale (e.g., Dal } \\
\text { Cin, 2005; Dal Cin, et al., 2004); }\end{array}$ \\
\hline & & $\begin{array}{l}\text { Affect intensity scale (e.g., } \\
\text { Escalas, et al., 2004); }\end{array}$ \\
\hline & & $\begin{array}{l}\text { Empathic ability scale (e.g., Mar, } \\
\text { Oatley, Hirsh, dela Paz, \& } \\
\text { Peterson, 2006). }\end{array}$ \\
\hline
\end{tabular}




\section{Table 2-1 - Continued}

Transportation, outcomes, and moderators operationalizations and representative papers.

\begin{tabular}{|c|c|c|}
\hline Variable & & $\begin{array}{l}\text { Operationalization } \\
\text { (representative papers) }\end{array}$ \\
\hline \multirow[t]{5}{*}{ Story } & Causality & $\begin{array}{l}\text { Comparison between a dramatic and } \\
\text { trivial story (e.g., Green \& Brock, } \\
\text { 2000); }\end{array}$ \\
\hline & & $\begin{array}{l}\text { Comparison between a causal chain } \\
\text { and a random list of events (e.g., } \\
\text { Adaval \& Wyer Jr., 1998). }\end{array}$ \\
\hline & Characters & $\begin{array}{l}\text { Similarity between main character } \\
\text { and reader demographics (e.g., sexual } \\
\text { orientation, Green, 2004; gender, } \\
\text { Slater \& Rouner, 2002). }\end{array}$ \\
\hline & Chronology & $\begin{array}{l}\text { Story development, either following } \\
\text { or disrupting the linear chronology of } \\
\text { the events chain (Escalas, 2004b; e.g., } \\
\text { Escalas \& Stern, 2003; Vaughn, et al., } \\
\text { 2007, Pilot Study; Wang \& Calder, } \\
\text { 2006, Study 3); }\end{array}$ \\
\hline & & $\begin{array}{l}\text { Comparison between an analytical } \\
\text { and a narrative format (e.g., } \\
\text { Braverman, 2008; Chang, 2008; } \\
\text { Escalas, 2004a, 2007; van Laer, 2005). }\end{array}$ \\
\hline
\end{tabular}


Table 2-1 - Continued

Transportation, outcomes, and moderators operationalizations and representative papers.

\begin{tabular}{|c|c|c|}
\hline Variable & & $\begin{array}{l}\text { Operationalization } \\
\text { (representative papers) }\end{array}$ \\
\hline \multirow[t]{6}{*}{ Medium } & Modality & $\begin{array}{l}\text { Variety of channels (e.g., HDTV } \\
\text { versus NTSC, Bracken, 2006; written } \\
\text { versus spoken, Braverman, 2008; } \\
\text { written versus filmed, Green, et al., } \\
\text { 2008; drawn, photographed, or } \\
\text { animated, van den Hende, et al., } \\
\text { 2007). }\end{array}$ \\
\hline & \multirow[t]{3}{*}{ Readability } & $\begin{array}{l}\text { Field of view (e.g., ranging from } 53.1 \\
\text { to } 14.8 \text { degrees, Bracken, 2006); }\end{array}$ \\
\hline & & $\begin{array}{l}\text { Font clarity (e.g., white versus gray, } \\
\text { Vaughn, et al., 2007); }\end{array}$ \\
\hline & & $\begin{array}{l}\text { Flesch readability index (e.g., 6th- } \\
\text { grade versus high school reading } \\
\text { levels, Marsh \& Fazio, 2006). }\end{array}$ \\
\hline & \multirow[t]{2}{*}{ Intrusiveness } & $\begin{array}{l}\text { Fit between the story topic and the } \\
\text { surrounding editorial content (e.g., } \\
\text { Chang, 2009; Wang \& Calder, 2009); }\end{array}$ \\
\hline & & $\begin{array}{l}\text { Fit between the story time span and } \\
\text { real time (e.g., Vaughn, Petkova, } \\
\text { Hesse, Trudeau, \& McCaffrey, 2006). }\end{array}$ \\
\hline
\end{tabular}




\section{Table 2-1 - Continued}

Transportation, outcomes, and moderators operationalizations and representative papers.

\begin{tabular}{|c|c|c|}
\hline Variable & & $\begin{array}{l}\text { Operationalization } \\
\text { (representative papers) }\end{array}$ \\
\hline \multirow[t]{7}{*}{$\begin{array}{l}\text { Study } \\
\text { approach }\end{array}$} & \multirow[t]{3}{*}{ Empathy } & $\begin{array}{l}\text { Instructions about how to read the } \\
\text { story, encouraging readers to } \\
\text { empathize with the story characters } \\
\text { (e.g., Green \& Brock, 2000); }\end{array}$ \\
\hline & & $\begin{array}{l}\text { Telling the story from different point } \\
\text { of views (e.g., either interaction } \\
\text { partner, de Graaf, Sanders, Beentjes, \& } \\
\text { Hoeken, 2007; first- versus second- } \\
\text { person, West, et al., 2004); }\end{array}$ \\
\hline & & $\begin{array}{l}\text { Various scales (e.g., emotional } \\
\text { involvement, Bracken, 2006; character } \\
\text { liking, Chang, 2009). }\end{array}$ \\
\hline & \multirow[t]{4}{*}{$\begin{array}{l}\text { Mental } \\
\text { imagery }\end{array}$} & $\begin{array}{l}\text { Instructions about how to read the } \\
\text { story, encouraging readers to imagine } \\
\text { the narrative events (e.g., Schlosser, } \\
\text { 2003); }\end{array}$ \\
\hline & & $\begin{array}{l}\text { Telling the story using vivid } \\
\text { descriptions of narrative events (e.g., } \\
\text { Escalas, 2004a); }\end{array}$ \\
\hline & & $\begin{array}{l}\text { Thought-listing technique (e.g., } \\
\text { Escalas, 2007; West, et al., 2004); }\end{array}$ \\
\hline & & $\begin{array}{l}\text { Various scales (e.g., vividness, de } \\
\text { Graaf, et al., 2007; enjoyable } \\
\text { imagination, Green, et al., 2008, Study } \\
\text { 1). }\end{array}$ \\
\hline
\end{tabular}

We analyzed a total of 287 effect sizes. The coding mandated that a positive effect size indicated a greater transportation effect, whereas a negative effect size indicated a smaller transportation effect. Differences in the number of positive versus negative cognitive responses revealed effects on cognitive response. For example, in a comparison of a narrative and an analytical 
format, we determined a positive transportation effect on affective responses (Escalas, et al., 2004), beliefs, and behavioural intentions (Braverman, 2008; Escalas, 2004a) but a negative effect on cognitive responses (Chang, 2009). The positive effects indicated that the means for the first three outcomes were greater in the narrative format condition than in the analytical format condition; however, the dominant cognitive response was greater in the analytical format condition than in the narrative format condition.

\section{Effect size computation}

The effect size statistic contrasts groups according to their mean scores on transportation and its outcomes. The Pearson correlation provides our effect size indicator. We used Hullett and Levine's (2003) method to calculate a simple average effect size by converting $F$ values from the analyses of variance into effect size estimates. We corrected these estimates for differences in the reliability of the variables involved. Our formula for correcting a correlation coefficient $\left(r_{u}\right)$ between variables $\mathrm{x}$ and $\mathrm{y}$ is

$$
r_{\mathrm{u}}=\frac{\sqrt{r_{\mathrm{xy}}}}{\sqrt{r_{\mathrm{xx}}} \sqrt{r_{\mathrm{yy}}}}
$$

where $r_{x x}$ and $r_{y y}$ represent the measurement reliabilities of $\mathrm{x}$ and $\mathrm{y}$, respectively (Hunter \& Schmidt, 2004). We also adapted this formula by setting $r_{\mathrm{xy}}$ equal to $r_{\text {contrast }}$ and $r_{\mathrm{xx}}$ equal to 1 . Fortunately, most studies reported coefficient reliability values (e.g., Cronbach's $\alpha$, Cohen's $\kappa$ ), which we could use to estimate the dependent measures of $r_{\mathrm{yy}}$. 
To test the effect of transportation on each outcome and the moderating effects of the relevant variables, we calculated not only the sampleweighted reliability-adjusted $r$ but also the 95\% confidence interval and the conservative random effect $z$ (Hunter \& Schmidt, 2004). To determine the presence of heterogeneity, we used the $Q$ statistic, and to determine the extent of such heterogeneity, we adopted the $I^{2}$ index (Huedo-Medina, SánchezMeca, Marín-Martínez, \& Botella, 2006). In addition, we determined the file drawer $N$, which indicates the number of studies with a zero effect size that would be required to reduce the mean effect size to a probability level of $\alpha=.05$ (Rosenthal, 1991). In support of our analysis, we relied on the Comprehensive Meta-Analysis software program (Borenstein, Hedges, Higgins, \& Rothstein, 2005).

\section{Results}

\section{Homogeneity and sampling bias check}

We first examine the main effect of transportation on the outcome variables, as we summarize in Table 2-2. 
Table 2-2

Descriptive statistics.

\begin{tabular}{|c|c|c|c|c|c|c|c|c|c|}
\hline \multirow[t]{2}{*}{ Outcomes } & \multirow[t]{2}{*}{$\begin{array}{l}\text { Total } \\
N^{\mathrm{a}}\end{array}$} & \multirow[t]{2}{*}{$\begin{array}{l}\text { Number } \\
\text { of studies }\end{array}$} & \multirow[t]{2}{*}{$r$} & \multirow[t]{2}{*}{$\begin{array}{l}\text { Reliability- } \\
\text { adjusted } r\end{array}$} & \multirow[t]{2}{*}{$\begin{array}{l}\text { Sample- } \\
\text { weighted } \\
\text { reliability- } \\
\text { adjusted r }\end{array}$} & \multicolumn{2}{|c|}{$\begin{array}{l}\text { Confidence } \\
\text { interval }\end{array}$} & \multirow{2}{*}{$\begin{array}{l}\text { Test } \\
\text { of } \\
\text { null }\end{array}$} & \multirow{2}{*}{$\begin{array}{l}\text { Homogeneity } \\
\text { test } \\
\text { Q }\end{array}$} \\
\hline & & & & & & $\begin{array}{l}\text { Lower } \\
\text { limit }\end{array}$ & $\begin{array}{l}\text { Upper } \\
\text { limit }\end{array}$ & & \\
\hline $\begin{array}{l}\text { Affective } \\
\text { response }\end{array}$ & 10,383 & 72 & .13 & .11 & .11 & .06 & .16 & $4.43^{* * *}$ & $498.35^{* * *}$ \\
\hline $\begin{array}{l}\text { Cognitive } \\
\text { response }\end{array}$ & 12,609 & 33 & .07 & .07 & .07 & .00 & .14 & $1.99^{*}$ & $227.71^{*}$ \\
\hline Belief change & 56,446 & 152 & .09 & .11 & .11 & .08 & .14 & $6.91^{* * *}$ & $2972.95^{* * *}$ \\
\hline $\begin{array}{l}\text { Behavioural } \\
\text { intention }\end{array}$ & 32,579 & 30 & .23 & .22 & .22 & .14 & .29 & $5.67^{* * *}$ & $815.82^{* * *}$ \\
\hline
\end{tabular}

${ }^{*} p<.05{ }^{* * *} p<.001$.

${ }^{\mathrm{a}}$ Total number of participants in the original studies. 
Focusing on the reliability-adjusted, sampleweighted $r$ effect size, we find that consistent with $\mathrm{H}_{1 a}, \mathrm{H}_{1 \mathrm{c}}$, and $\mathrm{H}_{1 \mathrm{~d}}$, transportation has significant, positive, medium-sized effects on affective responses $\left(r=.13, Q_{(71)}=498.35, p<.001, I^{2}=85.75\right.$, file drawer $\quad N=3,127)$, belief changes $(r=.09$, $Q_{(151)}=2972.95, p<.001, I^{2}=94.92$, file drawer $\left.N=8,001\right)$, and behavioural intentions $\left(r=.23, Q_{(29)}=815.82\right.$, $p<.001, I^{2}=96.45$, file drawer $\left.N=3,304\right)$. The file drawer results in particular demonstrate that the positive effect of transportation is robust and probably not a reflection of publication bias; it would require nearly 8,000 null effects to reduce the statistical significance of the effect of transportation on belief changes to .05 at the one-tailed level. The main effect of transportation on cognitive responses is small but positive $\left(r=.07, Q_{(32)}=227.71, p<.05\right.$, $I^{2}=85.95$, file drawer $N=274$ ), in support of $\mathrm{H}_{1 \mathrm{~b}}$. The $Q$ statistic is significant for all four relationships, such that the effect sizes are heterogeneous, and it is beneficial to assess the factors that moderate these relationships.

\section{Moderator hypotheses tests}

We consider each moderator separately. Because we determine positive main effects for all outcomes, we can merge affective and cognitive responses, belief changes, and behavioural intentions into an overall transportation effect for this analysis. We report the number of effects, the average sample-weighted reliability-adjusted $r$ for each level of each moderator, and any differences in the random effects across the levels of the moderator in Table 2-3. Missing data cause the number of observations used to test each moderator to vary. 
Table 2-3

Transportation effect moderated by reader, story, medium, and study approach.

\begin{tabular}{|c|c|c|c|c|c|c|c|}
\hline \multirow[t]{2}{*}{ Moderators } & & \multirow{2}{*}{$\begin{array}{l}\text { Number } \\
\text { of } \\
\text { studies }\end{array}$} & \multirow{2}{*}{$\begin{array}{l}\text { Sample- } \\
\text { weighted } \\
\text { reliability- } \\
\text { adjusted } r\end{array}$} & \multicolumn{2}{|c|}{ Confidence interval } & \multirow{2}{*}{$\begin{array}{l}\text { Test } \\
\text { of } \\
\text { null }\end{array}$} & \multirow{2}{*}{$\begin{array}{l}\text { Homogeneity } \\
\text { test } \\
\text { Q }\end{array}$} \\
\hline & & & & $\begin{array}{l}\text { Lower } \\
\text { limit }\end{array}$ & $\begin{array}{l}\text { Upper } \\
\text { limit }\end{array}$ & & \\
\hline \multirow[t]{2}{*}{ Reader } & Attention & 39 & .06 & -.01 & .14 & 1.62 & $1725.78^{* * *}$ \\
\hline & Transportability & 6 & .27 & .11 & .42 & $3.29^{* *}$ & \\
\hline \multirow[t]{3}{*}{ Story } & Causality & 2 & .41 & .16 & .60 & $3.16^{* *}$ & $1594.84^{* * *}$ \\
\hline & Characters & 12 & .02 & -.12 & .17 & .32 & \\
\hline & Chronology & 150 & .10 & .08 & .13 & $8.10^{* * *}$ & \\
\hline Medium & Intrusiveness & 10 & -.09 & -.17 & -.01 & $-2.25^{*}$ & \\
\hline
\end{tabular}


Table 2-3 - Continued

Transportation effect moderated by reader, story, medium, and study approach.

\begin{tabular}{lllllll}
\hline Moderators & & $\begin{array}{l}\text { Number of } \\
\text { studies }\end{array}$ & $\begin{array}{l}\text { Sample- } \\
\text { weighted } \\
\text { reliability- } \\
\text { adjusted } r\end{array}$ & Confidence interval & $\begin{array}{l}\text { Test } \\
\text { of } \\
\text { null }\end{array}$ & $\begin{array}{l}\text { Homogeneity } \\
\text { test }\end{array}$ \\
\hline
\end{tabular}

${ }^{*} p<.05 ;{ }^{* *} p<.01 ;{ }^{* * *} p<.001$. 
When readers experience transportation, their own characteristics influence the salience of the experience; that is, reader attributes strongly moderate the transportation effect $\left(Q_{(84)}=1725.78\right.$, $\left.p<.001, \quad I^{2}=95.13\right)$. The effect sizes indicate specifically that the transportation effect differs depending on readers' transportability $(r=.27)$ and familiarity $(r=.22)$, in support of $\mathrm{H}_{2 b}$ and $\mathrm{H}_{2 c}$. However, we find no support for $\mathrm{H}_{2 \mathrm{a}}$ because no significant moderation emerges for attention.

Previous research attests that the transportation effect stems from dramatic chains of causally connected events, similarity between characters and readers, and a chronological story structure. Our results support the overall prediction that the transportation effect varies for different story characteristics $\quad\left(Q_{(163)}=1594.84, \quad p<.001\right.$, $I^{2}=89.78$ ); this variation is especially strong for causality $(r=.41)$ and less so for chronology $(r=.10)$, but we find support for both $\mathrm{H}_{3 \mathrm{a}}$ and $\mathrm{H}_{3 \mathrm{c}}$. We find no significant differences as a function of character similarity, so we must reject $\mathrm{H}_{3 \mathrm{~b}}$.

Regarding the various media available, we argue that medium attributes affect the transportation effect through modality, readability, and intrusiveness. The pattern of results offers support for $\mathrm{H}_{4 \mathrm{~b}}$ and $\mathrm{H}_{4 \mathrm{c}}$ : The transportation effect is stronger with greater readability $(r=.09)$ and less intrusive media $\left(r=-.09 ; \quad Q_{(50)}=1205.88, \quad p<.001\right.$, $I^{2}=95.85$ ). However, modality richness does not enhance the transportation effect, so we cannot confirm $\mathrm{H}_{4 a}$.

Furthermore, we expected the transportation effect to be stronger when studies focus on either empathy or mental imagery. In support of $\mathrm{H}_{5}$, we find a stronger transportation effect for both components $\left(Q_{(252)}=3840.08, \quad p<.001, \quad I^{2}=93.44\right)$. 
Specifically, the results indicate a stronger transportation effect for empathy $(r=.11)$ than for mental imagery $(r=.09)$.

Next, we consider the effects of four methodological variables as moderators of the transportation effect (see Table 2-4). We find a stronger transportation effect for studies that explicitly mention the use of random assignments $\left(r_{\text {Random }}=.13, \quad r_{\text {Non-random }}=.05, \quad Q_{(176)}=3526.04, \quad p<.001\right.$, $\left.I^{2}=95.01\right)$. The domain of the study also creates differences $\left(Q_{(239)}=3770.17, p<.001, I^{2}=93.66\right)$, such that studies in the communication $(r=.12)$ and marketing $(r=.10)$ domains reveal consistently high effect sizes. Moreover, if a study offers participants an incentive-whether in the form of money, snacks, or course credit-the transportation effect is enhanced $\left(r_{\text {Incentive }}=.14, r_{\text {No incentive }}=.06, Q_{(262)}=4473.14\right.$, $\left.p<.001, \quad I^{2}=94.14\right)$. There is also a stronger transportation effect for participants with a university education ( $\left.r_{\text {Graduate }}=.11 \quad r_{\text {Undergraduate }}=.12\right)$ compared with those without it $(r=.06$, $\left.Q_{(281)}=4507.75, p<.001, I^{2}=93.77\right)$. We find stronger effects in North American $\left(r_{\text {Canadian }}=.20, r_{\text {American }}=.11\right)$ and Dutch $\left(r=.11, Q_{(286)}=4568.01, p<.001, I^{2}=93.74\right)$ cultures too. Finally, our meta-regression analyses pertaining to the moderators of age and gender (i.e., proportion of women) indicate a stronger transportation effect for younger readers $(r=-.00$, $\left.Q_{(1)}=7.40, \quad p<.01, \quad \tau^{2}=.03\right)$ and for men $(r=-.18$, $\left.Q_{(1)}=152.26, p<.001, \tau^{2}=.03\right)$. 


\section{Table 2-4}

Transportation effect moderated by assignment, domain, incentive, and participant age, education, gender, and nationality.

\begin{tabular}{|c|c|c|c|c|c|c|c|}
\hline \multirow[t]{2}{*}{ Moderators } & & \multirow[t]{2}{*}{$\begin{array}{l}\text { Number of } \\
\text { studies }\end{array}$} & \multirow{2}{*}{$\begin{array}{l}\text { Sample- } \\
\text { weighted } \\
\text { reliability- } \\
\text { adjusted } r\end{array}$} & \multicolumn{2}{|c|}{$\begin{array}{l}\text { Confidence } \\
\text { interval }\end{array}$} & \multirow{2}{*}{$\begin{array}{l}\text { Test } \\
\text { of } \\
\text { null } \\
z\end{array}$} & \multirow{2}{*}{$\begin{array}{l}\text { Homogeneity } \\
\text { test } \\
\text { Q }\end{array}$} \\
\hline & & & & $\begin{array}{l}\text { Lower } \\
\text { limit }\end{array}$ & $\begin{array}{l}\text { Upper } \\
\text { limit }\end{array}$ & & \\
\hline Assignment & Random & 144 & 0.13 & 0.07 & 0.19 & $4.31^{* * *}$ & $3526.04^{* * *}$ \\
\hline \multirow[t]{4}{*}{ Domain } & Communication & 98 & 0.12 & 0.08 & 0.15 & $6.33^{* * *}$ & $3770.17^{* * *}$ \\
\hline & Marketing & 92 & 0.10 & 0.04 & 0.16 & $3.27^{* *}$ & \\
\hline & Psychology & 41 & 0.04 & -0.01 & 0.09 & 1.52 & \\
\hline & Technology & 9 & 0.18 & -0.07 & 0.41 & 1.41 & \\
\hline
\end{tabular}




\section{Table 2-4 - Continued}

Transportation effect moderated by assignment, domain, incentive, and participant age, education, gender, and nationality.

\begin{tabular}{|c|c|c|c|c|c|c|c|c|}
\hline \multicolumn{3}{|l|}{ Moderators } & \multirow[t]{2}{*}{$\begin{array}{l}\text { Number } \\
\text { of } \\
\text { studies }\end{array}$} & \multirow{2}{*}{$\begin{array}{l}\text { Sample- } \\
\text { weighted } \\
\text { reliability- } \\
\text { adjusted r }\end{array}$} & \multicolumn{2}{|c|}{$\begin{array}{l}\text { Confidence } \\
\text { interval }\end{array}$} & \multirow{2}{*}{$\begin{array}{l}\text { Test } \\
\text { of } \\
\text { null } \\
z\end{array}$} & \multirow{2}{*}{$\begin{array}{l}\text { Homogeneity } \\
\text { test } \\
\underline{Q}\end{array}$} \\
\hline & & & & & $\begin{array}{l}\text { Lower } \\
\text { limit }\end{array}$ & $\begin{array}{l}\text { Upper } \\
\text { limit }\end{array}$ & & \\
\hline \multirow[t]{2}{*}{ Incentives } & Incentive & & 200 & 0.14 & 0.10 & 0.18 & $6.67^{* * *}$ & $4473.14^{* * *}$ \\
\hline & $\begin{array}{l}\text { No } \\
\text { incentive }\end{array}$ & & 63 & 0.06 & 0.02 & 0.10 & $2.99^{* *}$ & \\
\hline \multirow[t]{3}{*}{ Participant } & Education & Graduate & 33 & 0.11 & 0.06 & 0.15 & $4.64^{* * *}$ & $4507.75^{* * *}$ \\
\hline & & Undergraduate & 240 & 0.12 & 0.08 & 0.16 & $6.05^{* * *}$ & \\
\hline & & $\begin{array}{l}\text { Not university } \\
\text { educated }\end{array}$ & 9 & 0.06 & 0.05 & 0.07 & $10.00^{* * *}$ & \\
\hline
\end{tabular}




\section{Table 2-4 - Continued}

Transportation effect moderated by assignment, domain, incentive, and participant age, education, gender, and nationality.

\begin{tabular}{cllllllll}
\hline Participant & Nationality & American & 175 & 0.11 & 0.08 & 0.14 & $6.66^{* * *}$ & $4568.01^{* * *}$ \\
& & Australian & 3 & 0.14 & -0.15 & 0.41 & 0.82 & \\
& & Belgian & 4 & 0.06 & -0.23 & 0.35 & 0.42 & \\
& & British & 2 & 0.22 & -0.07 & 0.47 & 1.51 & \\
& & Canadian & 46 & 0.20 & 0.15 & 0.24 & $7.81^{* * *}$ & \\
& & Dutch & 40 & 0.11 & 0.05 & 0.18 & $3.25^{* *}$ & \\
& German & 5 & 0.01 & -0.28 & 0.31 & 0.08 & \\
& Taiwanese & 12 & 0.04 & -0.04 & 0.13 & 1.04 & \\
& & & 287 & -.00 & -.00 & -.00 & $-2.72^{* *}$ & $7.40^{* *}$ \\
& Age & & 287 & -.18 & -.21 & -.15 & $-12.34^{* * *}$ & $152.26^{* * *}$ \\
& Gender & & & & & & &
\end{tabular}

${ }^{* *} p<.01 ;{ }^{* * *} p<.001$. 


\section{Discussion}

The findings of our meta-analysis provide several insights regarding the collected research into the transportation effect. Primarily, the effects of transportation consistently are in the predicted direction, significant, and of medium range (i.e., from .11 for affective response and belief change to .22 for behavioural intention) for most of the dependent variables in Figure 2-2. In line with $\mathrm{Dal}$ Cin's (2009) suggestions, our results indicate that transportation leads to more positive cognitive responses, though this effect appears significantly smaller than the others. Perhaps a more complex pattern underlies the effect of transportation on cognitive responses. For example, Slater and Rouner (2002) suggest multiple predictors that could be positively associated with such responses (e.g., perceived narrative interest, quality).

Overall we can assign the moderators into three categories: narrative communication-related (i.e., reader, story, and medium), study approach-related (i.e., empathy versus mental imagery), and methodological (i.e., random assignments, domain, use of incentives, and subjects). All three narrative communication variables moderate the transportation effect, and as we predicted, greater reader familiarity and transportability and a good story chronology increase the transportation effect. Yet as a surprising finding, we note the large effect of causal connectedness. Empirical studies that focus on causality offer only minimal replications, yet this moderator demonstrates a consistent, significant difference. These results highlight the importance of a causally connected chain of events. It is also interesting to note that the significant 
effects of the medium, related to readability and intrusiveness, are of equal magnitude but in opposite directions. Thus, we might question if they represent different characteristics of a specific medium. Although the results support the theory that a propensity toward transportation depends on several attributes of narrative communication elements (Green \& Brock, 2002), we find that reader attention, story characters, and the medium's modality do not significantly moderate the transportation effect. Green (2004) offers an interpretative explanation that conceptualizes attention for the story as an integrative part of physiological awareness. Regarding the unexpected results pertaining to characters, we posit that perceived similarity might not translate directly to transportation. Green and Brock (2000) claim transportation refers to a general state, whereas character similarity is highly specific (Sestir \& Green, 2010). Finally, Green and colleagues (2008) assess the transportation effect of two media modalities and find that film or print media affect transportation similarly. However, when they include subscales, the modalities differ significantly for the mental imagery subscale, such that readers of the print version report greater transportation. These different effects according to different transportation scales indicate that the best measure is likely a composite scale.

Empathy creates a greater transportation effect than mental imagery, but because the difference in the sizes of these effects is small, we propose that transportation theory appears generalizable across both components. In this case, our results reinforce Green and Brock's (2000) finding that their ultimate scale, which combines the best version of each established subscale, is an effective measure for exploring transportation. 
Finally, greater transportation occurs when a study assigns participants randomly, focuses on a marketing or communication domain, provides incentives, and includes highly educated, culturally diverse, young, male participants. Participants who receive an incentive likely are more involved, which can increase the transportation effect. We also note though that most of the research in our metaanalysis uses student samples and random assignments, so we have far fewer studies that include non-university educated and non-randomly assigned groups. Although we report an age difference, the small size of this effect implies that it has little impact. The gender difference is greater; men report significantly greater transportation than women. Perhaps men have a greater need to idealize story characters, which increases their transportation (Denham, 2004). Finally, communication and marketing studies, especially those conducted in Canada and the United States, offer fruitful contexts, because in these domains, the effect of transportation is particularly strong. Thus, transportation theory should have particularly strong consumer behaviour implications in North America.

\section{Limitations}

Meta-analytic studies, like any other type of study, suffer certain limitations. We have attempted to explain heterogeneity in four main effects using five potential moderators, but the case can always be made that additional moderators account for heterogeneity. Furthermore, we did not examine how these variables moderate the influence of the transportation effect on affective and cognitive responses, belief changes, and behavioural 
intentions separately, because we had so few effects. We also could not examine the effect of transportation on actual behaviour because we lack sufficient data. Over time, as additional studies tackle these issues, it will be easier to determine such effects.

We could not code some potential moderators because we lacked sufficient information, including medium interactivity and the persistence of narrative persuasion effects. Our results imply a stronger transportation effect for readers with higher transportability, apparently because of its chronic influence. As Dal Cin (2005) points out, these constructs are not orthogonal.

This chapter underscores the robustness of the transportation effect and builds on previous research to refine our extant understanding of it. Transportation has a significant impact on each stage of narrative processing, from the mental processing invested to imagine the events to changes in readers' beliefs. Moreover, transportation effects appear moderated by key variables, such as the reader's familiarity with the topic and the story's causal connectedness. These findings have implications for not only persuasion research but communication practices overall-as demonstrated by the growing popularity of public narratives that dramatically influence the way people process and share information and make decisions. As members of society increasingly experience transportation by dealing with persuasive narratives, it also becomes increasingly important to understand the underlying process of narrative communication. 
We have identified some paths that research underlying this dissertation has trod and thus hope to enable scholars and practitioners alike to see the forest for the trees, rather than getting lost in transportation. 



\section{3 \\ A WALK IN CUSTOMERS' SHOES}

How narrative transportation affects ownership of integrity-violating blog posts

\section{Introduction}

As social media proliferate, such that markets get defined as conversations (Searls \& Weinberger, 2009), the impact of public opinion changes. Whereas once customer voices may have fallen on deaf ears, today people can easily share their disappointing consumption experience with a receptive, massive audience by communicating through the rapidly growing medium of weblogs (blogs) (Hennig-Thurau, et al., 2010). Millions of incensed bloggers thus accused Citigroup of distorting investment research reports to manipulate consumer investment decisions. Even more than competence failures or product recalls, online accounts of unethical employee behaviour erode consumer trust and dramatically influence the way they process and share information (Gartner Research, 2007; The Economist, 2006; Woodside, et al., 2008). Yet stories of unfair practices remain largely beyond the control of companies (Deighton \& Kornfeld, 2009). In the context of social media, statements from official spokespeople appear to be "cheap talk" (Farrell \& Rabin, 1996), whereas consumers might relate more with workers in the lower ranks (Gaines-Ross, 2010). Therefore, an 
increasing number of organizations, including Best Buy, British Telecom, Dell, Hewlett-Packard, Microsoft, SAP, TNT, Zappos, and even the U.S. Army, now encourage employees to respond personally to social media messages. In so doing, they must ensure that employees respond appropriately by putting themselves in the customer's shoes before issuing their responses to concerns voiced on blogs. A recent marketing decision maker survey (SAS, 2009) thus identifies a demand for mental triggers that can drive employees to adopt a mindset in which they assume ownership of events that prompt customer complaints in blogs and thereby offer a response that is more likely to restore customer trust.

Recent advances in narrative transportation research may address this demand. Transportation refers to an integrative melding of reader, story, and medium, focused on story characters and events (Chapter 2, p. 14). Mounting empirical evidence indicates that being engrossed in a narrative account of someone else's experience facilitates other-regarding beliefs and intentions (Escalas \& Stern, 2003; Polichak \& Gerrig, 2002). Thus far though, examinations of the persuasive influence of transportation focus primarily on entertainment and advertising (e.g., Escalas, 2007; Green \& Brock, 2002; Slater, 2002b). We instead propose that reading customer accounts of integrity violations on blogs might cause employees to be transported into the customers' perspective, causing them to feel a sense of ownership or obligation to deal with the problem at hand.

However, when employees confront reported violations of integrity, they instinctively tend to exhibit external, rather than self, attributions (Folkes, 1988; Folkes \& Kotsos, 1986). In addition, 
the influence of transportation is subject to considerable variation across readers, depending on their ability to empathize with others for example (Dal Cin, et al., 2004). Transportation also varies according to the relationship between prior knowledge about the personal circumstances of the protagonist and the severity of the events described (Green, 2004). We therefore take such variations into account when determining how companies might use narrative transportation as a vehicle for implementing an organization-wide sense of responsibility to take action in response to customer blogs.

Rather than relying solely on employees' general disposition to take a customer perspective and assume ownership of the task of restoring integrity, it may be more effective to induce a biased mindset that triggers transportation and key ownership dimensions, namely, acknowledgement of responsibility and intention to respond. Pennebaker, Mehl, and Niederhoffer (2003) show that others' perspectives can be induced momentarily and promote a desirable response, though the ability to put on another person's "mental shoes" is a sophisticated process that requires empathy. To develop an in-depth understanding, we explore the potential of priming empathy as a driver of narrative transportation, in relation to employees' ownership of integrity violations. This exploration reflects two main research objectives.

First, we posit that perspective taking can prime transportation, but we also need to attend to both cognitive and affective elements. Perspective taking is clearly a cognitive mindset that drives other-regarding behaviour (cf., Smeesters, Wheeler, \& Kay, 2009), but it also might be affect based 
(Lamm, Batson, \& Decety, 2007). Customer accounts of integrity violations on blog posts offer affectladen experiential stories, in which the customer is the protagonist (Delgadillo \& Escalas, 2004; Kozinets, de Valck, Wojnicki, \& Wilner, 2010). Reading a story from the protagonist's point of view enhances the probability that readers share this character's worldview. Yet for transportation to provoke a sense of responsibility and a willingness to respond, we posit that it is not sufficient to take another's perspective; the employee must also be mindful of the other's affective condition (Gerrig, 1993; Green \& Brock, 2002). Thus, triggering an empathic perspective may address both affective and cognitive elements of integrity violations. We extend current research by advancing this empathic perspective to enhance the effectiveness with which transportation encourages employees' ownership. Thus we empirically assess whether this perspective leads to a higher sense of responsibility and willingness to respond, prior to formulating an actual response to a blog post.

Second, we examine an important boundary condition of empathic perspectives. According to Dunlop, Wakefield, and Kashima (2008a), empathy gets experienced predominantly when people recognize or perceive a particular relationship between the narrator and the narrative events. Relevant prior knowledge about the narrator's personal circumstances makes it easier to find this connection. In the case of integrity violations reported in blog posts, a customer's vulnerability to the integrity violation may provide such relevant information. That is, empathy should be triggered when the employee relates the customer's vulnerability to the integrity violation, such that mental triggers of an empathic perspective could be strengthened or weakened by customer 
vulnerability. In a field experiment with employees of a large financial institution, we assess whether this line of reasoning fits with regard to the impact of financial vulnerability on transportation (for an overview, see Figure 3-1).

\section{Conceptual background}

\section{Integrity violation ownership}

We develop our conceptual background by elaborating first on the need to assume psychological ownership of an integrity violation to repair a relationship. An emerging theme, integrity violation refers to the situation that occurs when one party perceives that another party is no longer adhering to acceptable or necessary principles and standards in the relationship (Ferrin, Kim, Cooper, \& Dirks, 2007; Kim, Ferrin, Cooper, \& Dirks, 2004; R. C. Mayer \& Davis, 1999). For employees to restore customers from their perceptions of such violations, they must assume ownership of the integritydestroying events described in the customers' blog posts. Ownership of integrity violations appears to involve a four-dimensional structure: recognizing that the events destroy integrity, (2) determining the cause of the reduced integrity, (3) acknowledging where the responsibility for the consequences rests, and (4) being willing to respond to the integrity violation (Itoi, Ohbuchi, \& Fukuno, 1996; Lewicki \& Bunker, 1996). 


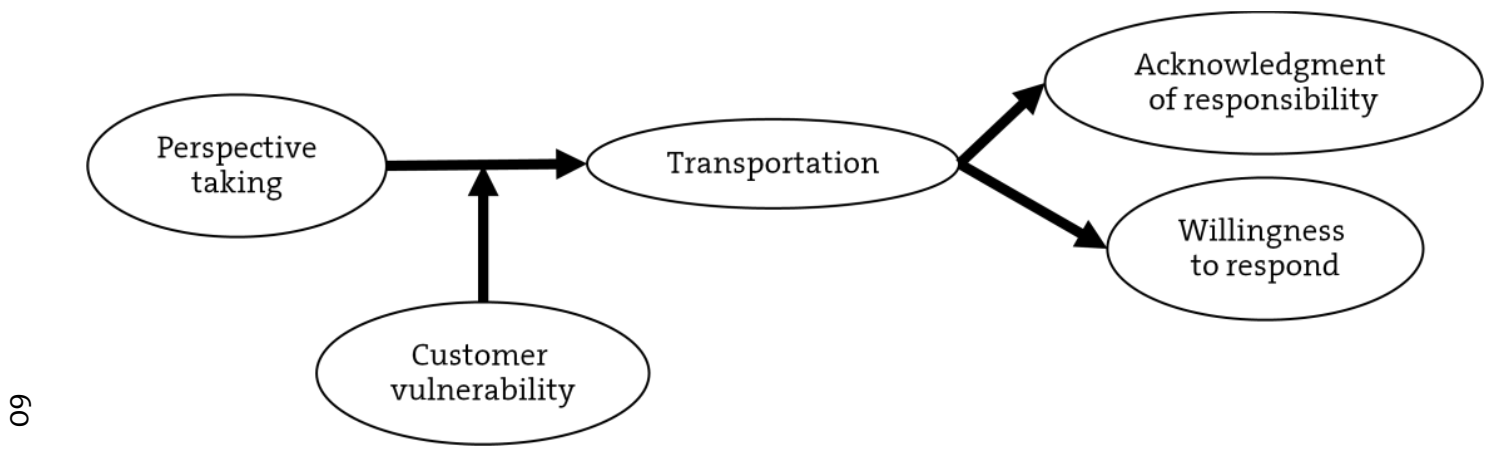

Figure 3-1

Conceptual model. 
First, recognizing the destructive effects of events described in a blog post follows directly from Lewicki and Bunker (1996); it seems relatively selfevident. Members of online communities subject to integrity violations sense a need for full disclosure about the events, as well as their consequences (Mudambi \& Schuff, 2010; Wiertz \& de Ruyter, 2007). If the events had not been destructive of integrity or the relationship, it seems difficult even to account for the posts.

Second, the cause of the violation must be identified. Previous research on integrity violations often manipulates the level of ownership by varying this dimension (Folkes \& Kotsos, 1986; Kim, Dirks, Cooper, \& Ferrin, 2006; Oliver \& DeSarbo, 1988). For example, Oliver and DeSarbo (1988) vary whether an employee did or did not pick a failed stock. True causality may be debatable though (e.g., a behaviour relates only remotely to events, the events were accidents or unintended). Even if customers feel that employee behaviour has caused the violation of their integrity, employees still could view their behaviour as relatively trivial.

Third, acknowledging responsibility for consequences is a key element of ownership. Lewicki and Bunker (1996, p. 132) argue that "taking responsibility is a key step in trust repair." It also contributes to any apologies (Bottom, Gibson, Daniels, \& Murnighan, 2002; Lewicki \& Bunker, 1996; Ohbuchi, Kameda, \& Agarie, 1989). Even if employees believe that they did not cause the events, they can influence customer relationships by acknowledging responsibility for their consequences. For example, one Best Buy customer service representative responded to a disappointed customer blogging about a broken iPhone by arranging for a replacement iPhone, even though 
the customer's insurance plan did not cover this replacement specifically (Bernoff \& Schadler, 2010). In contrast, denying all responsibility implies that the employee considers the events unimportant or insignificant for integrity perceptions or believes there were no consequences (Kim, et al., 2004). Yet in an online context, if integrity has been violated in the eye of the beholder, it has been violated (Brown \& Morgan, 2006). Disagreement only intensifies customers' negative opinions (Das \& Chen, 2007), and denial contributes to further integrity deterioration rather than repair (see Chapter 4).

Fourth, ownership studies stress the importance of willingness to respond (Harter, Schmidt, \& Hayes, 2002; Maslyn \& Uhl-Bien, 2001; Payne \& Webber, 2006; Vandenberghe, et al., 2007). Employees must be willing to invest time and energy in the relationship (Maslyn \& Uhl-Bien, 2001) and perceive that its long-term benefits are "worth" their engagement, such that their involvement and enthusiasm can lead to more customer satisfaction (Harter, et al., 2002). If employees recognize the fundamental and critical importance of strong customer relationships, they should be willing to work hard to rebuild a sense of integrity that has been violated (Payne \& Webber, 2006). Even in employee-customer encounters in which employees do not expect the relationship to continue, responsive employees should be motivated to repair an integrity violation (Vandenberghe, et al., 2007). In addition, firms generally cannot opt out of relationships online; if their relationships with bloggers sour, companies must endure the high costs of customer exits (see Chapter 4). 
In summary, the acknowledgment of responsibility and willingness to respond are both important for social media settings, because customer comments and corporate responses are subject to great visibility. In the particular case of blog posts, these efforts by employees may be visible to a very large audience (Brealy, 2010). Instilling a sense of responsibility among employees and enhancing their willingness to respond is thus clearly relevant.

\section{Transportation}

Blog posts allow employees to become closely engaged in the customer's story, so we pursue an indepth understanding of transportation, the function that underlies this effect. Slater (2002a, p. 171) notes that readers of a narrative "typically appear to be far more engrossed in the message than are readers or viewers of news stories, speeches, ads, or social science book chapters." When a reader becomes engrossed in the narrative world, he or she leaves the immediate surroundings momentarily behind. This conceptualization distinguishes transportation from similar phenomena that involve feelings of presence in mediated environments, unrelated to narratives (e.g., telepresence, Steuer, 1992), or general tendencies to be immersed into life experiences without persuasive effects (e.g., optimal experience or "flow", Csikszentmihalyi, 1992; absorption, Tellegen \& Atkinson, 1974). Being transported influences the processing of various narratives, ranging from branded advertisements (Escalas, 2004b; Wang \& Calder, 2009) to social media messages (see this dissertation), with important consequences for beliefs and intentions. It prevents readers from generating counterarguments (Slater \& Rouner, 2002). 


\section{Perspective taking}

While reading customer blogs, people might adopt three differential perspectives. Selfsufficiency suggests a perspective based on individualism and self-reliance, which generally leads to a lack of responsibility and fear of commitment (Frank, Gilovich, \& Regan, 1993; Small, Loewenstein, \& Slovic, 2007; Tang, et al., 2008; Zhou, Vohs, \& Baumeister, 2009). People with a selfsufficient perspective prefer that others do not depend on them and behave accordingly. When primed with self-sufficiency, people also are less communally motivated (Tang, et al., 2008), stay separate from others (Zhou, et al., 2009), perform socially insensitive acts (Small, et al., 2007), and make self-interested moves when faced with social dilemmas (Frank, et al., 1993).

In contrast, taking the perspective of another, such as a customer, drives other-regarding beliefs and intentions. In this mindset, people use communal norms and view the world through the cognitive lens of others (Aggarwal, 2004; Aggarwal \& Law, 2005; Aggarwal \& Zhang, 2006). Smeesters, Wheeler, and Kay (2009) show that such a perspective can instigate behaviour that supports a significant relationship. Cognitively shifting employee perspectives to the customer's view may help them acknowledge responsibility for the experiences that customers share and respond effectively to the integrity violation.

Yet blog posters also tend to be amateur authors who are personally involved (Kozinets, et al., 2010). In this case, a purely cognitive perspective is unlikely to capture the affective nature of a blogging customer's experience. Zillmann and Bryant (1994) identify empathy as a key positive affective disposition, and recent research (e.g., Nabi 
\& Krcmar, 2004; Raney, 2004; Zillmann \& Bryant, 1994) suggests that people who read personal narratives and use empathy as a significant cue display altruistic, unselfish behaviours. Furthermore, Mikulincer and colleagues (2001) demonstrate that with their empathic reaction to others, people take sides emotionally. Because empathy promotes a cognitive sense of common fate with the other party and affective togetherness, it likely enhances other-regarding perspectives in response to personal narratives. The potential impact of empathy thus demands an extension of transportation theory. An empathic perspective regards the customer as a social being and implies greater concern about the customer's thoughts and emotions. Empathic employees thus should be socially sensitive and want to work to restore the integrity of the customer.

In brief, we posit that an empathic perspective encourages transportation and its outcomes more than a self-sufficient perspective does. To determine the nature of this distinction, we undertake a conceptual investigation into empathy's robustness. That is, empathy predominantly emerges when people recognize or perceive a particular relationship between the narrator and the narrative events (Dunlop, et al., 2008a). In the case of integrity violations, empathy gets triggered by the recognition of the customer's vulnerability to the violation. Accordingly, we predict that empathy is triggered most often when the employee recognizes that the customer is vulnerable to financial loss due to the integrity violation. In this context, vulnerability refers to the employee's belief that the customer is suffering from the integrity violation and that the financial consequences are severe (R. C. Mayer \& Davis, 1999). Information about high vulnerability should evoke stronger empathic 
reactions. As De Wit, Das, and Vet (2008) propose, the presence of such information during the experience of affect also can influence transportation, independent of cognitive appraisals. In contrast, low vulnerability exhibits an inverse relationship with empathy (Greenwood, 2007). We therefore concentrate on the transient recognition of customer vulnerability and the way it moderates the effect of an empathic perspective.

\section{Hypotheses development}

Before the rise of social media, toll-free numbers and call centre agents were the primary responders to customer complaints about integrity violations. Expressions of dissatisfaction through such media involve dyadic interactions, whereas blog posts usually are vented widely and publicly online. Because blog posts also are narratives, in which characters experience causally connected events, customers are the protagonists of their own stories (Adaval \& Wyer Jr., 1998), and empathy for these characters may drive transportation (Green \& Brock, 2000, 2002). Simultaneously, readers likely experience inhibition of negative cognitive responses, such that they are not inclined to argue against narrative-based beliefs and intentions. After they have been transported, people generally exhibit positive responses (Polichak \& Gerrig, 2002) and often change their view to support the protagonist (Slater, 2002b). We extend these ideas to determine if transportation also underlies a sense of responsibility and an intention to respond to blog complaints. People who read a blog post and adopt the perspective of the thoughts and emotions of the story characters should be more transported into the consumption experience than people who are only cognitively affected. The greater transportation 
then strengthens willingness to take responsibility and respond.

We also posit that an empathic perspective drives these outcomes, through transportation. Empathy here is not a stable trait or service outcome but rather a mental driver or transitory mindset. Using mental triggers that momentarily improve a person's perspective should influence psychological ownership as well. It may be possible to influence employees' acknowledgment of responsibility just by varying their perspective (constant blog posts, ceteris paribus). Because descriptions of integrity violations in blogs usually are personal, an activated empathic perspective may make employees feel relatively more responsible for the events, compared with their response to a purely cognitive perspective. An empathic perspective also should imply that employees temporarily share the thoughts and emotions of customers and get transported into the narrative, which could have desirable impacts on their willingness to respond. An activated perspective of the self instead should mean that self-sufficient employees are less transported into the blog post, compared with employees with empathic or cognitive perspectives; they also should be less likely to feel responsible or respond to the integrity violation. We hypothesize:

$\mathbf{H}_{1}$ : An empathic perspective leads to greater (a) acknowledgment of responsibility and (b) willingness to respond than a cognitive or self-sufficient perspective.

$\mathbf{H}_{2}$ : Transportation mediates the effect of an empathic perspective on

(a) acknowledgment of responsibility and

(b) willingness to respond. 
Knowing the customer's financial vulnerability also might differentially influence the likelihood of employee transportation. Following Dunlop, Wakefield, and Kashima (2008a), we predict that empathy is mainly triggered by employees' recognition of the customer's financial vulnerability to the integrity violation, perhaps based on the customer risk profile. Arguably, higher customer vulnerability should lead to more employee transportation, because employees should understand the violation accusations in the blog posts. These employees may be able to draw on this recognition of vulnerability to empathize more with the characters (i.e., blogging customers) and be transported more deeply into the blog post. In contrast, employees who encounter a customer who is relatively less financially vulnerable may sense greater distance from the customer's violation accusation, because of the greater effort they must exert to create an empathic perspective. Prior knowledge about customer financial vulnerability thus should interact with an empathic perspective to influence transportation, and we hypothesize
$\mathrm{H}_{3}$ :
As a customer's financial vulnerability increases, employee responsiveness to empathy increases, thus increasing the effect of an empathic perspective on transportation.

We test these three hypotheses with an extensive pre-test and field study. 


\section{Empirical study}

\section{Pre-test}

To test our hypotheses, we constructed priming techniques for empathy and self-sufficiency. In a pre-test, we examined whether they appropriately primed these mental models. To rule out the possibility that either technique failed to prime the desired concept, we also included a neutral priming condition. The focal blog post contained a customer accusation of an integrity violation, and the pre-test also determined whether the blog post met four necessary criteria to produce transportation and ownership effects. First, following Green and Brock (2002), we checked whether participants perceived the blog post as a narrative. Second, for them to assess ownership, participants should be able to recognize the nature of the violation. Third, the blog post revealed the cause for the integrity violation, such that it could be attributed to both customer and employee. Fourth, the blog post needed to feature unfavourable consequences for the customer, for which an employee plausibly could acknowledge some responsibility.

In the priming manipulation, participants solved a puzzle in which they had to find words that activated empathy, self-sufficiency, or neither. Therefore, the pre-test used an empathy versus selfsufficiency versus neutral priming betweensubjects design. The participants were randomly assigned to the priming conditions and then asked to read an online blog post in which a customer described an integrity failure during a financial service encounter. Finally, we assessed whether the priming techniques were effective and the blog post met the criteria. 
Participants

From a medium-sized Dutch university, a sample of 105 business graduate students participated; they should have a reasonable understanding of a financial services context. The participants were 23 years of age on average (18-29 years), and $55.2 \%$ were women.

\section{Priming and material}

To prime the participants, we used three computer-generated word search puzzles that each participant completed at the beginning of the experimental session. Solving a puzzle offers a valid technique for priming participants to think deeply about a particular concept-ranging from achievement to affiliation (Bargh, 1997; Bargh \& Barndollar, 1996; Bargh, Gollwitzer, Lee-Chai, Barndollar, \& Trötschel, 2001)—without direct instructions. The puzzles were 15 letters long and 15 letters wide, and words could be spelled horizontally, vertically, or diagonally. Below each puzzle, we provided a list of 12 words embedded in the matrix, and in all cases, the same set of 6 neutral words appeared in this list (i.e., book, bottom, building, green, jump, and metal). 
In the empathy priming condition, the other 6 words were those used by Mikulincer and colleagues (2001)—compassion, moved, softhearted, sympathy, tender, and warm (Figure 3-2)to connote empathy for others.

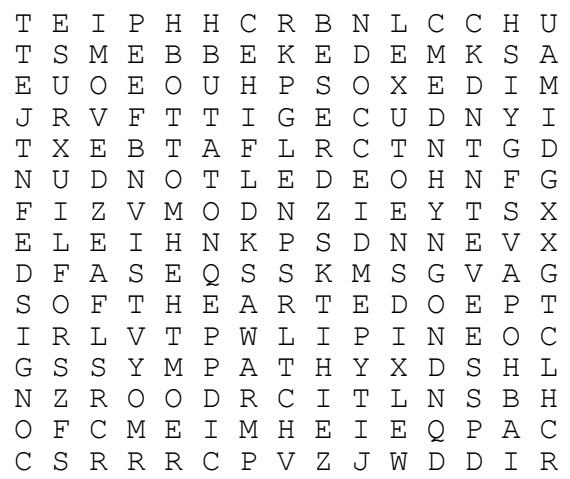

book

bottom

building

compassion

green

jump

metal

moved

soft-hearted

sympathy

tender

warm

Figure 3-2

Empathy word search puzzle. 
The self-sufficiency words came from Vohs, Mead, and Goode (2006): capital, check, profits, raise, revenues, and wealthy (Figure 3-3). They should activate self-sufficiency.

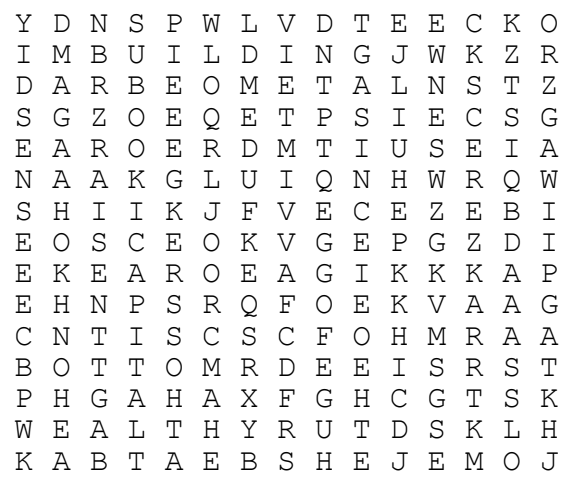

book

bottom

building

capital

check

green

jump

metal

profits

raise

revenues

wealthy

\section{Figure 3-3}

Self-sufficiency word search puzzle. 
In the neutral condition, the 6 remaining words had no specific connotation (i.e., going, is, printer, purple, top, and win; see Figure 3-4). The neutral words also came from Vohs, Mead, and Goode (2006).

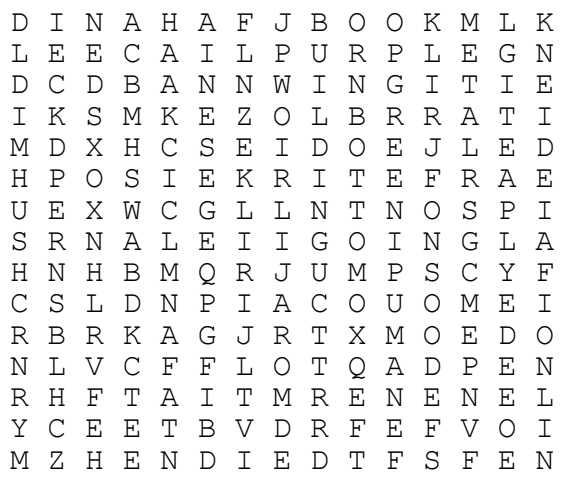

book

bottom

building

going

green

is

jump

metal

printer

purple

top

win

Figure 3-4

Neutral word search puzzle. 
In the selected blog post, a customer "Paul" told the story of losing his retirement savings by being sold an investment fund that evaporated (see Appendices, pp. 209-210). We set the scene in a financial service context for three reasons. First, it is hard for customers to judge the quality of financial products, which are complex and difficult to understand, so they often rely on trusted financial advisers. Second, both the company and a wider community realistically might be alerted simultaneously through blogs after an integrity violation. Third, Green, Brock, and Kaufman (2004) claim that a topic's popularity may signal its capacity to transport readers, and financial services involve four of the nine absolute interests: chaos, destruction, money, and power (for the complete list, see Schank, 1979).

\section{Measures}

To assess the degree of empathy that the word search puzzles primed, we adapted Davis's (1983) empathic ability items to make them appropriate to our blog post context (see Appendices, p. 223).

To check the narrative format, we asked participants to respond to an instrument about the blog post's storytelling features, using seven semantic scales (see Appendices, p. 223).

Regarding whether the participants identified the nature of the violation, we asked them what the accusation brought into question: "primarily the financial adviser's competence (e.g., knowledge of finance)," "primarily the financial adviser's integrity (e.g., willingness to bend the rules)," or "neither of the above."

We relied on standard measures of causal attribution of blame and acknowledgment of 
responsibility (Fenigstein \& Levine, 1984; Folkes \& Kotsos, 1986; Pham, Goukens, Lehmann, \& Stuart, 2010). That is, we assessed causal attribution of blame by asking participants to divide 100 points across the customer and the financial adviser. They completed a similar task for acknowledgment of responsibility, in which they assessed the extent to which they thought the financial adviser should take responsibility for the consequences of selling the investment fund to the customer (up to 100\%).

We also tested whether participants were equally susceptible to empathy and self-sufficiency. The different participant groups would need to be homogeneous in this respect for us to compare priming technique effects. Therefore, we used Tang and colleagues' (2008) love of money scale to measure individual aspirations for and attitudes toward money and Reynolds's (2008) moral attentiveness scale to measure their tendency to over-represent or exaggerate moral experiences (see Appendices, p. 225).

\section{Pre-test results}

\section{Material checks}

Responses to the material checks revealed that the blog post met all four criteria. To check the narrative format criterion, we examined whether the participants correctly identified the narrative blog post format. They rated it significantly above the neutral midpoint of the aggregated scale $\left(t_{(104)}=16.94, p<.001\right)$, which indicated that they perceived the format as significantly narrative $(M=5.48, \mathrm{SD}=.90)$, across all priming conditions $\left(F_{(2,102)}=1.91, p=.153\right)$. For the identification of the violation, almost all participants (102 of 105) 
reported that they thought that the financial adviser's integrity came into question. The remaining responses did not show a correlational pattern across priming conditions $\chi_{(4)}^{2}=3.68$, $p=.452){ }^{3}$ We also assessed whether the participants could attribute blame to the financial adviser or the customer; blame was convincingly attributed to neither ( $M=51.43, \mathrm{SD}=35.58$, ranging from 0 to 100$)$. Thus, participants across all priming conditions were equally ambiguous in attributing blame $\left(F_{(2,102)}=2.06, p=.133\right)$. Finally, we concluded that the acknowledgment of responsibility criterion was met. The priming should have influenced this perception, so we performed a paired-samples $t$-test to compare attributions of blame against the acknowledgment of responsibility in the neutral priming condition. The test revealed that participants' acknowledgment of responsibility was significantly greater than their attribution of blame (mean difference $=19.41, \mathrm{SE}=4.53 ; t_{(33)}=4.29, p<.001$ ).

\section{Priming checks}

Table 3-1 contains the dependent and control variables' average scores, standard deviations, reliabilities, and intercorrelations.

\footnotetext{
${ }^{3}$ We conducted separate analyses for the 102 participants. The results showed a pattern similar to that for the 105 participants reported here.
} 


\section{Table 3-1}

Pre-test: Descriptive statistics.

\begin{tabular}{lllll}
\hline & & $M(\mathrm{SD})$ & $\alpha$ & 1 \\
\hline 1 & Empathy & $4.01(.82)$ & .73 & \\
& Love of money & $5.99(.66)$ & .75 & $.25^{* *}$ \\
& Moral attentiveness & $3.73(1.01)$ & .90 & \\
\hline${ }^{* *} p<.01$. & & &
\end{tabular}

We first verified if participants across all conditions were equally susceptible to empathy and selfsufficiency. We conducted regression analyses with age, love of money, and moral attentiveness as predictors and performed a $t$-test of gender on perceived empathy. The relationships were not significant $(t<2.00, p>.05)$, so heterogeneity was not an issue.

Our successful priming also was reflected in the effect on measured empathy $\left(F_{(2,102)}=17.70, p<.001\right.$; $\left.\eta^{2}=.258\right)$. An analysis of variance revealed that the empathy word search puzzle primed empathy to a greater degree than the neutral (mean difference=.84, SE=.22, $p<.001$ ) or self-sufficiency (mean difference=1.30, SE=.22, $p<.001$ ) puzzles. Neutral priming also resulted in higher empathy levels than did self-sufficiency priming (mean difference $=.46, \mathrm{SE}=.23, p<.05)$.

To provide a preliminary test of $\mathrm{H}_{1 \mathrm{a}}$, we conducted another analysis of variance $\left(F_{(2,} \quad{ }_{102)}=15.49, \quad p<.001 ; \quad \eta^{2}=.233\right)$. Comparing participants primed with empathy against those in the other priming conditions, we found that the empathy-primed participants ascribed more responsibility to the financial adviser than did participants in the neutral (mean difference $=24.23$, $\mathrm{SE}=7.53, \quad p<.01$ ) or self-sufficiency (mean 
difference=41.34, SE=7.47, $p<.001$ ) conditions. The difference between the neutral and self-sufficiency conditions also was positively significant (mean difference=17.11, SE=7.58, $p<.05$ ). However, considering the sample and lack of a cognitive group, the pre-test did not provide an adequate test of $\mathrm{H}_{1 \mathrm{a}}$.

\section{Study method}

We designed the main study to examine the effect of an empathic versus a cognitive or selfsufficient employee perspective across differing levels of the customer's financial vulnerability. The experiment was conducted in an employee training context, such that we introduced it as an exercise for assessing customer vulnerability. Before reading a customer profile, participants received a word search puzzle, reportedly to help them relax while the training session was being prepared. Unbeknownst to participants, we primed their empathy or self-sufficiency with these puzzles, similar to the procedure in the pre-test. To confirm the priming had a sufficient impact on participants' perspectives, we retained a cognitive perspective group that did not complete a prime. The participants then read a customer risk profile, which indicated prior knowledge about that customer's financial vulnerability, whether high, moderate, or low. Next, participants chose an investment fund to offer to the customer but then discovered the customer had gotten into severe financial trouble after accepting the offer, as recounted in a blog post supposedly written by this customer. All participants were instructed to respond to the blog post according to standard procedures for customer feedback. This instruction stimulated participants to take the customer's perspective. After reading the 
blog post, participants completed a questionnaire. Thus, our study used a 3 (perspective: empathic, cognitive, or self-sufficient) $\times 3$ (customer vulnerability: high, moderate, or low) factorial design, with participants randomly assigned to the different groups. After all participants finished the study, we informed them of the goal of the experiment and answered any questions.

\section{Participants}

Branch managers from a global Fortune 100 banking group $(n=350)$ participated in this study; they represent an initial level of contact who have the authority to repair customer trust (according to a 2009 internal document from the banking group). These professional experts averaged 44 years of age (from 25-60 years), had 20 years of business experience, and were primarily of Belgian nationality (98.3\%). ${ }^{4}$ A small minority (1.2\%) had received no diploma, $25.2 \%$ earned a high school diploma, $47.4 \%$ received a college degree, and $26.2 \%$ graduated from university. The sample consisted mostly of men $(75.9 \%)$, reflective of the $70 \%$ male workforce. The sample represented 246 bank branches.

\section{Priming, material, and manipulation}

As we noted previously, we used the empathy and self-sufficiency priming from the pre-test (see Figures 3-2 and 3-3). The cognitive group did not get a puzzle. After the experiment, we asked

\footnotetext{
${ }^{4}$ Because the banking group's working languages included both Dutch and French, we carried out International Test Commission translation and back-translation procedures on all materials and instruments (Hambleton, 2001; Hambleton, Yu, \& Slater, 1999; Mullen, 1995).
} 
participants to respond to a question about the purpose of the word search puzzle. Their responses revealed that the intent was successfully hidden; none of the participants correctly guessed the puzzle's purpose. Following the priming, the participants read the following excerpt:

Paul has accepted your offer. Please read the following blog post by Paul. While reading this blog post, apply standard procedures for dealing with customer feedback. Think about what happened. Place yourself in Paul's shoes.

This instruction thus stimulated cognitive perspective taking, in line with Green and Brock (2000). The pretested blog post brought the employee's integrity into question (see Appendices, pp. 209-210).

To manipulate customer financial vulnerability, we applied the MiFID ("Markets in Financial Instruments Directive," 2009); this European Commission directive prescribes, among other things, that when an employee of a financial institution gives advice, he or she must consider the customer's profile, including financial situation, investment aims, and investment knowledge and experience. The MiFID also obliges financial institutions to classify all customers according to their financial vulnerability. ${ }^{5}$ Therefore, we used three customer profiles to describe them, as follows:

5 High, moderate, and low vulnerability are feigned designations. The names presented represented stock marketsensitive information. 
"High"

Paul has some money in a savings account, and he buys mainly savings certificates. Paul only wants investments that yield a guaranteed or predictable return. He thinks investing in stocks and shares is in fact "gambling." Thus, things like shares are lost on him.

\section{"Moderate"}

One quarter of Paul's professional income goes into paying off his house and his car. Furthermore, he puts money in the bank, and he contributes to a pension fund. Safe, fixed-interest savings certificates and funds make up the principal part of his investment portfolio. On occasion, he dares to buy an equity fund with money he does not need right away.

"Low"

Paul devotes his investment strategy completely to "opportunities." Shares make up three-quarters of his investment portfolio. Paul knows that shares can turn out badly sometimes, but that does not put him off. On the contrary, he interprets a drop in prices as an opportunity to buy more. In his bond portfolio, he wants to have it all.

\section{Measures}

Except for a slight adaptation to the particular context, the acknowledgment of responsibility measure was identical to that used in the pre-test. The willingness to respond scale consisted of three Likert-type statements that measured participants' desire to continue a relationship with a customer, as well as their willingness to sustain the relationship over time. The scale came from De Wulf, OdekerkenSchröder, and Iacobucci (2001). Because the composite reliability was inadequate for the complete scale $(\alpha=.66)$, we dropped the item "Even if Paul would be more difficult to retain, I would still keep trying." The reliability for the remaining two 
items was acceptable $(\alpha=.76, \rho=.61, p<.001$; see Appendices, p. 228).

Because one of our primary aims was to test whether transportation could predict narrativebased responsibility acknowledgment $\left(\mathrm{H}_{2 \mathrm{a}}\right)$ and willingness to respond $\left(\mathrm{H}_{2 \mathrm{~b}}\right)$, participants also completed the transportation questionnaire developed by Green and Brock (2000, see Appendices, p. 228). They responded to the items on Likert-type scales ranging from "strongly disagree" to "strongly agree."

As in the pre-test, we assessed participants' homogeneity. However, the priming words could be interacting with branch managers' customeroriented abilities, so the homogeneity measures we used in the pre-test were insufficient for our purposes here. In addition to the love of money (Tang, et al., 2008) and moral attentiveness (Reynolds, 2008) measures, we therefore compared participants according to the different conditions in Saxe and Weitz's (1982) selling orientationcustomer orientation scale (as shortened by Periatt, LeMay, \& Chakrabarty, 2004), which provides a measure of the general tendency to meet customer needs (see Appendices, pp. 222 and 227).

\section{Study results}

Manipulation checks

Participants answered two manipulation check questions to confirm the customer vulnerability manipulation (high, moderate, or low). The first question pertained to the estimated risk associated with the investment fund they might offer. After reading a customer profile, participants chose one of their banking group's investment funds and 
indicated the risk they believed it represented, according to the official European Union (EU) risk classification, which ranged from 0 (no risk) to 5 (high risk). The second question checked whether the participants recognized the customer profile: "You have just read Paul's customer profile. What level of financial vulnerability does Paul have?" The possible answers were high, moderate, or low.

Responses to the two checks revealed that the manipulation was successful. Participants' risk estimations differed significantly among the high $(M=4.04, \mathrm{SD}=1.22)$, moderate $(M=2.60, \mathrm{SD}=1.60)$, and low $\left(M=1.53, \mathrm{SD}=1.27, F_{(2,347)}=104.61, p<.001, \eta^{2}=.366\right.$; simple contrasts: $\mathrm{SE}=.17, \quad p<.001)$ customer vulnerability conditions. Of 350 participants, 343 answered the customer profile question correctly $\left(\chi_{(6)}^{2}=966.03, \quad p<.001, \quad \varphi=1.63\right)$. We excluded 7 participants who wrongly answered the customer profile question, because they likely read the profile partially or carelessly.

Another question, adapted from Green and Brock (2000), determined participants' task understanding while they read the blog post. They answered two Likert-type items, anchored by "strongly disagree" to "strongly agree," regarding whether they "become Paul" and "think about what happened" ( $\rho=.79, \quad p<.001)$. The average was significantly above the neutral midpoint of the aggregated scale $\left(t_{(349)}=210.73, p<.001\right)$ and did not differ across priming conditions $\left(F_{(2,}, 347\right)=1.05$, $p=.351)$. That is, all participants tried to take Paul's perspective on the narrative events $(M=3.98$, $\mathrm{SD}=.18$ ), and the task was well understood.

Table 3-2 contains the average scores, reliabilities, and intercorrelations of the dependent and control measures. 


\section{Table 3-2}

Study: Descriptive statistics.

\begin{tabular}{llllllll}
\hline & & $M(\mathrm{SD})$ & $\alpha$ & 1 & 2 & 3 & 4 \\
\hline 1 & Acknowledgment of responsibility & $42.86(25.17)$ & & & & & \\
2 & Willingness to respond & $3.91(.91)$ & .77 & & & & \\
3 & Transportation & $3.22(1.29)$ & .76 & $.30^{* *}$ & $.16^{* *}$ & & \\
& Love of money & $3.91(1.08)$ & .82 & & & & $.26^{* *}$ \\
& Moral attentiveness & $3.63(1.09)$ & .89 & $.12^{*}$ & $.21^{* *}$ & $-.14^{* *}$ & \\
& Customer orientation & $6.30(.67)$ & .87 & & & & $-.29^{* *}$ \\
4 & Selling orientation & $2.16(1.00)$ & .73 & $-.15^{* *}$ & & & \\
\hline
\end{tabular}

${ }^{*} p<.05 ;{ }^{* *} p<.01$. 
We also verified whether the collected demographics and homogeneity measures influenced our dependent measures. In three multiple regression analyses, we took age, business experience, bank branch, love of money, moral attentiveness, customer orientation, and selling orientation into consideration as potential predictor variables for acknowledgment of responsibility, willingness to respond, and transportation. The models were significant for acknowledgment of responsibility $\left(F_{(7,335)}=2.86, \quad p<.01, R^{2}=.056\right)$ and transportation $\left(F_{(7,335)}=3.82, p<.01, R^{2}=.074\right)$. Age had a positive effect on acknowledgment of responsibility $\left(\beta=.24, t_{(1)}=2.12, \quad p<.05\right)$, whereas selling orientation had a negative effect $\left(\beta=-.13, t_{(1)}=-2.22, p<.05\right)$. Furthermore, age $(\beta=.23$, $\left.t_{(1)}=2.06, \quad p<.05\right)$ and moral attentiveness $(\beta=.18$, $\left.t_{(1)}=3.43, \quad p<.01\right)$ both had positive effects on transportation. Analyses of variance comparing nationalities, working languages, levels of education, and genders with regard to their effects on acknowledgment of responsibility, willingness to respond, and transportation revealed that gender significantly influenced acknowledgment of responsibility $\left(F_{(1,331)}=4.25, p<.05, \eta^{2}=.013\right)$, in that women $\quad(M=48.07, \quad \mathrm{SD}=20.51) \quad$ acknowledged significantly more responsibility than men $(M=42.22, \mathrm{SD}=26.11)$. The remaining relationships were not significant (regression analyses: $t<1.90$, $p>.06$; analyses of variance: $F<1.80, p>.10)$. Age, selling orientation, moral attentiveness, and gender therefore served as covariates in our subsequent analyses. 


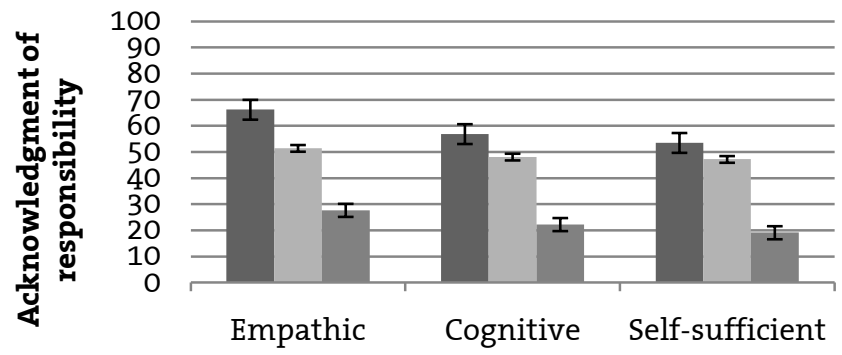

- High vulnerability $\quad$ Moderate vulnerability

- Low vulnerability

\section{Figure 3-5}

Study: Acknowledgment of responsibility for different perspective and customer vulnerability combinations.

The error bars show the standard error.

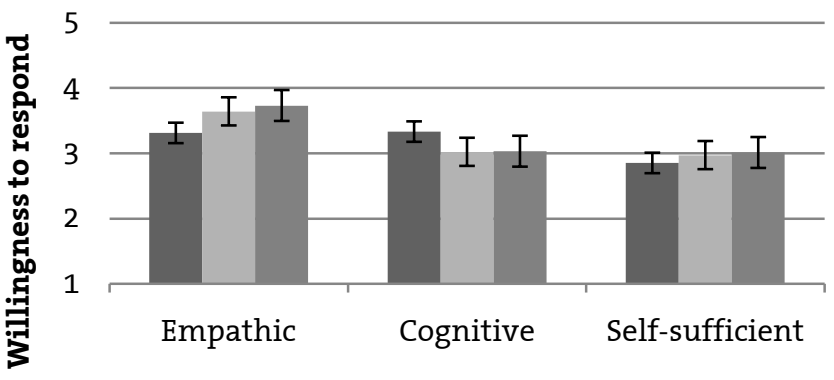

- High vulnerability $\quad$ Moderate vulnerability

- Low vulnerability

\section{Figure 3-6}

Study: Willingness to respond for different perspective and customer vulnerability combinations.

The error bars show the standard error. 
Hypotheses tests

Figures 3-5 and 3-6 shows the average scores and standard errors for acknowledgment of responsibility and willingness to respond across empathic, cognitive, and self-sufficient perspectives, as well as customer vulnerability conditions.

To test $\mathrm{H}_{1 \mathrm{a}}$, we conducted an analysis of covariance to examine responsibility acknowledgment across the perspective conditions. The analysis indicated a significant perspective effect $\left(F_{(2,330)}=5.88, p<.05, \eta^{2}=.034\right)$. According to simple contrast tests, acknowledgment of responsibility was higher with an empathic than with a cognitive (mean difference $=6.03, \mathrm{SE}=2.57$, $p<.05$ ) or self-sufficient (mean difference $=8.50$, $\mathrm{SE}=2.54, p<.01)$ perspective. There was no significant difference between cognitive and self-sufficient perspectives (mean difference $=2.47, \quad \mathrm{SE}=2.52$, $p=.327)$.

We also hypothesized that willingness to respond would differ across these perspectives $\left(\mathrm{H}_{1 b}\right)$. The relevant analysis of covariance revealed a significant difference among perspectives $\left(F_{(2,330)}=5.88, p<.01, \eta^{2}=.034\right)$. Willingness to respond was higher for empathic than for cognitive (mean difference $=.44, \mathrm{SE}=.17, \quad p<.05)$ and self-sufficient (mean difference $=.62, \mathrm{SE}=.17, p<.001$ ) perspectives. Willingness to respond was also significantly different between the cognitive and self-sufficient perspectives (mean difference $=.18, \mathrm{SE}=.17, p=.280$ ). In summary, the results support $\mathrm{H}_{1 \mathrm{~b}}$.

Transportation was the primary dependent variable for the tests of $\mathrm{H}_{2}$ and $\mathrm{H}_{3}$. A $3 \times 3$ analysis of covariance showed significant differences among perspectives $\left(F_{(2,330)}=15.72, p<.001, \eta^{2}=.087\right)$ and customer vulnerability conditions $\left(F_{(2,330)}=12.22\right.$, $p<.001, \eta^{2}=.069$ ). According to the simple contrasts, 
participants primed to empathize with the customer experienced more transportation than those who took a cognitive (mean difference=.29, $\mathrm{SE}=.11, \quad p<.05)$ or self-sufficient (mean difference $=.62, \quad \mathrm{SE}=.11, \quad p<.001) \quad$ perspective. Participants with a cognitive perspective also were more transported than those with a self-sufficient perspective (mean difference=.33, SE=.11, $p<.01$ ). To test whether transportation explains the effect of empathic perspective on acknowledgment of responsibility and willingness to respond, we conducted mediation analyses (as derived from MacKinnon, Lockwood, Hoffman, West, \& Sheets, 2002; Zhao, Lynch Jr., \& Chen, 2010). The Sobel tests support $\mathrm{H}_{2 \mathrm{a}}(Z=4.19, p<.001)$ and $\mathrm{H}_{2 \mathrm{~b}}(Z=2.74, p<.01)$.

Our successful priming and manipulation also were reflected in the interaction effect on transportation $\left(F_{(4,330)}=3.10, p<.05, \eta^{2}=.036\right)$, as we detail in Table 3-3. Simple contrast tests revealed that high customer vulnerability led to more transportation than moderate or low vulnerability with an empathic perspective compared with a selfsufficient perspective, in support of $\mathrm{H}_{3}$. However, customer vulnerability levels did not result in different transportation when participants took a cognitive perspective (see Figure 3-7). 


\section{Table 3-3}

Study: Simple contrasts for perspective and customer vulnerability combinations on transportation.

\begin{tabular}{|c|c|c|c|c|c|c|c|c|c|c|}
\hline & \multirow{2}{*}{ Perspective } & \multirow{2}{*}{ Customer vulnerability } & \multicolumn{8}{|c|}{ Mean difference (SE) } \\
\hline & & & 1 & 2 & 3 & 4 & 5 & 6 & 7 & 8 \\
\hline 1 & Empathic & High & & & & & & & & \\
\hline 2 & & Moderate & $.47(.17)^{* *}$ & & & & & & & \\
\hline 3 & & Low & $1.02(.21)^{* * *}$ & $.55(.20)^{* *}$ & & & & & & \\
\hline 4 & Cognitive & High & $.78(.19)^{* * *}$ & $.31(.19)$ & $.24(.22)$ & & & & & \\
\hline 5 & & Moderate & $.73(.19)^{* * *}$ & $.27(.19)$ & $.28(.22)$ & $.05(.21)$ & & & & \\
\hline 6 & & Low & $.83(.16)^{* * *}$ & $.36(.16)^{*}$ & $.19(.20)$ & $.05(.18)$ & $.09(.18)$ & & & \\
\hline 7 & Self-sufficient & High & $.82(.18)^{* * *}$ & $.35(.17)^{*}$ & $.20(.21)$ & $.04(.20)$ & $.08(.20)$ & $.01(.17)$ & & \\
\hline \multirow[t]{2}{*}{8} & & Moderate & $1.19(.18)^{* * *}$ & $.72(.18)^{* * *}$ & $.17(.22)$ & $.41(.20)^{*}$ & $.46(.20)^{*}$ & $.36(.17)^{*}$ & $.38(.19)$ & \\
\hline & & Low & $1.47(.17)^{* * *}$ & $1.00(.17)^{* * *}$ & $.46(.21)^{*}$ & $\begin{array}{l}.69 \\
(.19)^{* * *}\end{array}$ & $\begin{array}{l}.74 \\
(.19)^{* * *}\end{array}$ & $\begin{array}{l}.64 \\
(.16)^{* * *}\end{array}$ & $.66(.18)^{* *}$ & $.28(.18)$ \\
\hline
\end{tabular}

${ }^{*} p<.05 ;{ }^{* *} p<.01 ;{ }^{* * *} p<.001$ 


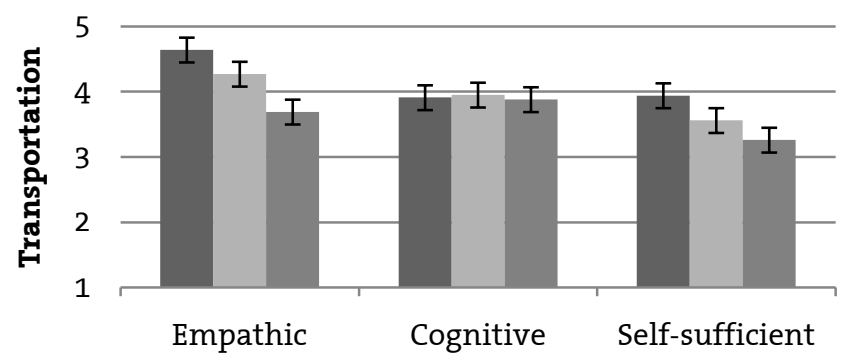

- High vulnerability $\quad$ Moderate vulnerability

- Low vulnerability

\section{Figure 3-7}

Study: Transportation for different perspective and customer vulnerability combinations.

The error bars show the standard error.

\section{Discussion}

This chapter's results contribute to existing research in two main ways. First, we reveal that different perspectives either encourage or discourage transportation. The effect of these different perspectives on responsibility acknowledgment and responding intentions thus is mediated by transportation. Relative to a selfsufficient perspective, employees with an empathic or cognitive perspective experience more transportation and feel greater moral obligation to respond to blog posts or engage in online conversations about the company. Our study also confirms that an empathic perspective is more effective than a cognitive perspective in creating the desired mindset, such that it prepares employees to attempt to restore integrity when they address the narratives. We thus add detail to Green and Brock's (2000) findings of a positive effect 
of perspective taking on transportation and expand scholarly understanding of the ways narratives affect beliefs and intentions.

Second, we examine the impact of a boundary condition related to perspective taking, namely, customers' financial vulnerability. In a transporting setting, different customer vulnerability levels significantly affect perspective priming. The knowledge that a customer is highly vulnerable to the consequences of integrity violation influences the transportation experience, such that a narrative with an example of an integrity violation becomes even more compelling if the reader adopts an empathic perspective. This finding of an association among customer vulnerability, greater empathy, and deeper transportation by employees reinforces Dunlop, Wakefield, and Kashima's (2008a) theory.

\section{Limitations}

Although by definition field research cannot provide the same extent of control as a laboratory study, our experiment was designed and conducted meticulously. The external validity appears acceptable, yet still, the research contributions must be evaluated in light of the study limitations.

First, we base our manipulation of a selfsufficient perspective on experiments by Vohs and colleagues (2006), who use monetary priming. In line with their interpretation, we find that if people believe they are more self-sufficient, they will be less engrossed in the blog post and less likely to feel they should help others. However, Aggarwal (2004) conceptualizes a similar perspective as "quid pro quo," with an exchange relationship in mind. 
Second, the participants in the pre-test should have knowledge of this context, but they may have had limited experience evaluating investment funds. This lack of experience could evoke excessive feelings of empathy for the customer and an overestimation of the financial adviser's responsibility. Therefore, we replicated the pre-test in the main study with participants who had extensive experience with investment funds.

Third, we only consider blog posts, though customers share integrity violations through other social media too (e.g., status updates on Facebook, video logs on YouTube). Green and Brock (2002) argue that when narratives trigger fewer senses, recipients must exert more imaginative effort, which results in more transportation. Reading a blog post thus might provoke greater transportation than watching a video log. Yet Polichak and Gerrig (2002) also suggest that audiovisual stories generate different participatory responses than written stories, because they trigger both sight and hearing. 
In brief, online customer narratives can have serious destructive potential, so employees must assume ownership of the issues described in these narratives. Transportation prepares employees to respond effectively to complaints of integrity violations in blog posts. Although the outcomes are conditional on customer vulnerability, employees with an empathic perspective seem to display the highest levels of transportation into the customer experience, which enhances their sense of responsibility and willingness to respond. 
Chapter 4 is based on

van Laer, T., \& de Ruyter, K. (2010). In stories we trust: How narrative apologies provide cover for competitive vulnerability after integrity-violating blog posts. International Journal of Research in Marketing, 27(2), 164-174. 


\section{4 \\ IN STORIES WE TRUST}

How narrative apologies provide cover for competitive vulnerability after integrity-violating blog posts

\section{Introduction}

Blog posts keep gaining in importance and are dramatically influencing the way consumers process and share information (Woodside, et al., 2008) and make purchase decisions (Pew Internet \& American Life Project, 2006). It is these stories that present-day consumers have come to trust. Recent and widespread online violations of integrity and consumers' voicing of discontent form a threat to consumers' confidence in companies (Ward \& Ostrom, 2006). Even unconfirmed posts can develop rapidly into stories with serious destructive potential and are a source of severe competitive vulnerability because consumers switch to competitors at virtually no cost to them (Elsner, Heil, \& Sinha, 2010). Well-known companies, including American Airlines and Kryptonite, have experienced massive exits by consumers based on posts on anonymous blogs. For such companies, a strategy of reticence, or hoping that the storm of negative word-of-mouth will just blow over, is no longer effective in the changing competitive landscape. Rather, the highly reactive blogosphere demands a quick and appropriate response in the 
blog's comment section to avoid the further erosion of consumer trust and subsequent loss of market share (Li, Bernoff, \& McHarg, 2004). Trusov, Bucklin, and Pauwels (2009) show that an effective marketing strategy online differs from traditional public relations strategies, such as issuing press releases or comments from a spokesperson. Therefore, an in-depth assessment of effective responses to integrity violations, such as those voiced in consumer blogs, is crucial from a competition perspective.

Interestingly, recent theory on social interaction in an offline setting has predicted that a response that denies a breach of integrity restores trust more effectively than one that apologizes (for a review, see Snyder \& Stukas Jr., 1999). Empirical evidence for this prediction is scant and limited to studies by one research team (Ferrin, et al., 2007; Kim, et al., 2006; Kim, et al., 2004). These studies focus almost exclusively on the content of responses (i.e., apology or denial) that are presented in factual, analytical formats. Blog posts differ in several ways: (1) the blog post format is more narrative and experiential (Delgadillo \& Escalas, 2004), (2) the sheer number of blog posts makes it hard to respond to and control their competitive impact (Gartner Research, 2007; The Economist, 2006), and (3) blog posters tend to be non-professional authors who are personally involved (Kozinets, et al., 2010). Current research on integrity restoration therefore offers little guidance regarding whether an overtly persuasive, fact-based, analytical response format is appropriate or whether the firm instead should adopt a covertly persuasive, first-person narrative style in its response.

In addressing this issue, we begin our literature review by briefly describing integrity violations and 
their impact on consumers' trusting beliefs and subsequent intentions to switch. We then sketch the processing of analytical responses that aim to restore integrity perceptions and reduce intentions to switch. Next, we turn to the processing of narratives. We make a case for the concept of "transportation" as the mechanism underlying narrative-based integrity restoration. Having laid the groundwork for our hypotheses, we report on three separate studies in which we aim to make three substantive contributions to prior research.

First, we show in Study 1 which combinations of response content and format work best under what conditions. Considering the potential competitive impact of blog posts, we also establish that integrity perceptions lead to outcomes that are relevant for competition: lower intentions to switch. In relation to integrity-violating blog posts, we posit that not only the content but also the format of a response contribute to an effective restoration of integrity and a reduction of intentions to switch. We distinguish consumer processing of analytical versus narrative response formats. Messages in analytical formats present a case by following a logical line of argument (Schellens \& de Jong, 2004). In contrast, narrative formats are essentially stories consisting of story characters that experience causally connected events within a particular context and time span (Brewer \& Lichtenstein, 1981). In the case of an analytical format, consumers tend to scrutinize response content, whereas a narrative format generally causes them to feel compelled. This engrossing effect commonly is referred to as transportation, which is conceptualized as an integrative melding of reader, story, and medium, focused on story characters and events (Chapter 2, p. 14). As a result of transportation, consumers are no longer aware of 
their prior beliefs, so a negative cognitive response is inhibited (Escalas, 2007; Slater, 2002b). Instead, they will empathize with the main character in a story (Green \& Brock, 2002). We argue that a reader of a narrative response to an integrity violation may thus come to empathize with the accused party. Consequently, empathic appeals should strengthen the inherent expression of regret in apologetic content. Conversely, such an effect is less likely when the reader processes a denial.

Second, to further our understanding of transportation in relation to narrative responses to blog posts, we restrict ourselves, from Study 2 on, to narrative formats and examine two recently identified transportation drivers: empathic and imaginative appeals (Green \& Brock, 2002). To date, research has yet to disentangle the potentially divergent impact of these drivers (Green \& Brock, 2000); we assess how the use of empathy and imagery may account for variation in levels of transportation in relation to responses to online integrity violations. Specifically, we examine whether consumers perceive different integrity levels when they empathize with the accused party or imagine events and thereby engage in transportation. This investigation may answer Singhal and Rogers (2002) call for a more comprehensive understanding of narrative processing and, therefore, transportation's unique effects on beliefs and intentions.

Third, we examine the narrative format of blog responses in relation to the other unique characteristic of blog posts, namely, the perspective of a specific narrator. Telling a story from the point of view of the person directly involved enhances the probability that readers will empathize with this person and his or her world view (Winterbottom, et 
al., 2008). We extend this concept and examine in Study 3 whether a personal response by the employee who is directly responsible for the integrity violation is more effective in restoring perceived integrity than is a response issued by the company's spokesperson. That is, we examine the impact of an important contingency on responses to integrity violations in consumer blogs (for an overview, see Figure 4-1).

\section{Conceptual background}

\section{Integrity restoration}

According to Hirschman (1970), consumers provide feedback to companies via two mechanisms, voice and exit. An allegation of violated integrity is an example of voice, whereas a switch to the competition is an exit. Integrity-based trust is negatively linked to the propensity to exit ( $R$. M. Morgan \& Hunt, 1994). Before describing why and how responses to integrity violations can restore integrity perceptions and reduce consumer exit, we must understand the nature of integritybased trust. In online marketplaces, various entities may be the objects of trusting beliefs and loyal intentions, including communities of vendors and users or the communication medium itself (Urban, Amyx, \& Lorenzon, 2009). In this sense, trust is based on integrity, that is, an entity is perceived to adhere to necessary or acceptable principles and standards (R. C. Mayer \& Davis, 1999). Previous trust research insufficiently addresses integrity, despite its increasing economic relevance during recent crises in markets as diverse as financial services, healthcare, telecommunications, and transport, which have suffered massive breakdowns in credibility (e.g., Plender, 2009). 


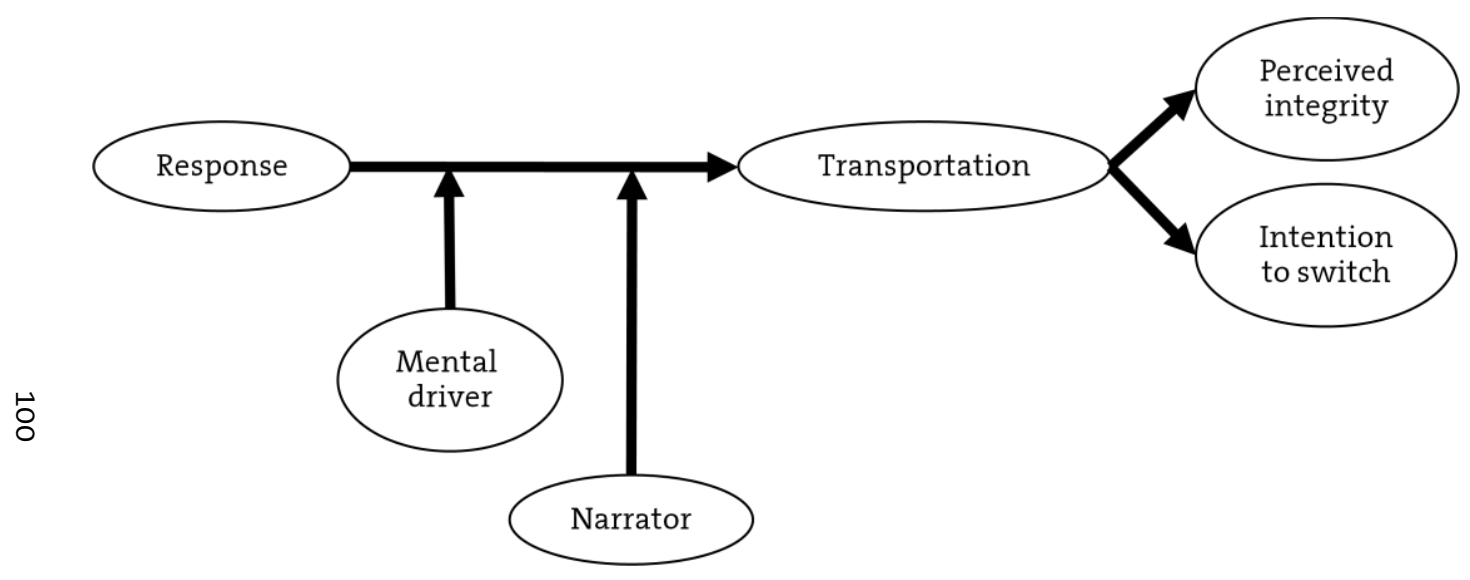

Figure 4-1

Conceptual model. 
The effects of integrity violations on beliefs include substantial decreases in the accused party's perceived trustworthiness compared with that of competitors, resulting in severe competitive vulnerability (Lewicki \& Bunker, 1996). Moreover, the very existence of the company may be threatened if consumers pursue an exit strategy in response to an integrity violation (Fornell \& Wernerfelt, 1987; Johnson \& Auh, 1998), even if an allegation is unsubstantiated (Kim, et al., 2004).

Verbal responses can restore integrity even before remedying behaviour can be displayed (Xie \& Peng, 2009). A restoration effort's acceptability results from elaboration of the company's culpability and from the likelihood that confidence may be breached again (Snyder \& Stukas Jr., 1999). In response to a violation, admitting wrongdoing signals guilt; a promise that the failure will not happen again implies redemption. Dual-process models of belief change (e.g., the Elaboration Likelihood Model, Petty \& Cacioppo, 1986) predict that consumers weigh the promise to behave well in relation to an admission of guilt. However, Snyder and Stukas Jr. (1999) show that in the case of an integrity violation, consumers attach more importance to guilt than to redemption signals. The rationale behind this finding is that consumers consider a lack of principles or awareness of moral consequences difficult to change.

Two common verbal responses are apology and denial (Lewicki \& Bunker, 1996). An apology relates positively to guilt. In contrast, a denial fails to signal guilt and addresses neither the details of the accusation nor the relevance of the domain (Snyder \& Stukas Jr., 1999). Rejecting culpability or attempting to counter the negative information may lead consumers to give the accused party the 
benefit of the doubt, sometimes even in the wake of contradictory evidence (Ditto \& Lopez, 1992). Overall, then, empirical evidence suggests that denials work best in response to an integrity violation (Ferrin, et al., 2007; Kim, et al., 2004).

\section{Hypotheses development}

We posit that the aforementioned relationships between integrity violations and responses hold primarily for an analytical processing pattern, as described in the Elaboration Likelihood Model. However, guilt signals may be less likely to have weight when people process narratives. Instead, such processing entails a dramatic view of the information, such that transportation may be the underlying mechanism (Escalas, 2004a, 2007; Green \& Brock, 2000, 2002; Slater \& Rouner, 2002).

Gerrig (1993) uses the term "transportation" to describe the feeling of entering the world evoked by the narrative. As a result of being transported, readers are no longer aware of their beliefs prior to reading because they are engrossed in the narrative events. When confronted with a claim that counters their intentions, consumers are inclined to draw on their prior beliefs to generate negative cognitive responses (Petty, Cacioppo, \& Schumann, 1983). In that case, an integrity restoration attempt will most probably fail. However, transportation inhibits such negative cognitive responses (Green \& Brock, 2000). Consequently, it "may lead to at least temporary acceptance of values and beliefs that represent a shift from the individual's existing beliefs" (Slater \& Rouner, 2002, p. 177). In other words, whereas readers tend to argue against analytical persuasive messages that are inconsistent with their prior beliefs and intentions, they do not do so when confronted with a narrative-based claim, even 
when it runs counter to their beliefs (Slater \& Rouner, 1996). Instead, transported readers empathize with the main story character (Green \& Brock, 2002). These transporting appeals may prompt readers of a narrative response to empathize with the accused party, strengthening the expression of regret. In this respect, apologies should evoke more empathy than denials because of their inherent capacity to move readers (Menon \& Dubé, 2007). Hence, we hypothesize that ceteris paribus:

\section{$\mathbf{H}_{1 \mathbf{a}}$ : When an accused party responds using an analytical format, consumers perceive greater integrity in that party if the response content is a denial rather than an apology. \\ $\mathbf{H}_{\mathbf{1 b}}$ : When an accused party responds using a narrative format, consumers perceive greater integrity in that party if the response content is an apology rather than a denial.}

Online customers can switch to the competition at virtually no cost, increasing the likelihood that in the case of a wrong response, companies accused of a failure of integrity incur damage in terms of customer exit. In that respect, an integrity failure is a source of competitive vulnerability (Lewicki \& Bunker, 1996). Yet Jaworski and Kohli (1993) demonstrate that when companies are responsive to their customers, the vulnerability is lessened. This implies that customers may still attach more importance to integrity-restoring consumercompany communications than to alternative service options and that such communications decrease the number who switch (de Ruyter \& 
Brack, 1993). Therefore, we hypothesize that ceteris paribus an analytical denial or a narrative apology by the accused company should evoke enough integrity to make consumers resist the temptation to exit.

$\mathbf{H}_{\mathbf{2 a}}$ : When an accused party responds using an analytical format, consumers switch less often if the response content is a denial rather than an apology.

$\mathbf{H}_{2 b}$ : When an accused party responds using a narrative format, consumers switch less often if the response content is an apology rather than a denial.

\section{Study 1}

\section{Method}

In Study 1, we examine the differential effect on perceived integrity and intention to switch of an analytical versus a narrative format in various response contents. Participants read three online texts: blog posts of an initial service encounter and integrity violation (both written by the same consumer) and a response by the service provider. The response was an analytical apology, an analytical denial, a narrative apology, or a narrative denial. This created a 2 (response format: analytical or narrative) $\times 2$ (response content: apology or denial) factorial design. In addition, to test whether the consumer's accusation was sufficient to change participants' integrity perceptions and intentions to switch, we assigned two control groups to the consumer's blog posts only. All participants were randomly assigned to the different experimental and control groups. 
Participants

University students from a medium-sized Dutch university $(n=153)$ participated. Their average age was 23 years. A minority (3.9\%) had children; $82.3 \%$ of the childless participants considered it likely they would have them in the future. Somewhat more women $(68.6 \%)$ than men took part in the experiment.

\section{Manipulations}

We chose to set the scene in a healthcare context for two reasons. First, Green, Brock and Kaufman (2004) claim that a topic's popularity may be a signal of its capacity to transport readers. Healthcare involves death, disease, and power, three "absolute interests" (for the complete nine, see Schank, 1979, p. 281). Second, the privatization of many hospitals has caused the sudden need for these service providers to compete in the market place. As a result, the relationship with their customers has undergone dramatic changes (Simmons, Birchall, \& Prout, 2007). Increasing evidence supports the importance of doctor-patient communication to reduce consumer intentions to switch (Tanner, 2004).

In the initial blog post, a patient spoke highly of her obstetrician. In the subsequent post, she accused him of an integrity failure during a problematic childbirth. In the blog's comment section, the doctor responded with either an apology or a denial. Moreover, the two versions adopted either analytical or narrative formats. In the narrative apology condition, the doctor stated for example: "I simply did a bad job with this birth, and I apologize for that", whereas in the denial condition, the doctor stated: "I did a good job with this birth, and I have 
no reason to apologize for that" (see Appendices, pp. 210-214 for more scenario fragments).

\section{Measures}

To assess perceptions of the doctor's integrity, we asked participants to indicate, on four Likerttype items, the extent to which they believed that the doctor possessed certain traits (see Appendices, p. 226). Six other items were aligned with participants' intentions to switch (see Appendices, p. 224). These items came from Roger Mayer and Davis (1999).

The measurement of whether the participants were equally capable of experiencing narratives involved assessments of their homogeneity in terms of empathic ability (Davis, 1983) and imageproducing capacity (Betts, 1909; Sheehan, 1967, see Appendices, pp. 222 and 224). The image-producing capacity scales ranged from "no image present at all, I only know that I am thinking of the object" to "perfectly clear and as vivid as the actual experience."

\section{Results}

Manipulation checks

For our confirmation of the response format manipulation (analytical or narrative), we asked participants to respond to a first manipulation check question about the features of the doctor's comment, followed by seven semantic scales (e.g., "arguments-images" and "a line of logic-a chain of events"; $\alpha=.91)$. The second question revealed whether the participants recognized the response content: "In the blog, Dr. Jacobs was accused of incorrectly delivering a baby. What was Dr. Jacobs' 
response to the accusation?" Their possible responses were: "He admitted to incorrectly delivering the baby and admitted personal responsibility for the problem.", "He did not admit to or deny the accusation.", or "He denied the accusation completely."

Responses to these two checks revealed that the manipulations were successful: Participants' format perceptions differed significantly between the analytical $(M=2.81, \mathrm{SD}=.77)$ and narrative response formats $\left(M=5.11, \mathrm{SD}=.54 ; t_{(99)}=16.60, p<.001\right)$, and all 101 participants not assigned to the control conditions answered the response content question correctly $\left.\alpha_{(2)}^{2}=73.11, p<.001, \varphi=.85\right)$. In Table $4-1$, we provide the average scores, reliabilities, and intercorrelations of the dependent and control variables.

\section{Table 4-1}

Study 1: Descriptive statistics.

\begin{tabular}{llllll}
\hline & & $M(\mathrm{SD})$ & $\alpha$ & 1 & 2 \\
\hline 1 & Intention to switch & $4.04(1.11)$ & .88 & & \\
& Perceived integrity & $3.47(1.19)$ & .83 & $-.60^{* *}$ & \\
2 & Empathic ability & $4.93(.97)$ & .82 & & \\
& Image-producing capacity & $5.35(.76)$ & .74 & & $.21^{* *}$ \\
\hline${ }^{* *} p<.01$. & & & &
\end{tabular}

Finally, our MANOVA for homogeneity indicates that the randomization was successful. Participants across all conditions were equally capable of experiencing narratives (Wilks' $\lambda=.96$, $\left.F_{(10,292)}=.67, p=.752\right)$. 


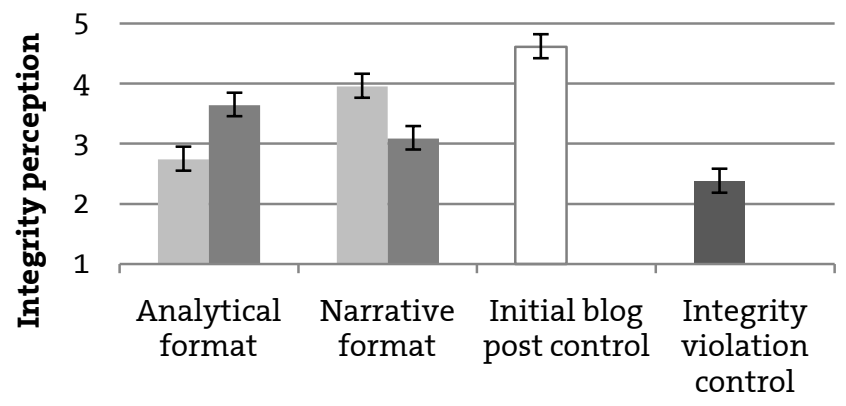

Apology $\square$ Denial

\section{Figure 4-2}

Perceived integrity for different response content and format combinations.

The error bars show the standard error.

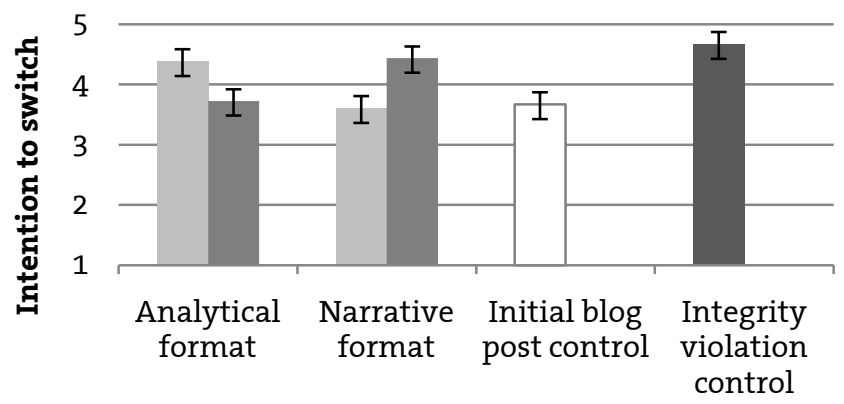

Apology $\backsim$ Denial

\section{Figure 4-3}

Intention to switch for different response content and format combinations.

The error bars show the standard error. 
Hypotheses tests

In Figures 4-2 and 4-3, we present the average scores and standard errors of perceived integrity and intention to switch across the analytical and narrative response formats, as well as the apology and denial response content conditions.

First, we conduct a $2 \times 2$ MANOVA for perceived integrity and intention to switch across the response content and format conditions. As predicted, we find a significant interaction effect (Wilks' $\lambda=.82, F_{(2,96)}=10.50, p<.001, \eta^{2}=.179$ ). To further test the hypotheses from $\mathrm{H}_{1 \mathrm{a}}$ to $\mathrm{H}_{2 \mathrm{~b}}$, we determine whether participants have a certain integrity perception and intention to switch as a result of reading the first blog post and whether the analytical denial and narrative apology may restore these. We also check whether the opposing response content and format combinations (e.g., analytical apology and narrative denial) do not soften the integrity violation. The two control groups did not read the doctor's response; specifically, 32 participants only read the initial blog post, and 20 participants read the initial post and the violation. A MANOVA across these groups and the response content and format conditions reveals a significant difference (Wilks' $\lambda=.56, \quad F_{(10,292)}=9.91, \quad p<.001$, $\eta^{2}=.253$ ). The univariate results also support $\mathrm{H}_{1 \mathrm{a}}, \mathrm{H}_{1 \mathrm{~b}}$, $\mathrm{H}_{2 \mathrm{a}}$, and $\mathrm{H}_{2 \mathrm{~b}}$ : the interaction effects on integrity perceptions $\left(F_{(5,147)}=20.92, \quad p<.001, \eta^{2}=.416\right)$ and intentions to switch $\left(F_{(5,147)}=4.69, p<.01, \eta^{2}=.138\right)$ are both significant.

We now report the simple contrasts. Participants, who reported their integrity perceptions after the initial post, the analytical denial, or the narrative apology, reported significantly higher integrity levels than those who reported their integrity perceptions after the 
violation, the analytical apology, or the narrative denial. $^{6}$ The differences of initial post versus analytical denial (mean difference=.97, $\mathrm{SE}=.23$, $p<.001$ ) and narrative apology (mean difference $=.66$, $\mathrm{SE}=.26, p<.05)$, as well as narrative denial versus violation (mean difference $=.71, \mathrm{SE}=.29, p<.05$ ), are also significant. The differences of analytical denial versus narrative apology, narrative denial versus analytical apology or violation, and analytical apology versus violation are not significant (mean differences<.40, $p s>.15$ ).

In terms of their softening effects on intentions to switch, the initial post, analytical denial, and narrative apology were also superior to the violation and to the opposing response content and format combinations. ${ }^{7}$

${ }^{6}$ initial post versus analytical apology: mean difference $=1.87$, $\mathrm{SE}=.24, p<.001$; initial post versus narrative denial: mean difference $=1.53, \mathrm{SE}=.26, p<.001$; initial post versus violation: mean difference $=2.23, \mathrm{SE}=.26, p<.001$; analytical denial vs. analytical apology: mean difference=.90, SE=.24, $p<.001$; analytical denial vs. narrative denial: mean difference $=.55$, $\mathrm{SE}=.26, \quad p<.05$; analytical denial versus violation: mean difference=1.26, $\mathrm{SE}=.27, \quad p<.001$; narrative apology versus narrative denial: mean difference $=.87, \mathrm{SE}=.29, p<.01$; narrative apology versus analytical apology: mean difference $=1.21$, $\mathrm{SE}=.27, p<.001$; narrative apology versus violation: mean difference $=1.58, \mathrm{SE}=.29, p<.001$

7 initial post versus analytical apology: mean difference $=.71$, $\mathrm{SE}=.27, \quad p<.05$; initial post versus narrative denial: mean difference $=.77, \mathrm{SE}=.30, p<.05$; initial post versus violation: mean difference=1.00, SE=.30, $p<.01$; analytical denial versus analytical apology: mean difference=.66, $\mathrm{SE}=.27, \quad p<.05$; analytical denial versus narrative denial: mean difference $=.71$, $\mathrm{SE}=.30, \quad p<.05$; analytical denial versus violation: mean difference $=.94, \mathrm{SE}=.30, p<.01$; narrative apology versus narrative denial: mean difference $=.83, \mathrm{SE}=.33, p<.05$; narrative apology versus analytical apology: mean difference $=.78, \mathrm{SE}=.30, p<.05$; narrative apology versus violation: mean difference $=1.06$, $\mathrm{SE}=.33, p<.01$ 
The remaining pairs do not differ significantly (mean differences<.30, $p s>.35$ ).

\section{Discussion}

Most prior empirical research considers whether narratives change beliefs (see Slater, 2002a). The results of Study 1 support our contention that narrative processing differs conceptually from analytical processing. In particular, an analytical denial and a narrative apology restore integrity perceptions and reduce intentions to switch more effectively than do their diametrically opposed response content and format combinations. Whereas Ferrin, Kim, and colleagues (2007; 2004) find that an analytical denial restores integrity perceptions because the response conveys a lack of guilt, we believe that a narrative apology's restoration effect results from the high level of transportation into the narrative world evoked by this combination, in response to which people do not engage in analytical elaboration (Slater \& Rouner, 2002). Regarding the competitive impact of narrative blog posts, transportation appears crucial for integrity perceptions to lead to a decrease in intentions to switch. Our analysis of narrative responses seems to support this claim. However, the data cannot be decisive with respect to the predicted causal relation between transportation and narrative-based integrity perceptions because we did not measure or manipulate transportation. Therefore, we test the narrative processingintegrity restoration relationship further by, in a second study, assessing and exploring what triggers transportation and its consecutive effects on narrative-based beliefs.

The analytical format is not considered from Study 2 on because consumers create blog posts to 
make sense of who they are (Herring, Scheidt, Bonus, \& Wright, 2004) and these communications are typically structured as a story (Scott, 1993). Consumers also complain about companies to friends online (Ward \& Ostrom, 2006). Delgadillo and Escalas (2004) show that such integrity violations are also in a narrative format. Furthermore, we find in Study 1 that within the "wrong" response content and format combinations, narrative denial restores integrity relatively more effectively (i.e., less badly) than does analytical apology or not responding at all. This finding echoes those of prior research that show a response format identical to that of the preceding communication is more persuasive than a different format (Adaval \& Wyer Jr., 1998), because information processes employed in one format may linger in a subsequent one (Schwarz \& Wyer Jr., 1985; Smith, 1990). Following this empirical evidence, we concentrate only on narrative formats because a narrative violation is best followed by a response in the same format.

\section{Study 2}

Green and Brock (2002) note two key transportation drivers: empathy and imagination. Empathy with the main character may explain the relationship between, on the one hand, transportation and, on the other, narrative-based changes in belief and intention. Readers may empathize with the main character by experiencing the narrative events. As Guber (2007, p. 56) suggests, "the spirit that motivates most great storytellers is: 'I want you to feel what I feel.'" Readers also may vividly imagine the narrative events. As a result, readers may be transported into the world of a 
narrative blog post, prompting them to believe that the (lack of) integrity they perceive is based on their mentally simulated experience (Feiereisen, Wong, \& Broderick, 2008). Thus, by priming the individual transportation drivers, we can further explore the particular effects of narrative responses to integrity violations.

In their experiments, Green and Brock (2000) report significant positive correlations between the level of transportation and liking the main story characters. However, although their research supports the claim that transportation can be manipulated successfully, they cannot disentangle empirically the ways in which transportation changes beliefs. Therefore, in Study 2, we examine whether independent mental priming of empathy and imagery influences perceived integrity, mediated by transportation. In summary, when the accused party responds with a narrative apology, we hypothesize that ceteris paribus

$\mathbf{H}_{3}$ : Consumers exhibit greater beliefs in the integrity of an accused party if they engage with empathy rather than imagery.

$\mathbf{H}_{4}$ : $\quad$ Transportation mediates the relationship between empathy and perceived integrity.

\section{Method}

Using a procedure similar to that of Study 1, we asked participants to read an online text. We told them that this was a blog post featuring a resignation speech by a $\mathrm{CEO}$ of a public railway company in a Western European country, posted in the wake of a corruption scandal that had broken two days earlier, in which he was accused of 
transferring public funds to his private account. For the sake of generalizability, we used such an announcement instead of an integrity-violating blog post, thereby preventing possible confounding effects of empathy with the accusing party, not just the accused.

In our manipulations of the response content, the CEO either apologized for what he did or denied the accusation. We also gave distinct sets of instructions about how to approach the reading task, priming one group to empathize with the CEO and the second group to imagine the events that took place. The control group was not instructed to process the narrative in any particular way. The participants were randomly assigned to the response content and priming conditions. The study thus used a 2 (response content: apology or denial) $x$ 3 (priming: empathy, imagery, or control) factorial design.

\section{Participants}

Of the 145 students who participated, 52.4\% were women and $87.6 \%$ travelled by train at least once a month. The participants' average age was 23 years.

\section{Manipulation and priming}

To test the relationship between the response content and the transportation drivers, we created two narrative blog posts: an apology and a denial. For example, the following excerpt comes from the narrative apology: "To every train passenger-and to all those who believed in what I tried to stand for-the accusation is true. I am guilty of transferring funds to my private account, and I sincerely apologize." Participants in the denial 
condition read: "To every train passenger-and to all those who believed in what I tried to stand forthe accusation is false. I am not guilty of transferring funds to my private account. I deny everything I was accused of over the last few days" (see Appendices, pp. 214-216 for more scenario fragments).

To distinguish between the levels of transportation, we used three primings: empathy, imagery, and control. Our empathy condition follows the method used by Green and Brock (2000). It attempted to encourage participants to feel empathy for Adam Klein, the CEO. After the introduction to the study, participants read from a computer screen:

The text of the blog post will be used as a voiceover in a movie on the events leading to Adam Klein's resignation. Your responses will help the actor playing Adam Klein to become involved in his role. While reading this blog post, use your emotion. Think about how Adam Klein was feeling and how you might feel in the situation. Place yourself in Adam Klein's shoes. [...] You are now Adam Klein!

The imagery priming instructions encouraged participants to picture the narrative events. The instructions noted:

The text of the blog post will be used as a voiceover in a movie on the events leading to Adam Klein's resignation. Your responses will help the director of the movie to select the most important events. While reading this blog post, use your imagination. Think about the settings and what happened. Form a helicopter view of Adam Klein's life. [...] You are now a movie director!

The control group simply read the blog post. 


\section{Measures}

To measure transportation effects, we used the items developed by Green and Brock (2000). The Likert-type scales ranged from "strongly disagree" to "strongly agree." We adapted the items' formulations slightly to make them appropriate for responses to the blog post (see Appendices, p. 228).

Except for a slight adaptation to the particular context, the perceived integrity measures were identical to those used in Study 1.

As in Study 1, we assessed the participants' homogeneity. Because the priming instructions could interact with empathic or imaginative ability measures, we compared the participants in the various conditions according to Tellegen and Atkinson's (1974) absorption scale, which measures the general tendency to become immersed in life experiences (see Appendices, p. 221).

\section{Results}

Manipulation and priming checks

To check the content manipulation, we used a question similar to that in Study 1, adapted to the CEO situation. Only two participants misidentified either the apology or denial $\chi_{(2)}^{2}=126.32, p<.001$, $\varphi=.93)$.

A question from Green and Brock (2000) also measured the participants' understanding of their task while they were reading the blog post. They answered on two semantic scales anchored by "use my emotion-use my imagination" and "think about how Adam Klein is feeling, and how I might feel in the situation-think about the settings and what happened" $(\rho=.68, p<.001)$. The item averages differentiate reliably between the priming 
conditions $\left(t_{(98)}=8.32, p<.001\right)$, such that the empathy-primed participants tried to empathize with the CEO $(M=2.76, S D=1.55)$ and the imageryprimed participants attempted to picture the narrative events $(M=5.24, \quad S D=1.42)$. Thus, the manipulation and priming were successful. In Table 4-2, we list the average scores, reliabilities, and intercorrelations of the variables.

\section{Table 4-2}

Study 2: Descriptive statistics.

\begin{tabular}{lllll}
\hline & & $M(\mathrm{SD})$ & $\alpha$ & 1 \\
\hline 1 & Perceived integrity & $3.58(.92)$ & .84 & \\
& Transportation & $3.90(.79)$ & .74 & $.17^{*}$ \\
& Absorption & $4.37(.88)$ & .73 & \\
\hline${ }^{*} p<.05$. & & &
\end{tabular}

We find no differences for absorption between the conditions. All groups show the same tendency to engage in life experiences $\left(F_{(5,139)}=1.80, p=.117\right)$. This indicates that the randomization was also successful.

\section{Hypotheses tests}

We present the average scores and standard deviations of perceived integrity and transportation across the apologizing and denying response content and the empathy, imagery, and control conditions in Table 4-3. 


\section{Table 4-3}

Study 2: Perceived integrity and transportation as functions of response content and priming instruction.

\begin{tabular}{llll}
\hline Content & Priming & $\begin{array}{l}\text { Perceived integrity } \\
M(\mathrm{SD})\end{array}$ & $\begin{array}{l}\text { Transportation } \\
M(\mathrm{SD})\end{array}$ \\
\hline Apology & Empathy & $4.14(1.02)$ & $4.30(.67)$ \\
& Imagery & $2.97(.94)$ & $3.72(.62)$ \\
& Control & $3.69(.66)$ & $4.02(.83)$ \\
Denial & Empathy & $3.59(.76)$ & $4.17(.81)$ \\
& Imagery & $3.53(.89)$ & $3.89(.60)$ \\
& Control & $3.53(.84)$ & $3.25(.78)$ \\
\hline
\end{tabular}

A $2 \times 3$ analysis of variance to test $\mathrm{H}_{3}$ and $\mathrm{H}_{4}$ reveals significant differences between priming conditions' effects on restored integrity $\left(F_{(2,139)}=6.26, p<.01\right.$, $\left.\eta^{2}=.083\right)$. Specifically, participants primed to empathize with the CEO perceive more integrity than those who pictured the narrative events (mean difference=.63, SE=.18, $p<.01)$. These priming conditions do not differ significantly from the control (mean differences<.40, ps>.05).

The ANOVA also reveals an interaction effect of priming and response content on restored integrity $\left(F_{(2,139)}=5.20, p<.01, \eta^{2}=.070\right)$. Simple contrasts show that the apology restores perceptions of integrity more effectively with empathy than with imagery priming (mean difference=.55, SE=.24, $p<.05$ ). The empathy and control conditions differ marginally (mean difference $=.45, \mathrm{SE}=.25, p=.079$ ). However, the empathy, imagery, and control conditions do not provoke different integrity perceptions after the denial (mean differences $<.06, p s>.80$ ). These results provide strong support for $\mathrm{H}_{3}$. 
The instructions create significantly different transportation effects $\left(F_{(1,95)}=14.57, p<.001, \eta^{2}=.113\right)$. Participants instructed to empathize with the CEO indicated that they felt more transported into the response than did those who were told to invoke their imagination (mean difference=.63, $\mathrm{SE}=.15$, $p<.001)$. We find no significant differences between the imagery priming and control groups (mean difference $=.20, \mathrm{SE}=.16, p=.212$ ).

To test $\mathrm{H}_{4}$, we also conduct a mediation analysis and compare participants who are primed to empathize with the CEO with the control group across both apology and denial conditions. The empathy-primed participants perceive the most integrity $\left(F_{(3,95)}=2.79, \quad p<.05, \eta^{2}=.082\right)$. When we include transportation in the equation as a covariate, the former effect becomes insignificant $\left(F_{(3,92)}=2.22, p=.091\right)$. A subsequent Sobel test derived from MacKinnon and colleagues (2002) and Zhao and colleagues (2010) offers strong support for $\mathrm{H}_{4}$ $(Z=1.84, p<.05)$.

\section{Discussion}

Our results clearly demonstrate that the use of empathy restores perceptions of the integrity of the accused party more effectively than does imagery. Empathy also transports readers much further into the narrative response than does imagery. Furthermore, narrative apology readers appear to display stronger integrity perceptions as a result of empathizing with the accused party, rather than as a result of imagining the events described in the narrative. Another important finding indicates that the impact of empathy on integrity perceptions is mediated by transportation. For a narrative apology, empathy seems to provide a crucial driver for provoking transportation. The apparent significance 
of empathy prompts us to examine further how it might be evoked most effectively. In our next study, we relate to different narrators.

\section{Study 3}

In our first two studies, the person responsible for the service failure responded to the accusation. In reality, however, a company's spokesperson or public relations (PR) professional commonly formulates a reply, presumably to exploit the (in comparison to the accused person) greater credibility of the PR spokesperson. However, in narrative processing, source credibility may be largely irrelevant. Instead, empathy with the persons portrayed in the narrative appears to determine transportation into and thus the impact of the narrative content (Slater, 1997). In turn, blog post readers may experience more empathy toward the involved employee than toward a PR spokesperson, who may seem more aloof, associated with "cheap talk" (Farrell \& Rabin, 1996), and not in a position to remedy the specific integrity violation. In Study 3, we test this largely overlooked incongruity effect between the narrator and message content. Ceteris paribus,

\footnotetext{
$\mathbf{H}_{5}: \quad$ Consumers perceive greater integrity in the accused party if the responsible employee, rather than the company's spokesperson, issues the apology.
}

\section{Method}

Our previous experiments took place in a healthcare or public services context-markets in which competition is still relatively low (though 
this characteristic is changing as a result of widespread privatization). To confirm the basic findings of Studies 1 and 2 in a highly competitive market, we selected a classic retailing case: a dishonest car dealer, called R\&W Cars. As in Study 2, participants first read an announcement of the dealer's behaviour and then read a blog post with a narrative apology. We manipulated the perspective taken in the narrative to determine empathy (Winterbottom, et al., 2008). Participants were randomly assigned to these high or low empathy conditions in the between-subjects design.

Participants

For the study, 95 students were invited to participate. Women constituted $50 \%$ of the participants. The participants were 22 years of age on average. While $30.5 \%$ owned a car, $56.4 \%$ of the carless participants considered it likely that they would buy one in the future.

\section{Manipulation}

We used two versions of the same narrative apology: In the high empathy condition, the apology came from the responsible sales representative. He stated: "Please allow me to present my side of the story so that I can, I hope, make you understand." In the low empathy condition, the apology came from a distant spokesperson. He said: "We would like to take a moment to present our side of the story for your information" (see Appendices, pp. 216-217 for more scenario fragments). 


\section{Measures}

The dependent and control measures are largely similar to those in Study 2, namely, the extents to which the participants experience transportation, perceive integrity, and tend to become absorbed in life experiences.

\section{Results}

Manipulation checks

First, to assess the degree of empathy, we slightly adapted the formulation of Davis's (1983) empathic ability items to make them appropriate to the response to the blog post context; examples include "While I was reading the blog post, I imagined how I would feel if the events in the story were happening to me" and "I did not get extremely involved in the blog post" (reverse coding) ( $\alpha=.83$ ). The manipulation was successful because participants in the high empathy condition report significantly more empathy $(M=4.67, \mathrm{SD}=.89)$ than those in the low empathy condition $(M=3.47$, $\left.\mathrm{SD}=1.04 ; t_{(93)}=5.88, p<.001\right)$. We offer, in Table $4-4$, the average scores, reliabilities, and intercorrelations of the dependent and control variables.

\section{Table 4-4}

Study 3: Descriptive statistics.

\begin{tabular}{lllll}
\hline & & $M(\mathrm{SD})$ & $\alpha$ & 1 \\
\hline 1 & Perceived integrity & $4.19(1.11)$ & .89 & \\
& Transportation & $4.01(.65)$ & .79 & $.21^{*}$ \\
& Absorption & $4.37(.83)$ & .71 & \\
\hline
\end{tabular}

${ }^{*} p<.05$. 
The level of absorption does not differ as a function of condition $\left(t_{(93)}=1.73, \quad p=.086\right)$, so our randomization was successful.

Hypotheses tests

We present the average scores and standard deviations of perceived integrity and transportation across the high and low empathy conditions in Table 4-5.

\section{Table 4-5}

Study 3: Perceived integrity and transportation as functions of response content and priming instruction.

\begin{tabular}{lll}
\hline Empathy & $\begin{array}{l}\text { Perceived integrity } \\
\end{array}$ & $\begin{array}{l}\text { Transportation } \\
M(\mathrm{SD})\end{array}$ \\
\hline High & $4.40(1.04)$ & $4.23(.52)$ \\
Low & $3.82(1.15)$ & $3.62(.69)$ \\
\hline
\end{tabular}

We conduct an ANOVA with perceived integrity as the dependent variable and empathy as a betweensubject factor. Empathy has a significant effect on perceived integrity $\left(F_{(1,93)}=6.15, p<.05, \eta^{2}=.062\right)$. Those manipulated to sense higher empathy exhibit more integrity perceptions, on average, than those in the low empathy condition. Thus, $\mathrm{H}_{5}$ receives support.

As a second test of $\mathrm{H}_{4}$, pertaining to the mediating effect of transportation, we include the level of transportation as a covariate in the ANOVA for perceived integrity. The empathy effect becomes insignificant $\left(F_{(1,92)}=2.99, p=.087\right)$. A Sobel test derived from MacKinnon and colleagues (2002) and Zhao and colleagues (2010) indicates that the effect of empathy on integrity perception is mediated 
significantly by the level of transportation $(Z=1.90$, $p<.05)$.

\section{Discussion}

In Study 3's extension of contemporary research, we examine the effects of the perspectives adopted by the narratives (Winterbottom, et al., 2008). Our results reveal that the point of view in a narrative blog post significantly explains empathy with the narrator, which affects perceived integrity. In other words, consumers confronted with an apology by the responsible violator exhibit more empathy and integrity perceptions than those confronted with the company's spokesperson. As expected, this linkage remains robust in markets characterized by high levels of competitive intensity. A narrative offered by the focal employee produces such empathy-is so transporting-that consumers restore integrity perceptions, despite content that blatantly mentions an alternative option for service. Consumers who feel empathy for the responsible employee become transported into his or her narrative and therefore have fewer cognitive resources available to draw on their prior beliefs and interpret what the high competitive intensity is suggesting.

\section{Limitations}

Even though we investigate the moderating effect of the perspective taken in the company response, we fail to incorporate some other moderating variables, such as loyalty toward the company or brand. Loyal customers may be naturally inclined to empathize with a brand as a result of their relationship tenure or breadth. They 
may experience more transportation when confronted with a narrative about that particular brand (Escalas, 2004b). Arguably, such consumers will agree with the covertly persuasive narrative as a matter of course.

Unlike loyal consumers, disgruntled consumers have no goal other than to spread rumours and bring down an accused company. Ward and Ostrom (2006) demonstrate that these investigative consumers quickly form ad hoc groups online. Consequently, although we show that both analytical denials and narrative apologies lead consumers to grant the accused party the benefit of the doubt, there may also be other forces at work in the blogosphere. As a result, companies may not always have the option of denial when they face an (illegitimate) accusation.

Finally, we have assumed that the accused companies automatically take responsibility for the consumer complaint. However, as the current crisis in financial markets illustrates, it may not be obvious that companies will take negative stories about their performance seriously (e.g., Chapter 3 and Plender, 2009).

In conclusion, the findings of this chapter confirm the positive relationship among empathy, transportation, beliefs and intentions. A light switch can illustrate Green and Brock's (2000) findings that transportation can be switched on; we extend the metaphor by introducing the notion of a dimmer switch, in that empathic appeals represent a brighter manifestation of the same light and may prevent consumers from switching off in the process. 



\section{5 \\ THE MEANS TO JUSTIFY THE END}

How narrative transportation affects justice of anti-harassment measures in social media

\section{Introduction}

The rapidly growing use of social media is dramatically influencing the way consumers share information and interact with companies. In particular, these virtual meeting and marketplaces support the development and maintenance of multiple forms of identity that can contribute to personal branding (Cheung \& Lee, 2010). For example, a profile page and wall of posts bear detailed witness to personal stories (Schau \& Gilly, 2003), and membership in company-sponsored fan and interest group pages reinforces collective identity (Muniz Jr. \& O'Guinn, 2001). Yet such networks run a seemingly increasing risk of exposing members to ridicule and insults (LaBrie, Collier, \& Palmer, 2010; The Economist, 2010). When consumers treat one another disrespectfully, the resulting cyber bullying may cause members to exit social networking sites, a trend that has affected Facebook as well as weblog communities run by Procter \& Gamble, Sony, and Walmart (Edelman, 2010; Martin \& Smith, 2008; Moore, 2009).

In response, companies attempt to implement structural measures that might counteract cyber harassment, such as by altering the privacy controls on user accounts to regulate access to personal 
information (Krasnova, et al., 2010; Sledgianowski \& Kulviwat, 2009). Paradoxically though, tightening the rules limits the freedom of self-expression and identity building undertaken by benevolent consumers (Kleinrock, 2004). Thus Facebook came under criticism recently for failing to tell members it was disabling a feature that shares user addresses and telephone numbers with external applications and websites (Bowen, 2011; Purdy, 2011). The social media community often regards such sudden and unexplained decisions by network providers as violations of their painstakingly crafted personal and social identities (Raphael, 2009; Walters, 2009) - an issue that provokes strong feelings and complaints about any restrictions on freedom of expression (Urban, et al., 2009). Therefore, network providers must determine how to address cyber harassment in a way that members regard as necessary, appropriate, and in their best interests instead of as an unjust practice.

Accordingly, theory in social psychology might be instructive, in that it makes a clear distinction between the personal and social components of identity (Dollinger, Preston, O'Brien, \& DiLalla, 1996; Reid \& Deaux, 1996; Skitka, 2003). Both are part of an active self-concept, but whereas social identity derives from membership in a group, personal identity refers to pursuits that set a person apart from referent others (Hewitt, 2003; Tajfel \& Turner, 2004). The two components also differ with respect to their influence on reactions to limitations, such that decisions made to limit cyber harassment should seem more distributively and procedurally just viewed from the perspective of a social identity violation (D. M. Mayer, Greenbaum, Kuenzi, \& Shteynberg, 2009). For a social identity, "sorrow shared is sorrow halved," such that enforced regulation is endured more easily when shared with 
others. Despite these contributions, David Mayer and colleagues focus solely on decisions that present a logical case for stricter behaviour regulations, including consecutive arguments, as they illustrate with their experimental stimulus (2009, p. 147):

To give you a brief background, there have been issues with the use of profanity in speech and on t-shirts, inappropriate cheers, and rioting after certain sporting events. The administration believes something must be done to address this problem. Recently, the administration met to determine how to handle this issue. The administration decided that [...].

It remains unclear however whether such an analytical, factual rationale for decisions (Schellens \& de Jong, 2004) leads to justice perceptions among social media users.

In line with the definition of social media as "web-based services that allow individuals to construct a public or semi-public profile within a bounded system" (Doyd \& Ellison, 2007, p. 211), two key characteristics of social media likely influence justice perceptions. First, users construct a profile by sharing stories about personal experiences, brand preferences, and significant (or less significant) events (Kaplan \& Haenlein, 2010). Thus Delgadillo and Escalas (2004) posit that on social exchange platforms, consumers are main characters in their own narratives, which gives social media messages a predominantly narrative format. Second, the public profile constructed through storytelling may diverge from users' private selves (Kozinets, et al., 2010; Schau \& Gilly, 2003). Virtually no research examines violations of the constructed public self, even though social network providers increasingly 
implement measures that may restrict members' self-expression.

Narratives often cause readers to become engrossed (Slater, 2002a), in an effect referred to as transportation, defined as an integrative melding of reader, story, and medium, focused on story characters and events (Chapter 2, p. 14). If network users read an engrossing narrative account of cyber harassment, they should be transported and put themselves in the victim's shoes. Thus, a case for privacy regulations might be justified more persuasively if based on a narrative account, relative to an account founded on facts or figures. In turn, the regulation may seem more just, regardless of whether it represents a personal or social identity violation. We examine for the first time the role of the format of cyber harassment reports on perceptions of the justice of privacy regulations.

In addition, to further understanding of privacy regulation acceptance in relation to this narrative format, we examine the influence of referencing (Escalas, 2007), that is, the attribution of story events to a particular identity (West, et al., 2004). In Study 1, we examine how readers of a transporting narrative take the perspective of a victimized fellow network member, and we argue that the more people relate reports of cyber harassment to their own (social media) identity, the more they become transported. In the case of social media, readers may then relate defamations to their own public profile or even their private selves. In Study 2, we also explore how diverging reference identities might affect transportation levels and thus perceptions of the justice of privacy regulation decisions. (for an overview, see Figure 5-1). 


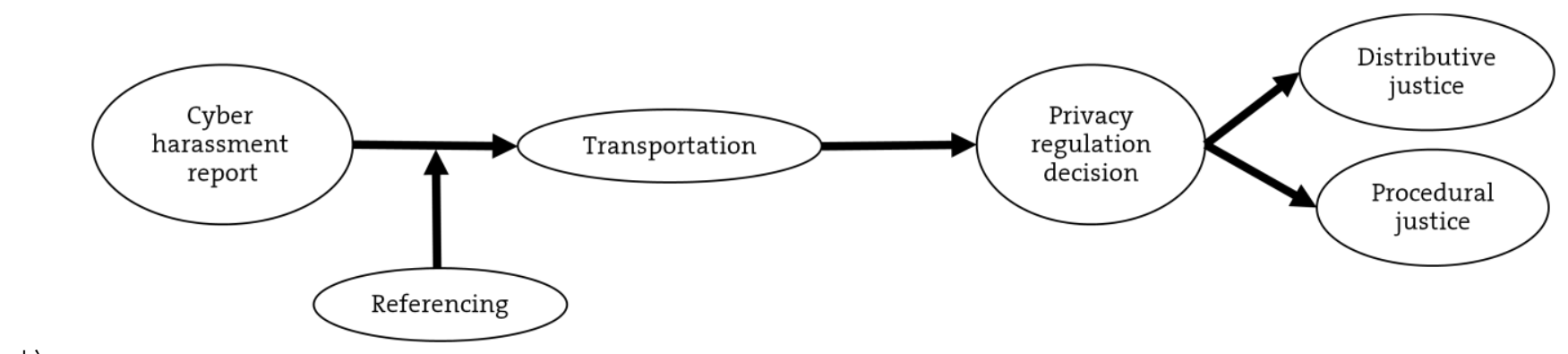

$\stackrel{\stackrel{\omega}{ }}{ }$

\section{Figure 5-1}

Conceptual model. 


\section{Conceptual background}

\section{Identity violation endurance}

Embarrassing or false information about social media users frequently gets posted on social networks (Pitcher, 2007). To deal with such cyber harassment, network providers subject consumers to restricted access to personal information, which aims to regulate privacy controls. Such decisions by providers have consequences for the justice perceptions of users. Justice perceptions traditionally are associated with beliefs about moral rightness, based on a sense of fairness (Rawls, 1971, 1999), whether distributive or procedural (CohenCharash \& Spector, 2001; Colquitt, Conlon, Wesson, Porter, \& Ng, 2001). Distributive justice results from decision outcomes that adhere to principles of equal allocation. Procedural justice is the result of a fair decision-making process. Whether people consider justice depends on the relative accessibility of fairness thoughts and the degree to which they are aware of their identity (Skitka, 2003). Research on justice reasoning thus highlights two main components of identity: personal and social (Dollinger, et al., 1996; Reid \& Deaux, 1996). Personal identity refers to "the person's construction and maintenance of an autobiography-a life story that is built, told to (and by) others in various contexts, and from time to time revised to fit changing experiences or preferences" (Hewitt, 2003, p. 111). In contrast, the social component of identity derives from membership in various groups (Tajfel \& Turner, 2004), including brand fan pages such as Apple's (>46 million members) or Mercedes-Benz's (>34 million members). Theoretical and empirical support indicates that people aspire to protect and 
uphold their personal and social identities (Baumeister \& Leary, 1995; Steele, 1988). In the case of an identity threat, the person becomes more aware of her or his identity. Violations then make thinking about fairness more salient. When an external decision violates a person's personal or social identity, it also should have a negative influence on justice perceptions.

David Mayer and colleagues (2009) note that people devalue justice less if a decision restricts the liberties of a group to which they belong. That is, identification with a group initiates a process of depersonalization, so members' conceptualization of themselves in that context relates more to group norms (Terry \& Hogg, 1996). Decisions that affect group members overall are more likely to prompt evaluations based on justice concerns for the group, rather than for the individual (Leung, Tong, \& Lind, 2007). Such evaluations may cause the resulting justice perceptions to be less negative than they would be if the restrictive decisions were personally damaging.

\section{Hypothesis development}

As we noted, identity research has shown that external decisions that pertain to people's social identity affect justice perceptions less than decisions relevant to their personal identity, because people consider a widespread restriction more just. However, these relationships may not hold for social media because of the largely narrative format of its messages. The phenomenon of transportation, or mentally entering a narrative, should play a crucial role in this case (Green \& Brock, 2002; Slater \& Rouner, 2002). Transportation underlies the processing of various narratives, featuring in print advertisements (Escalas, 2004b), 
TV commercials (Wang \& Calder, 2009), and social media messages (see this dissertation). It is adjacent to but conceptually different from mental states that involve feelings of immersion in challenging activities, such as optimal experience or flow (Csikszentmihalyi, 1992). Being transported into narrative accounts of cyber harassment also may facilitate acceptance of privacy regulation decisions by network providers. In particular, we posit that social media users feel like the storyteller or see narrative events through the eyes of the narrator (Escalas, et al., 2004; Green \& Brock, 2002). Such perspective taking should inhibit social media users from generating counterarguments (Slater \& Rouner, 2002). This inhibition also may produce the perception that privacy restrictions, presented in response to narratives about harassment online, are just. Specifically, the narrative format of reports of cyber harassment shared in social media should facilitate the acceptance of privacy regulation decisions, and we hypothesize

$\mathbf{H}_{\mathbf{1}}$ : When a privacy regulation decision is presented as a response to a narrative cyber harassment report, the identityviolating effect of the decision on (a) distributive and (b) procedural justice perceptions decreases compared with a decision presented in response to an analytical report. 


\section{Study 1}

\section{Method}

In Study 1, we examine the effect of privacy regulation on perceived distributive and procedural justice, according to various identity components and differently formatted cyber harassment reports. Participants read two online texts: a wall post about a cyber harassment experience (written by another network user) and a privacy regulation decision (written by the network provider). The wall post used either a narrative or an analytical format, and we measured whether the network provider's decision affected the personal and/or social components of the participants' identities. Thus we used a 2 (cyber harassment report: narrative or analytical format) $\times 2$ (personal identity: violated or not) $\times 2$ (social identity: violated or not) factorial design. All participants were randomly assigned to the different experimental groups.

\section{Participants}

University students from a medium-sized Dutch university $(n=124)$ participated in this study. Their average age was 25 years, 60 were women, and most (89\%) were members of a social networking site who spent an average of nine hours per week using social media.

\section{Manipulations}

In the first wall post, a fellow network member reported profanity in status updates and comments, inappropriate wall posts, and offensive pictures. The accounts adopted either a narrative or analytical 
format. In the narrative condition, the comment began as follows: "I'd like to tell you about a cyber harassment episode-how it has influenced me-by presenting my side of the story." In the analytical condition, the comment began as follows: "As you are aware, article 5 in the terms of use of the social network regards the issue of user behaviour on profile pages. It states that cyber harassment 'involves the use of the social network to support deliberate, repeated, and hostile behaviour by an individual or group of users that is intended to harm other users'." The rest of the post provided information about the decision the network provider made to restrict social network users' behaviour (see Appendices, pp. 217-219 for the wall post fragments). We used David Mayer and colleagues' (2009) personal and social identity violation measures to assess which identity component(s) the decision affected. The personal identity violation measure contained four Likerttype items, including "I feel like the decision made by the network provider violated some of my personal identity." Social identity violation similarly was measured with four Likert-type items, such as "I feel the decision made by the network provider is damaging to the social media users." In line with David Mayer and colleagues, we used two median splits to distinguish violations of personal and social identities and then entered these factors into the analysis as two 2-level variables, to assess their effect on justice perceptions.

\section{Measures}

To assess perceptions of distributive justice, we asked participants to indicate, on six Likert-type items, the extent to which they believed that the decision was just (see Appendices, p. 222). Colquitt 
(2001) and Gilliland (1994) have validated these scales. Three other items referred to participants' perceptions of procedural justice (see Appendices, p. 227), adopted from Brockner and colleagues (2005).

To measure transportation effects, we used the items that Green and Brock (2000) developed. The Likert-type scales ranged from "strongly disagree" to "strongly agree." We adapted the items' formulations slightly to make them more appropriate as responses to the cyber harassment report (see Appendices, p. 228).

To assess the effects of the report, we needed the different participant groups to be equally capable of processing narrative and analytical report formats. Therefore, we measured whether participants were homogeneous in this respect according to their absorption tendency (Tellegen \& Atkinson, 1974) and need for cognition (Cacioppo, Petty, Feinstein, \& Jarvis, 1996). Because a social identity violation requires some identification with the social group, we also measured social identification (D. M. Mayer, et al., 2009, see Appendices, pp. 221 and 226-227).

\section{Results}

Manipulation checks

We checked the manipulation (narrative or analytical format) by administering seven semantic scales that could describe the cyber harassment report format (e.g., "arguments-images," "a line of logic-a chain of events"; $\alpha=.96)$. A $t$-test shows that the participants successfully distinguished between a narrative $(M=5.70, \mathrm{SD}=.38)$ and an analytical format $\left(M=2.26, \quad \mathrm{SD}=.34 ; \quad t_{(122)}=53.09, \quad p<.001\right)$. To clarify the manipulation, we also performed a $t$-test 
on the transportation measure, which revealed a significant difference $\left(t_{(122)}=15.46, p<.001\right)$. That is, participants experienced more transportation in the narrative $(M=5.05, \mathrm{SD}=.85)$ than in the analytical $(M=2.74, \quad \mathrm{SD}=.81)$ report format, and the manipulation was successful. We present the average scores, reliabilities, and intercorrelations of the dependent and control variables in Table 5-1.

\section{Table 5-1}

Study 1: Descriptive statistics.

\begin{tabular}{llllll}
\hline & & $M(\mathrm{SD})$ & $\alpha$ & 1 & 2 \\
\hline 1 & Distributive justice & $4.50(1.43)$ & .98 & & \\
2 & Procedural justice & $4.50(1.28)$ & .95 & $.52^{* *}$ & \\
& Transportation & $4.09(1.42)$ & .98 & $.25^{* *}$ & $.16^{*}$ \\
& Absorption & $3.86(1.18)$ & .95 & & \\
& Need for cognition & $4.44(1.10)$ & .97 & & \\
& Social identification & $4.02(1.14)$ & .86 & & \\
\hline${ }^{*} p<.05 ;^{* *} p<.01$. & & & &
\end{tabular}

To confirm the randomization, we performed a homogeneity check across all conditions pertaining to absorption, need for cognition, and social identification. A multivariate analysis of variance (MANOVA) showed that the conditions did not differ across control variables (Wilks' $\lambda=.97$, $\left.F_{(3,120)}=1.38, p=.250\right)$. Participants were equally capable of processing narrative and analytical report formats and identifying with their social group, regardless of their assigned condition. 
Hypothesis test

In Table 5-2, we present the average scores and standard deviations of perceived distributive and procedural justice across the analytical and narrative report formats, as well as the personal and social identity violation conditions.

\section{Table 5-2}

Study 1: Distributive justice and procedural justice as a function of cyber harassment report as well as personal and social identity violation.

\begin{tabular}{lllll}
\hline $\begin{array}{l}\text { Report } \\
\text { format }\end{array}$ & $\begin{array}{l}\text { Personal } \\
\text { identity }\end{array}$ & $\begin{array}{l}\text { Social } \\
\text { identity }\end{array}$ & $\begin{array}{l}\text { Distributive } \\
\text { justice } \\
M(S D)\end{array}$ & $\begin{array}{l}\text { Procedural } \\
\text { justice }\end{array}$ \\
\hline Narrative & Violated & Violated & $4.00(.88)$ & $4.40(1.01)$ \\
& Not & $4.40(1.00)$ & $4.55(.62)$ \\
& $\begin{array}{l}\text { Not } \\
\text { violated }\end{array}$ & $\begin{array}{l}\text { Violated } \\
\text { Not }\end{array}$ & $5.51(.83)$ & $5.43(.90)$ \\
& $\begin{array}{l}\text { violated } \\
\text { Analytical }\end{array}$ & $6.03(1.03)$ & $5.37(.74)$ \\
& Violated & Violated & $3.08(1.34)$ & $3.10(1.10)$ \\
& & $\begin{array}{l}\text { Not } \\
\text { violated }\end{array}$ & $3.52(1.07)$ & $3.50(.51)$ \\
& Not & Violated & $4.90(1.23)$ & $4.80(1.12)$ \\
& & $\begin{array}{l}\text { Not } \\
\text { violated }\end{array}$ & $5.00(1.12)$ & $4.96(1.11)$ \\
\hline
\end{tabular}

To test our hypothesis about the role of the cyber harassment reports, we investigated the effect of report format and identity violations on distributive and procedural justice perceptions. A $2 \times 2 \times 2$ MANOVA revealed a significant interaction effect of report format and personal identity violation (Wilks' $\lambda=.52, F_{(2,115)}=54.12, p<.001, \eta^{2}=.491$ ) and a main effect of report format (Wilks' $\lambda=.73$, 
$\left.F_{(2,115)}=20.73, p<.001, \eta^{2}=.274\right)$. The other effects were not significant at the .05 level. The univariate tests of distributive justice perceptions for the interaction between report format and personal identity violation $\left(F_{(1,117)}=66.15, p<.001, \eta^{2}=.322\right)$ and the main effect of report format $\left(F_{(1,117)}=19.49, p<.001\right.$, $\left.\eta^{2}=.103\right)$ were significant. For procedural justice perceptions, interaction $\left(F_{(1,117)}=53.86, p<.001\right.$, $\left.\eta^{2}=.271\right)$ and main $\left(F_{(1,117)}=25.89, p<.001, \eta^{2}=.132\right)$ effects emerged again. Regarding the report format, we found the anticipated main effect: Participants in the narrative condition perceived more justice than participants in the analytical condition. The interaction effects further suggested that the narrative report format decreased the effect of the personal identity violation on distributive (Figure 52) and procedural (Figure 5-3) justice perceptions.

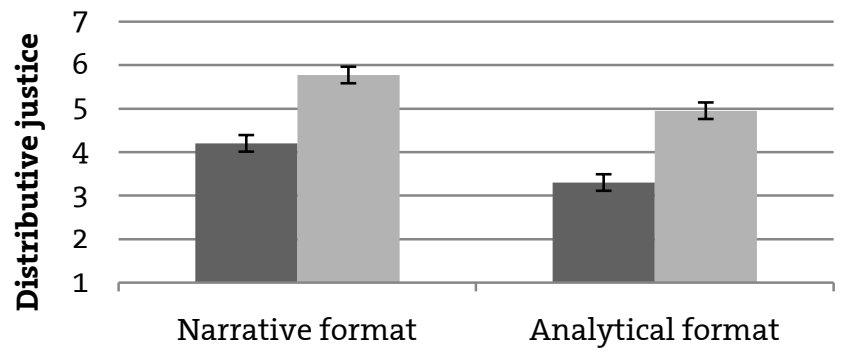

- Personal identity violation

- No personal identity violation

\section{Figure 5-2}

Study 1: Distributive justice for different report format and personal identity violation combinations.

The error bars show the standard error. 


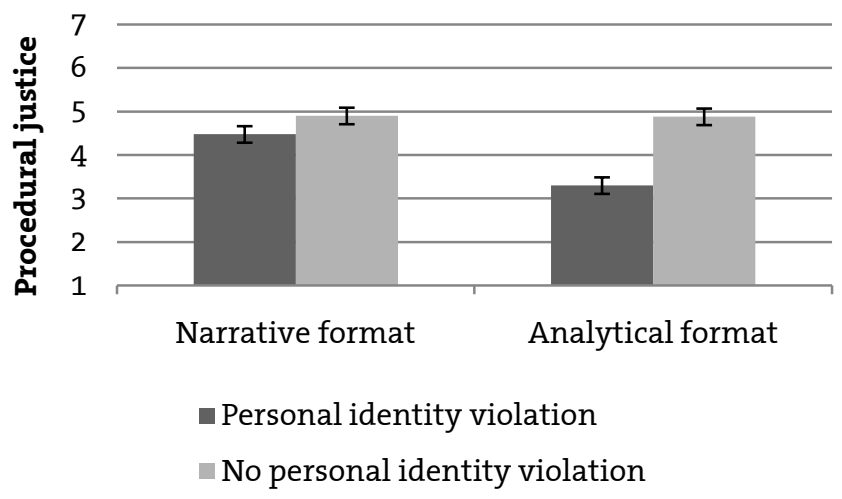

\section{Figure 5-3}

Study 1: Procedural justice for different report format and personal identity violation combinations. The error bars show the standard error.

The simple contrasts showed that in the analytical condition, more distributive (mean difference=1.65, SE=.29, $p<.01)$ and procedural (mean difference $=1.58, \quad \mathrm{SE}=.26, \quad p<.001$ ) justice perceptions appeared when personal identity had not been violated. In contrast, in the narrative condition, the differences between a violated and unviolated personal identity were not significant at the .05 level. This result pattern supports $\mathrm{H}_{1}$.

\section{Discussion}

The overall results of Study 1 show that another user's cyber harassment report, formatted as a narrative, reduces the identity violations associated with privacy regulation decisions. However, social media users who read a cyber harassment report in an analytical format likely feel their personal identity has been violated by restrictive decisions and perceive less distributive and procedural justice. These findings align with previous research on 
analytical report formats (e.g., D. M. Mayer, et al., 2009), as well as with our hypothesis. When consumers read an engrossing narrative account of cyber harassment, they project themselves into the victim's position. This perspective taking leads them to perceive a privacy regulation decision as just. Also, consistent with transportation theory, we find readers of narratives experience more transportation than do people reading an analytical report. When cyber harassment reports fail to evoke transportation, a related restriction affects the reader's identity, and the likelihood that he or she perceives the restriction as just decreases.

Thus, with regard to the impact of cyber harassment reports on the acceptance of privacy regulation decisions, transporting narratives appear crucial as a means to prevent a decrement in justice perceptions. Such reports should put readers in the victim's shoes, which makes them less critical and minimizes the chances that privacy regulation decisions affect the readers' identity. In Study 1, we have not examined the role of consumers' own (social media) identity in these effects though. As Escalas (2007) notes, referencing may drive transportation, in that when a person relates story events to either the self or a public profile, he or she is likely to be transported into the narrative account and may believe that the events are affecting not the victim but the self. Referencing thus could delineate the relationship between narrative transportation in cyber harassment reports and justice perceptions of privacy regulation decisions further. By priming referencing in Study 2, we thus explore the implications of consumers' (private) self or (public) profile in social media. 


\section{Study 2}

In her experiments, Escalas (2007) reports significant positive correlations between the level of transportation and narrative self-referencing. Although her research supports the claim that transportation can be manipulated, she does not disentangle the ways self-referencing might change beliefs. West, Huber, and Min (2004) compare selfand other-referencing and find that self-referencing leads to more story-consistent beliefs, but they do not assess transportation. Empirical research thus far has neither focused on social media nor distinguished (private) selves from (public) profiles.

Conceptual work on self-presentation and selfdisclosure in social media claims though that people often decide to become part of a social network so they can construct an online public profile (Kaplan \& Haenlein, 2010). This profile relates to the self but usually is constructed through communications of thoughts, feelings, likes, and dislikes that are consistent with the social network user's desired public image.

In line with this reasoning, we examine whether an independent mental priming of self-, profile-, or other user-referencing in a cyber harassment report influences perceptions of the distributive and procedural justice of a privacy regulation decision, as mediated by transportation. We predict that profile- or other user-referencing will undermine acceptance of privacy regulation decisions, whereas self-referencing should lead to more transportation and strengthen the effect on justice perceptions (from Study 1). As another test of narrative power, we also check the robustness of the effects across personal and social identity violations 
(e.g., D. M. Mayer, et al., 2009). In summary, we hypothesize that for both personal and social identity violations,

$\mathbf{H}_{2}: \quad$ When social media users refer a cyber harassment report to their private selves, they experience more transportation than when they refer it to their profiles or another user.

$\mathbf{H}_{3}$ : When social media users refer a cyber harassment report to their private selves, they perceive more (a) distributive and (b) procedural justice of a privacy regulation decision.

$\mathbf{H}_{4}: \quad$ Transportation mediates the effect of selfreferencing of the cyber harassment report on (a) distributive and (b) procedural justice perceptions of the privacy regulation decision.

\section{Method}

Similar to the procedure used in Study 1, we asked participants to read online texts. We told them that their university had launched a social networking site and that students had been enrolled. They read wall posts from fellow network members, including accounts of rude and demeaning messages and mean comments. The final post was a message from the network provider about the dramatic increase in offensive content on the social networking site. As our manipulation of the identity violation, the network provider either communicated the decision to remove all users' personal information or general information. The experiment also varied the instructions for approaching the reading task, priming one group to 
relate the posts to the self, a second group to process the posts as if they happened to their public profile, and a third group to imagine the events that took place for another network user. The participants were randomly assigned to the identity violation and referencing conditions. Study 2 thus employs a 2 (identity violation: personal or social) $\times 3$ (referencing: self, profile, or other user) factorial design.

Participants

Of the 233 students of a major British university who participated, $46.8 \%$ were women, and $88.8 \%$ were members of a social networking site. The network members spent on average 22 hours per week using social media. The participants' average age was 25 years.

Manipulation and priming

To test the relationship between the identity violation and the transportation drivers, we created two identity violations: personal and social. For example, the following excerpt represents the personal identity violation: "following the guidelines in our regulations, we decided to remove all your personal information concerning faculties, studies, and student life. From now on, you are no longer allowed to have any information about your faculty, study, or student association on your personal [university name]-Network page." Participants in the social identity violation condition instead read: "following the guidelines in our regulations, we decided to remove all information of all students concerning faculties, studies, and student life. From now on, it is no longer allowed for students to have any information 
about their faculty, study, or student association on their [university name]-Network page." (see Appendices, pp. 219-220).

To distinguish levels of transportation, we used three primes: self-, profile-, and other userreferencing. These conditions follow Green and Brock's (2000) methods to encourage participants to relate the posts to a particular identity. After the study introduction, the participants assigned to the self-referencing condition read, on a computer screen,

The wall posts are about you. You are the student of [university name] the posts are talking about. Your responses will help the network provider to decide on privacy regulations. While reading the wall posts, use your emotion. Think about how you feel. You are on!

The profile-referencing instructions instead encouraged participants to relate the posts to their public profile:

The wall posts are about your network profile. Your profile is the one the posts are talking about. Your responses will help the network provider to decide on privacy regulations. While reading the wall posts, use your emotion. Think about how your image might get hurt in the situation. You are now your profile!

Finally, the other user-referencing group was encouraged to picture the narrative events from the perspective of another network user: 
The wall posts are about Alex. She is the student of [university name] the posts are talking about. Your responses will help the network provider to decide on privacy regulations. While reading the wall posts, use your emotion. Think about how Alex might feel in the situation. You are now Alex!

\section{Measures}

The dependent and control measures are similar to those in Study 1, namely, the extents to which the participants perceive distributive and procedural justice, experience transportation, and tend to become absorbed in life experiences. The need for cognition measure was not relevant, because it pertained to the ability to process information in an analytical format. Neither was the social identification measure, because it could interact with the referencing instructions. In addition to absorption tendency, we assessed the participants' homogeneity using De Cremer's (2002) self-esteem and De Cremer and Tyler's (2005) concern for reputation scales, both which relate to justice perceptions (see Appendices, pp. 221 and 227).

\section{Results}

\section{Manipulation and priming checks}

To check the identity violation manipulation, we used a measure similar to the manipulation in Study 1 . The perceived personal $(\alpha=.89)$ and social identity violation $(\alpha=.86)$ averages differentiated reliably between the manipulation conditions (Wilks' $\lambda=.64, F_{(2,230)}=63.50, p<.001, \eta^{2}=.356$ ).

Participants in both conditions correctly identified either the personal $\left(F_{(1,231)}=95.26, p<.001, \eta^{2}=.292\right.$; mean difference $=1.29, \mathrm{SE}=.09)$ or social $\left(F_{(1,231)}=40.91\right.$, 
$p<.001, \quad \eta^{2}=.150 ; \quad$ mean difference $\left.=.78, \quad \mathrm{SE}=.09\right)$ identity violation.

We also determined participants' understanding of the task while they were reading the story. No participants reported difficulty following the referencing instructions: Selfreferencing participants attempted to picture the narrative events from their own perspective, profile-referencing participants tried to empathize with their public profile, and the other userreferencing participants attempted to picture the perspective of another network user. Thus, the manipulation and priming were successful. In Table 5-3, we list the average scores, reliabilities, and intercorrelations of the dependent and control variables.

\section{Table 5-3}

Study 2: Descriptive statistics.

\begin{tabular}{llllll}
\hline & & $M(\mathrm{SD})$ & $\alpha$ & 1 & 2 \\
\hline 1 & Transportation & $3.63(1.01)$ & .98 & & \\
2 & Distributive justice & $4.38(1.14)$ & .99 & $.68^{* *}$ & \\
& Procedural justice & $3.50(1.31)$ & .97 & $.53^{* *}$ & $.55^{* *}$ \\
& Absorption & $3.98(.95)$ & .91 & & \\
& Self-esteem & $4.01(1.04)$ & .80 & & \\
& Concern for reputation & $3.94(1.02)$ & .89 & & \\
\hline
\end{tabular}

${ }^{* *} p<.01$.

We found no differences in absorption, selfesteem, or concern for reputation across conditions. All groups showed the same tendency to engage in life experiences (Wilks' $\lambda=.94, F_{(15,622)}=.93, p=.536$ ), which indicates that the randomization was successful. 
Hypotheses tests

We present the average scores and standard deviations of transportation, distributive justice, and procedural justice across the personal and social identity violation and self-, profile-, and other userreferencing conditions in Table 5-4.

A $2 \times 3$ MANOVA revealed a main effect of referencing (Wilks' $\lambda=.87, F_{(3,225)}=11.09, \quad p<.001$, $\left.\eta^{2}=.129\right)$ but not of integrity violations at a .05 significance level (Wilks' $\lambda=.95, F_{(6,450)}=1.91, p=.078$ ). The priming conditions created different transportation effects $\left(F_{(2,227)}=11.99, p<.01, \eta^{2}=.050\right)$ and distributive $\left(F_{(2,227)}=33.16, p<.001, \eta^{2}=.127\right)$ and procedural $\left(F_{(2,227)}=10.32, \quad p<.01, \eta^{2}=.044\right)$ justice perceptions.

Simple contrasts showed that self-referencing evoked more transportation than profilereferencing (mean difference $=.58, \mathrm{SE}=.22, p<.01$ ) or other user-referencing (mean difference $=.82, \mathrm{SE}=.21$, $p<.001)$. Self-referencing participants perceived more distributive justice than participants in the profile-referencing (mean difference $=.75, \mathrm{SE}=.24$, $p<.01$ ) or other user-referencing (mean difference $=1.06, \mathrm{SE}=.24, p<.001$ ) conditions. We also found significant differences in procedural justice perceptions between self-referencing and the other conditions (profile: mean difference $=.87, \mathrm{SE}=.28$, $p<.01$; other user: mean difference $=1.26, \mathrm{SE}=.27$, $p<.001$ ). These results strongly support $\mathrm{H}_{2}$ and $\mathrm{H}_{3}$. 


\section{Table 5-4}

Study 2: Transportation, distributive justice, and procedural justice as a function of identity violation and referencing.

\begin{tabular}{|c|c|c|c|c|}
\hline Identity violation & Referencing & $\begin{array}{l}\text { Transportation } \\
M(\mathrm{SD})\end{array}$ & $\begin{array}{l}\text { Distributive justice } \\
M(\mathrm{SD})\end{array}$ & $\begin{array}{l}\text { Procedural justice } \\
M(\mathrm{SD})\end{array}$ \\
\hline \multirow[t]{3}{*}{ Personal } & Self & $4.33(1.05)$ & $4.75(1.02)$ & $4.08(1.24)$ \\
\hline & Profile & $3.60(.90)$ & $4.41(1.05)$ & $3.53(1.09)$ \\
\hline & Other & $3.32(.92)$ & $3.62(1.14)$ & $2.63(1.29)$ \\
\hline \multirow[t]{3}{*}{ Social } & Self & $3.87(.96)$ & $4.90(1.00)$ & $4.03(1.28)$ \\
\hline & Profile & $3.45(.94)$ & $4.62(1.02)$ & $3.79(1.03)$ \\
\hline & Other & $3.28(.90)$ & $3.92(1.09$ & $2.96(1.33)$ \\
\hline
\end{tabular}


For the test of $\mathrm{H}_{4 a}$ and $\mathrm{H}_{4 \mathrm{~b}}$, we conducted mediation analyses and compared the referencing groups across personal and social identity violation conditions. When we included transportation in the referencing-justice perceptions equations as a covariate, the effect on distributive $\left(F_{(2,229)}=.75\right.$, $p=.474)$ and procedural $\left(F_{(2,229)}=1.72, p=.182\right)$ justice perceptions became insignificant. Sobel tests derived from MacKinnon and colleagues (2002) and Zhao and colleagues (2010) offered further support for $\mathrm{H}_{4 \mathrm{a}}(Z=2.01, p<.05)$ and $\mathrm{H}_{4 \mathrm{~b}}(Z=1.96, p<.05)$.

\section{Discussion}

In Study 2, we explore the distinctive influences of self-, profile-, and other user-referencing. With regard to cyber harassment, relating the experience to the self transports consumers more than does profile- or other user-referencing. Transportation has received limited attention in justice research (e.g., Appel, 2008); for example, Green and Brock (2000, Study 3) report a significant effect of transportation on distributive justice perceptions. We add nuance to their findings and disentangle self- and public profile-referencing, in support of assertions that have been left empirically unverified before now (e.g., Sestir \& Green, 2010). In particular, we show in Study 2 that consumers display stronger justice perceptions when they relate a cyber harassment experience to themselves rather than to their profile or another user. Reference to a profile also has a greater impact on transportation and perceptions of distributive and procedural justice than references to other users. Finally, transportation mediates the combined impact of referencing cyber harassment experiences and perceiving privacy regulation decisions as just. To encourage acceptance of privacy regulation 
decisions in social media, self-referencing provides a crucial driver of transportation.

\section{Limitations}

To consider differences among justice perceptions of privacy regulation decisions, we use a narrative account of cyber harassment that creates a narrative profile similar to a story character. However, Brewer and Lichtenstein (1982) highlight two more critical story attributes: causality and chronology. First, a causal sequence of rising and falling events evokes transportation and related evaluations (Adaval \& Wyer Jr., 1998; Green \& Brock, 2000). Conceivably, a greater rise or fall induces more transportation, yet we kept causality constant.

Second, a chronological narrative structure evokes suspense by concealing the outcome, which leads to transportation (Wang \& Calder, 2006). In our studies, the temporal sequence of events was entirely chronological, whereas social media messages in reality often contain links-that is, flashbacks or flash-forwards-that could disrupt transportation by demanding additional processing effort.

Third, this research examines only the effects of self-referencing on self-reported transportation and perceived justice. In a related point, our studies feature only short-term profile-referencing conditions that arise from single priming before a single social media experience, not long-term exposures to profiles constructed over time through repeated interactions. 
Although investigations of identity appear primarily in social psychology, our research suggests that it has clear implications for social media management as well. Social media profiles may provide consumers with a temporary escape from themselves, but this setting does not help relate cyber threats to the self. Moreover, social media may offer insight into the nature of peer-topeer relationships; transportation and selfreferencing may provide the interaction within which this insight emerges. Thus social network providers must remain well aware of the importance of narratives as effective means to end cyber harassment. 



\section{6 \\ GENERAL DISCUSSION}

In the context of integrity violations, the rise of social media grants customers a great opportunity to make their voices heard. This trend suggests that employees' handling of complaints of integrity violations online is a relevant and timely dissertation topic. To explain how employees attend to and process customer-generated narratives, we explore in Chapter 2 what has been called "transportation", the mechanism that underlies narrative processing. We distinguish empathic and imaginative appeals, the main transportation drivers, and support prior assertions that have been left empirically unverified (Green \& Brock, 2002). Driven by this meta-analytic review, we identify in Chapter 3 the need to develop a mental trigger that drives employees to understand and feel for customers who post blogs about integrity violations before they respond to those blog posts, with the goal of encouraging employees to take responsibility for and respond to the violation.

The dramatic proliferation of blogs and their impact on the ways companies compete lead to the pressing need for companies to respond effectively to threats to perceptions of their integrity (see Martin \& Smith, 2008). Preliminary evidence clearly reveals that words speak louder than actions in 
relation to the short-term restoration of integrity because such restorative messages provide a signal of interactional justice (Patterson, Cowley, \& Prasongsukarn, 2006). Explanations for this effect suggest that verbal communication provides meaningful information and a signal of sensitivity to the concerns of consumers. Thus far, though, the identification of which words are more effective and when (i.e., in which conditions) has remained unexplored. In Chapter 4, we find that consumer perceptions of integrity and intentions to switch depend on both response content and format. In line with the findings of Kim, Ferrin and colleagues (2007; 2004), we reveal that denials restore integrity to a significantly higher degree than apologies if framed in an analytical format. To account for this phenomenon, Snyder and Stukas Jr. (1999) argue that apologies tend to be associated with guilt, and people regard guilt admissions as a negative sign after integrity violations. Consumers seem more willing to accept human failures after factual denials because the facts correspond better to a lack of guilt. Despite considerable attention to analytical elaboration in research on persuasive communication (e.g., Petty \& Wegener, 1998), such studies largely ignore narrative responses, which seem to require of readers an emotional effort to process. We argue that the superiority of a narrative apology to a narrative denial may come about because the former facilitates the restoration of beliefs and intentions based on a compelling narrative. An unknown or threatening violator of integrity may become more "human" after a narrative apology. We show both theoretically and empirically that the immediate consequence of such a disarming effect is decreased consumer intention to switch. 
In Chapter 4, we have used transportation theory as well (Green \& Brock, 2002). Empathy, which induces transportation into the feelings of a story character, has a significant, positive impact on perceptions of the integrity of the accused party. Likewise, we find a positive effect for imagining the narrative events. However, this effect is not as strong as that of empathic appeals, meaning that consumers who try to perceive the situation through the alleged evildoer's eyes will consider the accused party more honest than those who develop a helicopter view of the events. In combination with the content of narrative responses, we find that consumers who feel empathy while reading a narrative apology are more likely to restore integrity perceptions than are consumers picturing the events, though the strategies' effects do not differ for consumers confronted with a denial. In that sense, narrative apologies' relatively superior effectiveness appears due to what Escalas and Stern (2003, p. 567) so aptly call the consumer "who vicariously merges with another's feelings." This idea is more than a suggestion, as demonstrated by the comments of a participant in the second experiment of Chapter 4:

To me Adam Klein appears to be a likeable, motivated manager who enjoyed doing his job, but maybe did not fully oversee the seriousness or responsibility linked to his task... His blog entry seems to be honest and truly regretting his behaviour.

The dramatic proliferation of social media and the profound impact of harassment on such networks also demand that social networking site providers manage privacy effectively. From preliminary evidence pertaining to an analytical context, we have learned that restrictive measures 
that seem to target the social part of people's identity appear more just than decisions that seem to aim at people's personal identity (D. M. Mayer, et al., 2009), perhaps because the latter condition causes people to feel singled out unrightfully. However, it remained unclear which decisions would be most effective in response to narrative descriptions of cyber harassment. The results from Chapter 5 show that narrative cyber harassment reports positively affect consumer perceptions of both the distributive and the procedural justice of network provider decisions that may challenge their identity. Specifically, privacy regulation decisions result in a significantly higher degree of perceived justice if presented in response to a cyber harassment report in a narrative instead of an analytical format. Unlike an analytical format, a narrative allows people to take the victim's perspective. Thus, readers become less critical, and the privacy regulation decision affects them less. To account for this phenomenon, Gerrig (1993) argues that narratives evoke transportation, the mechanism underlying narrative processing, and therefore consumers do not associate the effects of the decision with their sense of self. Decisions seem more just when presented in response to cyber harassment reports in a narrative format.

\section{Further research suggestions}

No research has yet assessed the longitudinal aspects of the transportation effect; studies on this topic could reveal fruitful insights into the formation of narrative-based beliefs and their changes over time. They might also clarify the relationship between transportation and transportability. In particular, we anticipate that 
people with different levels of transportability experience different patterns of narrative persuasion, so research should explore these effects using readers from varied samples.

Further research should also assess whether these findings extend to actual levels of transportation and justice. One way to measure "true" transportation would be to use signal detection, in which low-battery signals appear on screen, and researchers record the number of signals that participants detect (Riley, Kaber, \& Draper, 2004).

We can imagine different predictions about the extent to which story-relevant beliefs carry over to real-life beliefs. On the one hand, being transported into a narrative may provide readers with the feeling that their beliefs are based on direct experience, such that they hold their beliefs with great confidence (Fazio \& Zanna, 1981). In this case, transportation would result in a profound change of real-life beliefs. On the other hand, beliefs based on the suspension of disbelief may be deserted soon after the thrill of the story dissipates, such that readers simply restore their prior beliefs. Further research might consider whether involvement goals other than just engrossing entertainment motivate readers (see Rubin, 1994). To understand the effects of different goals, researchers could explore various motivations, including information, surveillance, self-interest assessment, value defence, or value reinforcement (Slater, 1997).

When we examine transportation effects as functions of a rich or poor medium modality in Chapter 2, we find no significant difference. Additional experimental tests could integrate the mental imagery component with hybrid modalities to provide evidence of its predictive validity for 
transportation that is based on mediated stories. Such research likely can explicate how medium modality and mental imagery together influence beliefs and intentions. Related research then might attempt to understand transportation effects in four conditions: high empathy/low mental imagery; low empathy/high mental imagery; both high; and both low (cf., Chapter 4). For marketing scholars, the distinctive effects of appeals to either deep or wide senses suggest an area of interesting research possibilities related to the impact of social media.

Chapter 4 suggests a related moderation, such that the transportation effect pertains to the match between the transportation component and story content. We find that empathy effects tend to be larger than mental imagery effects when readers confront highly emotional content, for which empathy operates as a driver. Further research should also clarify the role of medium attributes in relation to other moderating or mediating component effects. Facebook recently redesigned its news feed feature, so users can view popular content determined by an algorithm based on interest, without reviewing all preceding information to establish a causal chain of events. Therefore, further research should distinguish between the beneficial and harmful uses of causality in social media. In an extreme case of cyber harassment, transportation may be so extensive that consumers ask for restrictions of liberties. Additional research should investigate whether such requests only come from consumers who refer the experience to themselves or from those who possess an elaborate alias as well as whether profiles might become internalized over time. 
Typical blog rolls or walls appear in reverse chronological order, with the newest message on top, which also could limit transportation. These consequences seem somewhat obvious, but social network usage may offer an interesting moderator: If people log on more frequently, they have fewer new messages to read, so they experience less strain due to the lack of chronology. Assessing the true status of this proposition would represent a fruitful research path.

Dual process models of belief and intention changes, such as the Elaboration Likelihood Model (ELM; Petty \& Cacioppo, 1986), have long dominated discussions about the persuasiveness of communications. Slater $(2002 \mathrm{~b})$ and Slater and Rouner $(2002,2003)$ offer some extensions of the ELM (see also Moyer-Gusé \& Nabi, 2010). Extensions that consider the complicated relationships among transportation, story line appeal, and identification with characters, behaviour, and a host of other variables provide a multitude of avenues for inquiry.

Similarly, companies have considerable means to bad-mouth competitors. Attempts to use storytelling systematically to strengthen their own competitive position and call loyal customers to action may inadvertently prompt a remarkable change in competitive markets, even if we ignore the many moral and ethical questions. Thus, research into the consequences and appropriateness of efforts to influence loyal consumers' behaviour in this way is imperative. It may furthermore be interesting to research disloyal consumers within the blogosphere.

Future research should also further investigate the reasons companies either take or pass responsibility for an integrity violation, for as long 
as companies do not attribute blame internally, they will not react, in which case negative word-ofmouth could destroy their reputation. In other words, a more in-depth understanding of the differences in mindsets between companies that search their own conscience and companies that attribute blame to external causes beyond their power offers a fruitful research path.

Additional research should delineate how selfsufficient and exchange-focused mindsets differ conceptually. If tested in combination with a relevant outcome (e.g., money refund), this investigation might provide an interesting empirical approach as well. Focusing on the effects on other employee beliefs and intentions may be an interesting direction for research designed to prime an empathic perspective.

Finally, our study settings may create some confounds. Beyond financial services, healthcare, and retailing, various areas could benefit from research on narratives and transportation effects (e.g., forensic psychology). Ongoing efforts should include other areas to confirm our findings' generalizability.

\section{Managerial implications}

The social media context we investigate is interesting for firms that need to find ways to deal with this relatively new environment and its challenges. Managers must be cognizant of the growing popularity of online stories, particularly as avenues for customer complaints. To restore the integrity perceptions of a complaining customer, the firm cannot simply route complaints to a public relations or web care department, because such 
tactics exclude employees from acknowledging responsibility or responding to the customer. Moreover, a company spokesperson without any involvement in the focal transaction is unlikely to experience transportation. Therefore, instead of a scripted response provided by a customer service representative, implicated employees should transport themselves into, take responsibility for, and respond to posts about them or their actions.

In addition, many firms still rely on financial cues (e.g., exchange and interest rates, financial metrics) that have positive consequences for only some customer segments. A focus on productivity and profitability likely reinforces interest in integrity violations perceived only by customers who are less financially vulnerable. We explored in Chapter 3 whether, irrespective of "doing things right," employees can "do the right thing" (Heide \& Wathne, 2006) for customers who are more vulnerable to the consequences of integrity violations. Against this backdrop, Chapter 3 shows that to evoke employee transportation, firms should create an environment that stimulates their empathic perspective. Firms need frequent, effective triggers that can accelerate an empathic perspective, such as screen savers or Multi-Touch Collaboration Walls that feature empathic priming words (see Figure 6-1). 


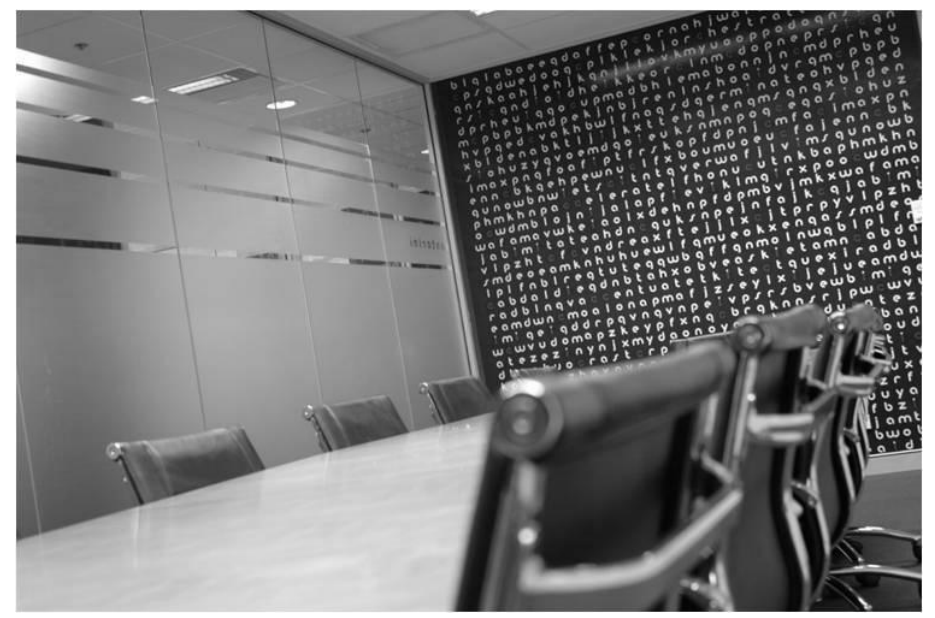

\section{Figure 6-1}

Multi-Touch Collaboration Wall with priming words.

Furthermore, reward and recognition systems, which often drive performance in services industries, should balance economic with empathic criteria to measure success. A perspective taking index might recognize employees for demonstrations of their empathy in the previous quarter. Each action could be judged by a panel of independent experts and employees who demonstrate that they have "saved" a customer from defecting could earn rewards for their responsible, responsive behaviour.

Finally, internal communications, such as newsletters, should prime not only a self-sufficient but also an empathic perspective, such as by highlighting customer service and complaint handling ratings. Firms can expressly use vocabulary that elicits both perspectives; a skilled wordsmith should be hired to develop such materials. Accordingly, it seems important to review hiring practices and recruit people who are 
conversant with social media-such as the Comcast employee who started a Twitter account and thus put "in one deft swoop, a human face on a company that had been disparaged for poor customer service" (Gaines-Ross, 2010, p. 74). More employees might learn to respond in narrative formats and become characters in an unfolding story. Without such efforts, firms will likely produce clinical, standard responses that serve only to alienate customers and thousands of online readers. Employees always have the responsibility to treat customers well; we suggest in Chapter 3 that an empathic perspective in online conversations may enable them even to be transported into blog posts and thus act accordingly. If employees try on the mental shoes of the customer, they may be able to walk much further into their stories.

As aforementioned, companies would be well advised to devote attention to detecting online narratives that indicate negative consumer experiences before those stories cause serious damage (Elsner, et al., 2010; Li, et al., 2004). Firms should provide employees with the necessary resources to monitor blogs that tend to play leading roles in rapid information dispersion across online social networks of consumers. For example, Webclipping (www.webclipping.com), arguably the Internet's most influential social media monitoring service, measures online public opinion. It is managerially relevant to analyze this opinion and determine the level of threat that the blogs represent for the company's reputation.

Furthermore, firms should respond to the blog posts that they deem harmful. Chapter 4's results demonstrate that companies can restore most of their integrity and subsequently retain customers by posting the right combination of response 
content and format in the wake of negative blog stories: either denying responsibility for the matter in an analytical fashion or apologizing in the form of a narrative that triggers consumers' affective reactions. Using pre-developed scripts, employees can follow basic decision rules and arrive at the right response (i.e., analytical denial or narrative apology). Producing restorative narratives is a difficult but well-justified task associated with specific skills and techniques. For example, the skills needed to converse with consumers through narrative communication and empathic sensitivity might be taught through creative narrative writing exercises. This approach also could help acquaint employees with the unique subculture of the blogosphere, with its own specific language (in this case, narrative), values, and customs. As a result, all employees will feel responsible for responding to blog posts and maintaining online peer-to-peer conversations about the company.

As Chapter 4 implies, successful integrity restoration may depend on the people who communicate for the company. Firms therefore should realize that if they respond to an integrity violation with a narrative apology, the consumers' opportunity to feel for the involved party becomes crucial and in effect determines whether they will trust the firm or switch to its competition. From a practical viewpoint, developing and testing concept responses with consumer panels seems logical. Only when consumers can empathize sufficiently with the company does a narrative apology gain a clear advantage over a denial. In summary, we suggest that Chapter 4's evidence represents a clarion call for companies to strengthen their competitive positioning by communicating professionally. When a company ensures that consumers like its narrator, it ensures that in its stories they will trust. 
Chapter 5 also offers several managerial implications for social networking sites that need to create a coherent service strategy that incorporates consumers' online personal branding. Social network providers should work to detect and respond to online narratives of harassment before those stories move in the wrong direction and pose a serious threat of user defection. In particular, they should determine which wall posts and group pages play a leading role in dispersive harassment across social networks of their consumers. Then their privacy regulation decisions should be presented in a matter that makes them stand out from the background chatter, to ensure user satisfaction and loyalty.

Providing these decisions appropriately remains a difficult but well-justified challenge, associated with specific skills and techniques. Chapter 5 demonstrates that social network providers can make decisions to protect network users in response to cyber harassment reports. To draw on their social relationships with consumers, network providers first must be able to recognize cyber harassment; therefore, it may be useful to gain familiarity with forms of narrative communication. We suggest that social networking sites develop explicit policies to recognize narrative cyber harassment reports. In the exceptional case that the harassment is described analytically, the social network sites should present decisions that appeal to consumers' social identities. Scripts could be developed that allow network providers to follow basic rules to arrive at the appropriate presentation.

As Chapter 5's results indicate though, successful online identity management also may depend on the different identities consumers use to engage with social media. If social network 
providers respond to a narrative cyber harassment report, consumers' degree of self-focus is crucial, because it can determine whether they accept the provider's decision as just. From a practical viewpoint, allowing and encouraging consumers to retain their own identities in their profile is a good idea. Only if consumers perceive cyber harassment as something that could happen to them will the network provider's decision to restrict their expression have clear advantages in the form of justice perceptions. In summary, we recommend that social networks review their privacy policies and service strategy, instead of blindly propagating greater freedom of expression in social media. As a shining example, Facebook's privacy policy states clearly that it will delete user accounts deemed fake. 


\section{Conclusion}

The results of this dissertation suggest that the mental representation that social media users have regarding what is right-which might seem relatively stable-can be altered. Schemas about responsibility and justice can be changed, at least temporarily, if people adopt different identities in social media settings. The narrative personae to which people relate allow them to explore integrity and privacy issues and substitute for a perceived lack of trust or justice in analytical reports. Social media also provide outlets for expressing profiles that may not correspond to everyday selves. This dissertation provides but one example of how online expressions have caused the narrative to return to power. 



\section{REFERENCES}

Adaval, R., \& Wyer Jr., R. S. (1998). The role of narratives in consumer information processing. Journal of Consumer Psychology, 7(3), 207.

Aggarwal, P. (2004). The effects of brand relationship norms on consumer attitudes and behavior. Journal of Consumer Research, 31(1), 87-101.

Aggarwal, P., \& Law, S. (2005). Role of relationship norms in processing brand information. Journal of Consumer Research, 32(3), 453-464.

Aggarwal, P., \& Zhang, M. (2006). The moderating effect of relationship norm salience on consumers' loss aversion. Journal of Consumer Research, 33(3), 413-419.

Anschutz, D., Engels, R., Van Leeuwe, J., \& van Strien, T. (2009). Watching your weight? The relations between watching soaps and music television and body dissatisfaction and restrained eating in young girls. Psychology \& Health, 24(9), 1035-1050. 
Appel, M. (2008). Fictional narratives cultivate just-world beliefs. Journal of Communication, 58(1), 62-83.

Appel, M., \& Richter, T. (2007). Persuasive effects of fictional narratives increase over time. Media Psychology, 10(1), 113-134.

Argo, J. J., Zhu, R., \& Dahl, D. W. (2008). Fact or fiction: An investigation of empathy differences in response to emotional melodramatic entertainment. Journal of Consumer Research, 34(5), 614-623.

Baranowski, T., Buday, R., Thompson, D. I., \& Baranowski, J. (2008). Playing for real: Video games and stories for health-related behavior change. American Journal of Preventive Medicine, 34(1), 74-82.

Bargh, J. A. (1997). The automaticity of everyday life. In R. S. Wyer Jr. \& J. A. Bargh (Eds.), The automaticy of everyday life (pp. 1-62). Mahwah, NJ: Lawrence Erlbaum.

Bargh, J. A., \& Barndollar, K. (1996). Automaticity in action: The unconscious as repository of chronic goals and motives. In P. M. Gollwitzer \& J. A. Bargh (Eds.), The psychology of action: Linking cognition and motivation to behavior (pp. 457-481). New York, NY: Guilford.

Bargh, J. A., Gollwitzer, P. M., Lee-Chai, A., Barndollar, K., \& Trötschel, R. (2001). The automated will: Nonconscious activation and pursuit of behavioral goals. Journal of Personality and Social Psychology, 81(6), 1014-1027. 
Baumeister, R. F., \& Leary, M. R. (1995). The need to belong: Desire for interpersonal attachments as a fundamental human motivation. Psychological Bulletin, 117(3), 497-529.

Bernoff, J., \& Schadler, T. (2010). Empowered: Unleash your employees, energize your customers, and transform your business. Boston, MA: Harvard Business School.

Betts, G. H. (1909). The distribution and functions of mental imagery. New York, NY: Columbia University.

Biber, D. (1995). On the role of computational, statistical, and interpretive techniques in multi-dimensional analyses of register variation: A reply to Watson. Text, 15(3), 341-370.

Bilandzic, H., \& Busselle, R. W. (2008). Transportation and transportability in the cultivation of genre-consistent attitudes and estimates. Journal of Communication, 58(3), 508-529.

Borenstein, M., Hedges, L., Higgins, J., \& Rothstein, H. (2005). Comprehensive Meta-analysis (Version 2). Englewood, NJ: Biostat.

Bottom, W. P., Gibson, K., Daniels, S. E., \& Murnighan, J. K. (2002). When talk is not cheap: Substantive penance and expressions of intent in rebuilding cooperation. Organization Science, 13(5), 497-513.

Bowen, J. (2011, January 15). Platform updates: New user object fields, edge.remove event and more. Developer blog 
Retrieved January 18, 2011, from http://developers.facebook.com/blog/post/446

Bozarth, J. D., \& Roberts, R. R. (1972). Signifying significant significance. American Psychologist, 27(8), 774-775.

Bracken, C. C. (2006). Perceived source credibility of local television news: The impact of television form and presence. Journal of Broadcasting \& Electronic Media, 50(4), 723-741.

Braverman, J. (2008). Testimonials versus informational persuasive messages: The moderating effect of delivery mode and personal involvement. Communication Research, 35(5), 666-694.

Brealy, J. (2010). The socialisation of customer experiences UK: 2010. London: RightNow Technologies, Inc.

Brewer, W. F. (1995). Discourse force and empirical studies of literature. In G. Rusch (Ed.), Empirical approaches to literature: Proceedings of the Fourth Biannual Conference of the International Society for the Empirical Study of Literature (pp. 89-95). Siegen: LUMIS-Publications.

Brewer, W. F., \& Lichtenstein, E. H. (1981). Event schemas, story schemas, and story grammars. In J. Long, A. Baddeley \& P. Barnard (Eds.), Attention and Performance IX : 9th international symposium, Cambridge, July 1980, proceedings (pp. 363-379). Hillsdale, NJ: Lawrence Erlbaum. 
Brewer, W. F., \& Lichtenstein, E. H. (1982). Stories are to entertain: A structural-affect theory of stories. Journal of Pragmatics, 6(5-6), 473-486.

Brockner, J., Cremer, D. D., Bos, K. v. d., \& Chen, Y.-R. (2005). The influence of interdependent self-construal on procedural fairness effects. Organizational behavior and human decision processes, 96(2), 155-167.

Brown, J., \& Morgan, J. (2006). Reputation in online auctions: The market for trust. California Management Review, 49(1), 61-81.

Busselle, R. W., \& Bilandzic, H. (2008). Fictionality and perceived realism in experiencing stories: A model of narrative comprehension and engagement. Communication Theory, 18(2), 255-280.

Cacioppo, J. T., Petty, R. E., Feinstein, J. A., \& Jarvis, W. B. G. (1996). Dispositional differences in cognitive motivation: The life and times of individuals varying in need for cognition. Psychological Bulletin, 119(2), 197-253.

Chang, C. (2008). Increasing mental health literacy via narrative advertising. Journal of Health Communication, 13(1), 3755.

Chang, C. (2009). "Being hooked" by editorial content: The implications for processing narrative advertising. Journal of Advertising, 38(1), 21-33. 
Cheung, C. M. K., \& Lee, M. K. O. (2010). A theoretical model of intentional social action in online social networks. Decision Support Systems, 49(1), 24-30.

Cohen-Charash, Y., \& Spector, P. E. (2001). The role of justice in organizations: A meta-analysis. Organizational behavior and human decision processes, 86(2), 278-321.

Colquitt, J. A. (2001). On the dimensionality of organizational justice: A construct validation of a measure. Journal of Applied Psychology, 86(3), 386-400.

Colquitt, J. A., Conlon, D. E., Wesson, M. J., Porter, C. O. L. H., \& Ng, K. Y. (2001). Justice at the millenium: A meta-analytic review of 25 years of organizational justice research. Journal of Applied Psychology, 86(3), 425-445.

Cook, T. D., \& Flay, B. R. (1978). The persistence of experimentally induced attitude change. In L. Berkowitz (Ed.), Advances in Experimental Social Psychology (Vol. 11, pp. 1-57). San Diego, CA: Academic Press.

Csikszentmihalyi, M. (1992). Flow: The psychology of happiness. New York, NY: Harper \& Row.

Dal Cin, S. (2005). The use of stories as persuasive tools. Unpublished doctoral dissertation, University of Waterloo, Waterloo, ON.

Dal Cin, S. (2009). "Transportation" into advocacy messages? Paper presented at the Society for Personality and Social Psychology Conference. 
Dal Cin, S., Gibson, B., Zanna, M. P., Shumate, R., \& Fong, G. T. (2007). Smoking in movies, implicit associations of smoking with the self, and intentions to smoke. Psychological Science, 18(7), 559-563.

Dal Cin, S., Zanna, M. P., \& Fong, G. T. (2004). Narrative persuasion and overcoming resistance. In E. S. Knowles \& J. A. Linn (Eds.), Resistance and persuasion (pp. 175-191). Mahwah, NJ: Lawrence Erlbaum.

Das, S. R., \& Chen, M. Y. (2007). Yahoo! for Amazon: Sentiment extraction from small talk on the web. Management Science, 53(9), 1375-1388.

Davis, M. H. (1983). Measuring individual differences in empathy: Evidence for a multidimensional approach. Journal of Personality and Social Psychology, 44(1), 113126.

De Cremer, D. (2002). The self-relevant implications of distribution rules: When self-esteem and acceptance are influenced by violations of the equity rule. Social Justice Research, 15(4), 327-339.

De Cremer, D., \& Tyler, T. R. (2005). Am I respected or not?: Inclusion and reputation as issues in group membership. Social Justice Research, 18(2), 121-153.

de Graaf, A., Sanders, J., Beentjes, H., \& Hoeken, H. (2007). De rol van identificatie in narratieve overtuiging. Tijdschrift voor Taalbeheersing, 29(3), 237-250. 
de Ruyter, K., \& Brack, A. (1993). European legal developments in product safety and liability: The role of customer complaint management as a defensive marketing tool. International Journal of Research in Marketing, 10(2), 153164.

de Wit, J. B. F., Das, E., \& Vet, R. (2008). What works best: Objective statistics or a personal testimonial? An assessment of the persuasive effects of different types of message evidence on risk perception. Health Psychology, 27(1), 110-115.

De Wulf, K., Odekerken-Schröder, G., \& Iacobucci, D. (2001). Investments in consumer relationships: A cross-country and cross-industry exploration. Journal of Marketing, 65(4), 33-50.

Deighton, J., \& Kornfeld, L. (2009). Interactivity's unanticipated consequences for marketers and marketing. Journal of Interactive Marketing, 23(1), 4-10.

Deighton, J., Romer, D., \& McQueen, J. (1989). Using drama to persuade. Journal of Consumer Research, 16(3), 335-343.

Delgadillo, Y., \& Escalas, J. E. (2004). Narrative word-of-mouth communication: Exploring memory and attitude effects of consumer storytelling. Advances in Consumer Research, 31(1), 186-192.

Denham, B. E. (2004). Toward an explication of media enjoyment: The synergy of social norms, viewing 
situations, and program content. Communication Theory, 14(4), 370-387.

Ditto, P. H., \& Lopez, D. F. (1992). Motivated skepticism: Use of differential decision criteria for preferred and nonpreferred conclusions. Journal of Personality and Social Psychology, 63(4), 568-584.

Dollinger, S. J., Preston, L. A., O'Brien, S. P., \& DiLalla, D. L. (1996). Individuality and relatedness of the self: An autophotographic study. Journal of Personality and Social Psychology, 71(6), 1268-1278.

Doyd, D. M., \& Ellison, N. B. (2007). Social network sites: Definition, history, and scholarship. Journal of ComputerMediated Communication, 13(1), 210-230.

Dunlop, S. M., Wakefield, M., \& Kashima, Y. (2008a). Can you feel it? Negative emotion, risk, and narrative in health communication. Media Psychology, 11(1), 52-75.

Dunlop, S. M., Wakefield, M., \& Kashima, Y. (2008b). The contribution of antismoking advertising to quitting: Intraand interpersonal processes. Journal of Health Communication, 13(3), 250-266.

Edelman, D. C. (2010). Branding in the digital age. Harvard Business Review, 88(12), 62-69.

Eliot, T. S. (1942). Little Gidding. London: Faber and Faber.

Elsner, M. K., Heil, O. P., \& Sinha, A. R. (2010). How social networks influence the popularity of user-generated 
content (Special Report No. 10-206): Marketing Science Institute.

Escalas, J. E. (2004a). Imagine yourself in the product: Mental simulation, narrative transportation, and persuasion. Journal of Advertising, 33(2), 37-48.

Escalas, J. E. (2004b). Narrative processing: Building consumer connections to brands. Journal of Consumer Psychology, 14(1/2), 168-180.

Escalas, J. E. (2007). Self-referencing and persuasion: Narrative transportation versus analytical elaboration. Journal of Consumer Research, 33(4), 421-429.

Escalas, J. E., \& Luce, M. F. (2004). Understanding the effects of process-focused versus outcome-focused thought in response to advertising. Journal of Consumer Research, 31(2), 274-285.

Escalas, J. E., Moore, M. C., \& Britton, J. E. (2004). Fishing for feelings? Hooking viewers helps! Journal of Consumer Psychology, 14(1-2), 105-114.

Escalas, J. E., \& Stern, B. B. (2003). Sympathy and empathy: Emotional responses to advertising dramas. Journal of Consumer Research, 29(4), 566-578.

Farrell, J., \& Rabin, M. (1996). Cheap talk. Journal of Economic Perspectives, 10(3), 103-118.

Fazio, R. H., \& Zanna, M. P. (1981). Direct experience and attitude-behavior consistency. In L. Berkowitz (Ed.), 
Advances in Experimental Social Psychology (Vol. 14, pp. 161-202). San Diego, CA: Academic Press.

Feiereisen, S., Wong, V., \& Broderick, A. J. (2008). Analogies and mental simulations in learning for Really New Products: The role of visual attention. Journal of Product Innovation Management, 25(6), 593-607.

Fenigstein, A., \& Levine, M. P. (1984). Self-attention, concept activation, and the causal self. Journal of Experimental Social Psychology, 20(3), 231-245.

Ferrin, D. L., Kim, P. H., Cooper, C. D., \& Dirks, K. T. (2007). Silence speaks volumes: The effectiveness of reticence in comparison to apology and denial for responding to integrity- and competence-based trust violations. Journal of Applied Psychology, 92(4), 893-908.

Folkes, V. S. (1988). Recent attribution research in consumer behavior: A review and new directions. Journal of Consumer Research, 14(4), 548-565.

Folkes, V. S., \& Kotsos, B. (1986). Buyers' and sellers' explanations for product failure: Who done it? Journal of Marketing, 50(2), 74-80.

Fornell, C., \& Wernerfelt, B. (1987). Defensive marketing strategy by customer complaint management: A theoretical analysis. Journal of Marketing Research, 24(4), 337-346. 
Frank, R. H., Gilovich, T., \& Regan, D. T. (1993). The evolution of one-shot cooperation: An experiment. Ethology \& Sociobiology, 14(4), 247-256.

Gaines-Ross, L. (2010). Reputation warfare. Harvard Business Review, 88(12), 70-76.

Gartner Research (2007, June 26). Hype cycle for media industry publishing, 2007 Retrieved March 18, 2008, from http://www.gartner.com/DisplayDocument?ref=g_search \&id=507926\&subref $=$ simplesearch.

Gergen, K. J., \& Gergen, M. M. (1988). Narrative and the self as relationship. In L. Berkowitz (Ed.), Advances in Experimental Social Psychology (Vol. 21, pp. 17-56). New York, NY: Academic Press.

Gerrig, R. J. (1993). Experiencing narrative worlds: On the psychological activities of reading. New Haven, CO: Yale.

Gilliland, S. W. (1994). Effects of procedural and distributive justice on reactions to a selection system. Journal of Applied Psychology, 79(5), 691-701.

Graesser, A., \& Kreuz, R. (1993). A theory of inference generation during text comprehension. Discourse Processes, 16(1-2), 145-160.

Green, M. C. (2004). Transportation into narrative worlds: The role of prior knowledge and perceived realism. Discourse Processes, 38(2), 247-267. 
Green, M. C. (2006). Narratives and cancer communication. Journal of Communication, 56(s1), S163-S183.

Green, M. C. (2008). Transportation theory. In W. Donsbach (Ed.), International Encyclopedia of Communication (pp. 51705175). Oxford, UK: Wiley-Blackwell.

Green, M. C., \& Brock, T. C. (2000). The role of transportation in the persuasiveness of public narratives. Journal of Personality and Social Psychology, 79(5), 701-721.

Green, M. C., \& Brock, T. C. (2002). In the mind's eye: Transportation-imagery model of narrative persuasion. In M. C. Green, J. J. Strange \& T. C. Brock (Eds.), Narrative impact: Social and cognitive foundations. (pp. 315-341). Mahwah, NJ: Lawrence Erlbaum.

Green, M. C., Brock, T. C., \& Kaufman, G. F. (2004). Understanding media enjoyment: The role of transportation into narrative worlds. Communication Theory, 14(4), 311-327.

Green, M. C., Kass, S., Carrey, J., Herzig, B., Feeney, R., \& Sabini, J. (2008). Transportation across media: Repeated exposure to print and film. Media Psychology, 11(4), 512-539.

Greenwood, D. N. (2007). Are female action heroes risky role models? Character identification, idealization, and viewer aggression. Sex Roles, 57(9-10), 725-732. 
Greenwood, D. N. (2008). Television as escape from self: Psychological predictors of media involvement. Personality and Individual Differences, 44(2), 414-424.

Guber, P. (2007). The four truths of the storyteller. Harvard Business Review, 85(12), 52-59.

Hall, A. (2009). Perceptions of the authenticity of reality programs and their relationships to audience involvement, enjoyment, and perceived learning. Journal of Broadcasting \& Electronic Media, 53(4), 515-531.

Hambleton, R. K. (2001). The next generation of the ITC Test Translation and Adaptation Guidelines. European Journal of Psychological Assessment, 17(3), 164-172.

Hambleton, R. K., Yu, J., \& Slater, S. C. (1999). Fieldtest of the ITC Guidelines for adapting educational and psychological tests. European Journal of Psychological Assessment, 15(3), 270-276.

Harter, J. K., Schmidt, F. L., \& Hayes, T. L. (2002). Business-unitlevel relationship between employee satisfaction, employee engagement, and business outcomes: A metaanalysis. Journal of Applied Psychology, 87(2), 268-279.

Heide, J. B., \& Wathne, K. H. (2006). Friends, businesspeople, and relationship roles: A conceptual framework and a research agenda. Journal of Marketing, 70(3), 90-103.

Hennig-Thurau, T., Malthouse, E. C., Friege, C., Gensler, S., Lobschat, L., Rangaswamy, A., et al. (2010). The impact of 
new media on customer relationships. Journal of Service Research, 13(3), 311-330.

Herring, S. C., Scheidt, L. A., Bonus, S., \& Wright, E. (2004). Bridging the gap: A genre analysis of weblogs. Paper presented at the 37th Hawaii International Conference on System Sciences (HICSS-37), Los Alamitos.

Hewitt, J. P. (2003). Self and society: A symbolic interactionist social psychology (9th ed.). Boston, MA: Allyn and Bacon.

Hirschman, A. O. (1970). Exit, voice, and loyalty: Responses to decline in firms, organizations, and states. Cambridge, MA: Harvard University.

Hoffner, C. A., Levine, K. J., \& Toohey, R. A. (2008). Socialization to work in late adolescence: The role of television and family. Journal of Broadcasting \& Electronic Media, 52(2), 282-302.

Huedo-Medina, T. B., Sánchez-Meca, J., Marín-Martínez, F., \& Botella, J. (2006). Assessing heterogeneity in metaanalysis: $Q$ statistic or I index? Psychological Methods, 11(2), 193-206.

Hullett, C. R., \& Levine, T. R. (2003). The overestimation of effect sizes from $F$ values in meta-analysis: The cause and a solution. Communication Monographs, 70(1), 52-67.

Hunter, J. E., \& Schmidt, F. L. (2004). Methods of meta-analysis: Correcting error and bias in research findings Newbury Park, CA: Sage. 
Itoi, R., Ohbuchi, K.-I., \& Fukuno, M. (1996). A cross-cultural study of preference of accounts: Relationship closeness, harm severity, and motives of account making. Journal of Applied Social Psychology, 26(10), 913-934.

Jaworski, B. J., \& Kohli, A. K. (1993). Market orientation: Antecedents and consequences. Journal of Marketing, 57(3), 53-70.

Johnson, M. D., \& Auh, S. (1998). Customer satisfaction, loyalty, and the trust environment. Advances in Consumer Research, 25(1), 15-20.

Kaplan, A. M., \& Haenlein, M. (2010). Users of the world, unite! The challenges and opportunities of social media. Business Horizons, 53(1), 59-68.

Katz, E. (1957). The two-step flow of communication: An up-todate report on an hypothesis. Public Opinion Quarterly, 21(1), 61-78.

Katz, E., \& Lazarsfeld, P. F. (1955). Personal influence: The part played by people in the flow of mass communications (Vol. 2). Glencoe, Ill: Free Press.

Kim, P. H., Dirks, K. T., Cooper, C. D., \& Ferrin, D. L. (2006). When more blame is better than less: The implications of internal vs. external attributions for the repair of trust after a competence- vs. integrity-based trust violation. Organizational Behavior and Human Decision Processes, 99(1), 49-65. 
Kim, P. H., Ferrin, D. L., Cooper, C. D., \& Dirks, K. T. (2004). Removing the shadow of suspicion: The effects of apology versus denial for repairing competence- versus integritybased trust violations. Journal of Applied Psychology, 89(1), 104-118.

Kleinrock, L. (2004). The Internet rules of engagement: Then and now. Technology in Society, 26(2-3), 193-207.

Konijn, E. A., van der Molen, J. H. W., \& van Nes, S. (2009). Emotions bias perceptions of realism in audiovisual media: Why we may take fiction for real. Discourse Processes, 46(4), 309-340.

Kozinets, R. V., de Valck, K., Wojnicki, A. C., \& Wilner, S. J. S. (2010). Networked narratives: Understanding word-ofmouth marketing in online communities. Journal of Marketing, 74(2), 71-89.

Krasnova, H., Spiekermann, S., Koroleva, K., \& Hildebrand, T. (2010). Online social networks: Why we disclose. Journal of Information Technology, 25(2), 109-125.

LaBrie, J., Collier, A., \& Palmer, A. (2010). The Norton cybercrime report: The human impact. Mountain View, CA: Symantec Corporation.

Lakshmanan, A., \& Krishnan, H. S. (2009). How does imagery in interactive consumption lead to false memory? A reconstructive memory perspective. Journal of Consumer Psychology, 19(3), 451-462. 
LaMarre, H. L., \& Landreville, K. D. (2009). When is fiction as good as fact? Comparing the influence of documentary and historical reenactment films on engagement, affect, issue interest, and learning. Mass Communication and Society, 12(4), 537-555.

Lamm, C., Batson, C. D., \& Decety, J. (2007). The neural substrate of human empathy: Effects of perspective-taking and cognitive appraisal. Journal of Cognitive Neuroscience, 19(1), 42-58.

Landreville, K. D., \& LaMarre, H. L. (2010). Working through political entertainment: How negative emotion and narrative engagement encourage political discussion intent. Communication Quarterly.

Leung, K., Tong, K.-K., \& Lind, E. A. (2007). Realpolitik versus fair process: Moderating effects of group identification on acceptance of political decisions. Journal of Personality and Social Psychology, 92(3), 476-489.

Lewicki, R. J., \& Bunker, B. B. (1996). Developing and maintaining trust in work relationships. In R. M. Kramer \& T. R. Tyler (Eds.), Trust in organization: Frontiers of theory and research (pp. 114-139). Thousand Oaks, CA: Sage.

Li, C., Bernoff, J., \& McHarg, T. (2004). Best practices / Blogging: Bubble or big deal? When and how businesses should use blogs. Cambridge, MA: Forrester Research, Inc.

MacKinnon, D. P., Lockwood, C. M., Hoffman, J. M., West, S. G., \& Sheets, V. (2002). A comparison of methods to test 
mediation and other intervening variable effects. Psychological Methods, 7(1), 83-104.

Mar, R. A., Oatley, K., Hirsh, J., dela Paz, J., \& Peterson, J. B. (2006). Bookworms versus nerds: Exposure to fiction versus nonfiction, divergent associations with social ability, and the simulation of fictional social worlds. Journal of Research in Personality, 40(5), 694-712.

Mar, R. A., Oatley, K., \& Peterson, J. B. (2009). Exploring the link between reading fiction and empathy: Ruling out individual differences and examining outcomes. Communications, 34, 407-428.

Markets in Financial Instruments Directive, European Commission http://ec.europa.eu/internal_market/securities/isd/index _en.htm (2009).

Marsh, E. J., \& Fazio, L. K. (2006). Learning errors from fiction: Difficulties in reducing reliance on fictional stories. Memory \& Cognition, 34(5), 1140-1149.

Martin, K. D., \& Smith, N. C. (2008). Commercializing social interaction: The ethics of stealth marketing. Journal of Public Policy \& Marketing, 27(1), 45-56.

Maslyn, J. M., \& Uhl-Bien, M. (2001). Leader-member exchange and its dimensions: Effects of self-effort and other's effort on relationship quality. Journal of Applied Psychology, 86(4), 697-708. 
Mayer, D. M., Greenbaum, R. L., Kuenzi, M., \& Shteynberg, G. (2009). When do fair procedures not matter? A test of the identity violation effect. Journal of Applied Psychology, 94(1), 142-161.

Mayer, R. C., \& Davis, J. H. (1999). The effect of the performance appraisal system on trust for management: A field quasiexperiment. Journal of Applied Psychology, 84(1), 123-136.

Mazzocco, P. J., Green, M. C., \& Brock, T. C. (2007). The effects of a prior story-bank on the processing of a related narrative. Media Psychology, 10(1), 64-90.

Mazzocco, P. J., Green, M. C., Sasota, J. A., \& Jones, N. W. (2010). This story is not for everyone: Transportability and narrative persuasion. Social Psychological and Personality Science, 1(4), 361-368.

Menon, K., \& Dubé, L. (2007). The effect of emotional provider support on angry versus anxious consumers. International Journal of Research in Marketing, 24(3), 268-275.

Mikulincer, M., Gillath, O., Halevy, V., Avihou, N., Avidan, S., \& Eshkoli, N. (2001). Attachment theory and reactions to others' needs: Evidence that activation of the sense of attachment security promotes empathic responses. Journal of Personality and Social Psychology, 81(6), 12051224.

Moore, V. (2009, August, 4). The fake world of Facebook and Bebo: How suicide and cyber bullying lurk behind the facade of 'harmless fun'. Mail Online Femail. Retrieved 
January,

28 ,

2011,

from

http://www.dailymail.co.uk/femail/article-1204062/The-

fake-world-Facebook-Bebo-How-suicide-cyber-bullying-

$\underline{\text { lurk-facade-harmless-fun.html }}$

Morgan, R. M., \& Hunt, S. D. (1994). The commitment-trust theory of relationship marketing. Journal of Marketing, 58(3), 20.

Morgan, S. E., Harrison, T. R., Long, S. D., Afifi, W. A., Stephenson, M. S., \& Reichert, T. (2005). Family discussions about organ donation: How the media influences opinions about donation decisions. Clinical Transplantation, 19(5), 674682.

Morgan, S. E., Movius, L., \& Cody, M. J. (2009). The power of narratives: The effect of entertainment television organ donation storylines on the attitudes, knowledge, and behaviors of donors and nondonors. Journal of Communication, 59(1), 135-U124.

Moyer-Gusé, E. (2008). Toward a theory of entertainment persuasion: Explaining the persuasive effects of entertainment-education messages. Communication Theory, 18(3), 407-425.

Moyer-Gusé, E., \& Nabi, R. L. (2010). Explaining the effects of narrative in an entertainment television program: Overcoming resistance to persuasion. Human Communication Research, 36(1), 26-52. 
Mudambi, S. M., \& Schuff, D. (2010). What makes a helpful online review? A study of customer reviews on Amazon.com. MIS Quarterly, 34(1), 185-200.

Mullen, M. R. (1995). Diagnosing measurement equivalence in cross-national research. Journal of International Business Studies, 26(3), 573-596.

Muniz Jr., A. M., \& O'Guinn, T. C. (2001). Brand community. Journal of Consumer Research, 27(4), 412-432.

Nabi, R. L., \& Krcmar, M. (2004). Conceptualizing media enjoyment as attitude: Implications for mass media effects research. Communication Theory, 14(4), 288-310.

Nabi, R. L., Stitt, C. R., Halford, J., \& Finnerty, K. L. (2006). Emotional and cognitive predictors of the enjoyment of reality-based and fictional television programming: An elaboration of the uses and gratifications perspective. Media Psychology, 8(4), 421-447.

Nell, V. (1988). Lost in a book: The psychology of reading for pleasure. New Haven, CT: Yale University.

Niederdeppe, J., Bu, Q. L., Borah, P., Kindig, D. A., \& Robert, S. A. (2008). Message design strategies to raise public awareness of social determinants of health and population health disparities. Milbank Quarterly, 86(3), 481-513.

Oatley, K. (2002). Emotions and the story worlds of fiction. In T. C. Brock, M. C. Green \& J. J. Strange (Eds.), Narrative impact: 
Social and cognitive foundations. (pp. 39-69). Mahwah, NJ: Lawrence Erlbaum.

Ohbuchi, K.-i., Kameda, M., \& Agarie, N. (1989). Apology as aggression control: Its role in mediating appraisal of and response to harm. Journal of Personality and Social Psychology, 56(2), 219-227.

Oliver, R. L., \& DeSarbo, W. S. (1988). Response determinants in satisfaction judgments. Journal of Consumer Research, 14(4), 495-507.

Patterson, P. G., Cowley, E., \& Prasongsukarn, K. (2006). Service failure recovery: The moderating impact of individuallevel cultural value orientation on perceptions of justice. International Journal of Research in Marketing, 23(3), 263277.

Payne, S. C., \& Webber, S. S. (2006). Effects of service provider attitudes and employment status on citizenship behaviors and customers' attitudes and loyalty behavior. Journal of Applied Psychology, 91(2), 365-378.

Pennebaker, J. W., Mehl, M. R., \& Niederhoffer, K. G. (2003). Psychological aspects of natural language use: Our words, our selves. Annual Review of Psychology, 54(1), 547.

Periatt, J. A., LeMay, S. A., \& Chakrabarty, S. (2004). The selling orientation-customer orientation (SOCO) scale: Crossvalidation of the revised version. Journal of Personal Selling \& Sales Management, 24(1), 49-54. 
Petrican, R., Moscovitch, M., \& Schimmack, U. (2008). Cognitive resources, valence, and memory retrieval of emotional events in older adults. Psychology and Aging, 23(3), 585594.

Petrova, P. K., \& Cialdini, R. B. (2005). Fluency of consumption imagery and the backfire effects of imagery appeals. Journal of Consumer Research, 32(3), 442-452.

Petty, R. E., \& Cacioppo, J. T. (1986). Communication and persuasion: Central and peripheral routes to attitude change. New York, NY: Springer.

Petty, R. E., Cacioppo, J. T., \& Schumann, D. (1983). Central and peripheral routes to advertising effectiveness: The moderating role of involvement. Journal of Consumer Research, 10(2), 135-146.

Petty, R. E., \& Wegener, D. T. (1998). Attitude change: Multiple roles for persuasion variables. In D. T. Gilbert, S. T. Fiske \& G. Lindzey (Eds.), The handbook of social psychology (pp. 323-390). Boston, MA: McGraw-Hill.

Pew Internet \& American Life Project (2006). Usage over time Retrieved March 18, 2008, from http://www.pewinternet.org/Trend-Data/Usage-OverTime.aspx

Pham, M. T., Goukens, C., Lehmann, D. R., \& Stuart, J. A. (2010). Shaping customer satisfaction through self-awareness cues. Journal of Marketing Research, 47(5), 920-932. 
Pitcher, G. (2007, July 26). One in 10 workers experiences cyberbullying in the workplace. Personneltoday Latest news. Retrieved February 24, 2011, from http://www.personneltoday.com/articles/2007/07/26/417 07/one+in+10+workers+experiences+cyberbullying+in+the+workplace.html

Plender, J. (2009, March, 3). Error-laden machine. Financial Times, p. 10. Retrieved September 23, 2009, from London Archive.

Polichak, J. W., \& Gerrig, R. J. (2002). "Get up and win!" Participatory responses to narrative. In T. C. Brock, M. C. Green \& J. J. Strange (Eds.), Narrative impact: Social and cognitive foundations. Mahwah, NJ: Lawrence Erlbaum.

Purdy, D. (2011, January 18). Improvements to permissions for address and mobile number. Developer blog Retrieved January 18, 2011, from http://developers.facebook.com/blog/post/447

Raney, A. A. (2004). Expanding disposition theory: Reconsidering character liking, moral evaluations, and enjoyment Communication Theory, 14(4), 348-369.

Raphael, J. R. (2009, February 17). Facebook privacy change sparks federal complaint. PCWorld Today. Retrieved January 14, 2011, from http://www.pcworld.com/article/159703/facebook_privac y_change_sparks_federal_complaint.html?tk=rel_news 
Rawls, J. B. (1971). A theory of justice (1st ed.). Cambridge, MA: Belknap.

Rawls, J. B. (1999). A theory of justice (2nd ed.). Cambridge, MA: Belknap.

Reid, A., \& Deaux, K. (1996). Relationship between social and personal identities: Segregation or integration. Journal of Personality and Social Psychology, 71(6), 1084-1091.

Reynolds, S. J. (2008). Moral attentiveness: Who pays attention to the moral aspects of life? Journal of Applied Psychology, 93(5), 1027-1041.

Riley, J. M., Kaber, D. B., \& Draper, J. V. (2004). Situation awareness and attention allocation measures for quantifying telepresence experiences in teleoperation. Human Factors and Ergonomics in Manufacturing, 14(1), 51-67.

Rosenthal, R. (1991). Meta-analytic procedures for social research (Revised ed.). Newbury Park, CA: Sage.

Rubin, A. M. (1994). Media uses and effects: A uses and gratifications perspective. In J. Bryant \& D. Zillmann (Eds.), Media effects : advances in theory and research (1st ed., pp. 417-436). Mahwah, NJ: Lawrence Erlbaum.

SAS (2009, June 22-23). Customer Experience Maturity Monitor Research. Paper presented at the Forrester's Customer Experience Forum, New York, NY. 
Saxe, R., \& Weitz, B. A. (1982). The SOCO scale: A measure of the customer orientation of salespeople. Journal of Marketing Research, 19(3), 343-351.

Schank, R. C. (1979). Interestingness: Controlling inferences. Artificial Intelligence, 12(3), 273-297.

Schau, H. J., \& Gilly, M. C. (2003). We are what we post? Selfpresentation in personal web space. Journal of Consumer Research, 30(3), 385-404.

Schellens, P. J., \& de Jong, M. (2004). Argumentation schemes in persuasive brochures. Argumentation, 18(3), 295-323.

Schlosser, A. E. (2003). Experiencing products in the virtual world: The role of goal and imagery in influencing attitudes versus purchase intentions. Journal of Consumer Research, 30(2), 184-198.

Schwarz, N. (1990). Feelings as information: Informational and motivational functions of affective states. In E. T. Higgins \& R. M. Sorrentino (Eds.), Handbook of motivation and cognition (Vol. 2, pp. 527-561). New York, NY: Guilford.

Schwarz, N., \& Wyer Jr., R. S. (1985). Effects of rank ordering stimuli on magnitude ratings of these and other stimuli. Journal of Experimental Social Psychology, 21(1), 30-46.

Scott, L. M. (1993). Spectacular vernacular: Literacy and commercial culture in the postmodern age. International Journal of Research in Marketing, 10(3), 251-275. 
Searls, D., \& Weinberger, D. (2009). Markets are conversations. In R. Levine, C. Locke, D. Searls \& D. Weinberger (Eds.), The cluetrain manifesto: 10th anniversary edition (pp. 147186). New York, NY: Basic Books.

Sestir, M., \& Green, M. C. (2010). You are who you watch: Identification and transportation effects on temporary self-concept. Social Influence, 5(4), 272-288.

Sheehan, P. W. (1967). A shortened form of Betts' questionnaire upon mental imagery. Journal of Clinical Psychology, 23(3), 386-389.

Shrum, L. J. (2009). Television viewing, materialism, and life satisfaction: Mediating and moderating processes in the cultivation effect.Unpublished manuscript.

Shrum, L. J., Burroughs, J. E., \& Rindfleisch, A. (2005). Television's cultivation of material values. Journal of Consumer Research, 32(3), 473-479.

Simmons, R., Birchall, J., \& Prout, A. (2007). Hearing voices: User involvement in public services. Consumer Policy Review, 17(5), 234-240.

Singhal, A., \& Rogers, E. M. (2002). A theoretical agenda for entertainment-education. Communication Theory, 12(2), 117-135.

Skitka, L. J. (2003). Of different minds: An accessible identity model of justice reasoning. Personality \& Social Psychology Review, 7(4), 286-297. 
Slater, M. D. (1997). Persuasion processes across receiver goals and message genres. Communication Theory, 7(2), 125148.

Slater, M. D. (2002a). Entertainment education and the persuasive impact of narratives. In T. C. Brock, M. C. Green \& J. J. Strange (Eds.), Narrative impact: Social and cognitive foundations (pp. 157-181). Mahwah, NJ: Lawrence Erlbaum.

Slater, M. D. (2002b). Involvement as goal-directed strategic processing: Extending the Elaboration Likelihood Model. In J. P. Dillard \& M. Pfau (Eds.), The persuasion handbook: Developments in theory and practice (pp. 175-194). Thousand Oaks, CA: Sage.

Slater, M. D., Buller, D. B., Waters, E., Archibeque, M., \& LeBlanc, M. (2003). A test of conversational and testimonial messages versus didactic presentations of nutrition information. Journal of Nutrition Education and Behavior, 35(5), 255-259.

Slater, M. D., \& Rouner, D. (1996). Value-affirmative and valueprotective processing of alcohol education messages that include statistical evidence or anecdotes. Communication Research, 23(2), 210-235.

Slater, M. D., \& Rouner, D. (2002). Entertainment-education and elaboration likelihood: Understanding the processing of narrative persuasion. Communication Theory, 12(2), 117244. 
Slater, M. D., \& Rouner, D. (2003). The processing of narrative education containing implicit persuasive content about alcohol use: A test of the Extended Elaboration Likelihood Model.Unpublished manuscript.

Slater, M. D., Rouner, D., \& Long, M. (2006). Television dramas and support for controversial public policies: Effects and mechanisms. Journal of Communication, 56(2), 235-252.

Sledgianowski, D., \& Kulviwat, S. (2009). Using social network sites: The effects of playfulness, critical mass and trust in a hedonic context. Journal of Computer Information Systems, 49(4), 74-83.

Small, D. A., Loewenstein, G., \& Slovic, P. (2007). Sympathy and callousness: The impact of deliberative thought on donations to identifiable and statistical victims. Organizational Behavior and Human Decision Processes, 102(2), 143-153.

Smeesters, D., Wheeler, S. C., \& Kay, A. C. (2009). The role of interpersonal perceptions in the prime-to-behavior pathway. Journal of Personality \& Social Psychology, 96(2), 395-414.

Smith, E. R. (1990). Content and process specificity in the effects of prior experiences. In T. K. Skrull \& R. S. Wyer Jr. (Eds.), Advances in Social Cognition (Vol. 3, pp. 1-60). Hillsdale, NJ: Lawrence Erlbaum.

Snyder, M., \& Stukas Jr., A. A. (1999). Interpersonal processes: The interplay of cognitive, motivational, and behavioral 
activities in social interaction. Annual Review of Psychology, 50(1), 273.

Steele, C. M. (1988). The psychology of self-affirmation: Sustaining the integrity of the self. In L. Berkowitz (Ed.), Advances in Experimental Social Psychology (Vol. 21, pp. 261-302). New York, NY: Academic Press.

Steen, G. (1998). Narrative discourse.Unpublished manuscript, Tilburg.

Steuer, J.-S. (1992). Defining virtual reality: Dimensions determining telepresence. Journal of Communication, 42(4), 73-93.

Stowe, C. E., \& Stowe, L. B. (1911). Harriet Beecher Stowe: The story of her life. London: James Nisbet.

Strange, J. J. (2002). How fictional tales wag real-world beliefs: Models and mechanisms of narrative influence. In T. C. Brock, M. C. Green \& J. J. Strange (Eds.), Narrative impact: Social and cognitive foundations. (pp. 263-286). Mahwah, NJ: Lawrence Erlbaum.

Tajfel, H., \& Turner, J. (2004). An integrative theory of intergroup conflict. In M. J. Hatch \& M. Schultz (Eds.), Organizational identity: A reader (pp. 56-65). Oxford: Oxford University.

Tal-Or, N., Boninger, D. S., Poran, A., \& Gleicher, F. (2004). Counterfactual thinking as a mechanism in narrative 
persuasion. Human Communication Research, 30(3), 301328.

Tang, T., Sutarso, T., Davis, G., Dolinski, D., Ibrahim, A., \& Wagner, S. (2008). To help or not to help? The good Samaritan effect and the love of money on helping behavior. Journal of Business Ethics, 82(4), 865-887.

Tanner, L. (2004, November 11). Doctors urged to say 'I'm sorry'. Apologies could help avoid malpractice suits. CBS News Retrieved July 14, 2008, from http://www.cbsnews.com/stories/2004/11/11/health/mai $\underline{\text { n655219.shtml. }}$

Tellegen, A., \& Atkinson, G. (1974). Openness to absorbing and self-altering experiences ('absorption'), a trait related to hypnotic susceptibility. Journal of Abnormal Psychology, 83(3), 268-277.

Terry, D. J., \& Hogg, M. A. (1996). Group norms and the attitudebehavior relationship: A role for group identification. Personality and Social Psychology Bulletin, 22(8), 776-793.

The Economist (2006, April 22). It's the links, stupid, 379, 5-8.

The Economist (2010, May 22). Dicing with data, 395, 16-16.

Trabasso, T., \& Sperry, L. L. (1985). Causal relatedness and importance of story events. Journal of Memory and Language, 24(5), 595-611.

Trusov, M., Bucklin, R. E., \& Pauwels, K. (2009). Effects of wordof-mouth versus traditional marketing: Findings from an 
Internet social networking site. Journal of Marketing, 73(5), 90-102.

Urban, G. L., Amyx, C., \& Lorenzon, A. (2009). Online trust: State of the art, new frontiers, and research potential. Journal of Interactive Marketing, 23(2), 179-190.

van den Hende, E. A., Schoormans, J. P. L., Morel, K. P. N., Lashina, T., van Loenen, E., \& de Boevere, E. I. (2007). Using early concept narratives to collect valid customer input about breakthrough technologies: The effect of application visualization on transportation. Technological Forecasting and Social Change, 74(9), 1773-1787.

van Laer, T. (2005). How the mechanisms underlying analytical and narrative persuasion differ. Unpublished master's thesis, University of Nijmegen, Nijmegen.

van Laer, T., \& de Ruyter, K. (2010). In stories we trust: How narrative apologies provide cover for competitive vulnerability after integrity-violating blog posts. International Journal of Research in Marketing, 27(2), 164174.

Vandenberghe, C., Bentein, K., Michon, R., Chebat, J.-C., Tremblay, M., \& Fils, J.-F. (2007). An examination of the role of perceived support and employee commitment in employee-customer encounters. Journal of Applied Psychology, 92(4), 1177-1187. 
Vaughn, L. A. (2010). Regulatory fit and persuasion: Roles of elaboration and transportation likelihood. In J. C. Magyar \& A. Magyar (Eds.), Psychology of persuasion: Nova Science.

Vaughn, L. A., Hesse, S. J., Petkova, Z., \& Trudeau, L. (2009). "This story is right on": The impact of regulatory fit on narrative engagement and persuasion. European Journal of Social Psychology, 39(3), 447-456.

Vaughn, L. A., Petkova, Z., Hesse, S. J., Trudeau, L., \& McCaffrey, N. E. (2006). Transportation into narrative worlds: The role of processing fluency.Unpublished manuscript, Ithaca, NY.

Vaughn, L. A., Petkova, Z., Hesse, S. J., Trudeau, L., \& Ozses, M. (2007). When it feels easy to enter a story: Processing fluency and mental transportation via narratives.Unpublished manuscript, Ithaca, NY.

Vipond, D., \& Hunt, R. A. (1984). Point-driven understanding: Pragmatic and cognitive dimensions of literary reading. Poetics, 13(3), 261-277.

Vohs, K. D., Mead, N. L., \& Goode, M. R. (2006). The psychological consequences of money. Science, 314(5802), 1154-1156.

Vorderer, P., Klimmt, C., \& Ritterfeld, U. (2004). Enjoyment: At the heart of media entertainment. Communication Theory, 14(4), 388-408.

Walters, C. (2009, February 15). Facebook's new terms of service: "We can do anything we want with your content. Forever.". The Consumerist Your rights. Retrieved January 
http://consumerist.com/2009/02/facebooks-new-termsof-service-we-can-do-anything-we-want-with-yourcontent-forever.html

Wang, J., \& Calder, B. J. (2006). Media transportation and advertising. Journal of Consumer Research, 33(2), 151-162.

Wang, J., \& Calder, B. J. (2009). Media engagement and advertising: Transportation, matching, transference and intrusion. Journal of Consumer Psychology, 19(3), 546-555.

Ward, J. C., \& Ostrom, A. L. (2006). Complaining to the masses: The role of protest framing in customer-created complaint web sites. Journal of Consumer Research, 33(2), 220-230.

West, P. M., Huber, J., \& Min, K. S. (2004). Altering experienced utility: The impact of story writing and self-referencing on preferences. Journal of Consumer Research, 31, 623-630.

Wiertz, C., \& de Ruyter, K. (2007). Beyond the call of duty: Why customers contribute to firm-hosted commercial online communities. Organization Studies, 28(3), 347-376.

Winterbottom, A., Bekker, H. L., Conner, M., \& Mooney, A. (2008). Does narrative information bias individual's decision making? A systematic review. Social Science \& Medicine, 67(12), 2079-2088.

Woodside, A. G., Sood, S., \& Miller, K. E. (2008). When consumers and brands talk: Storytelling theory and research in 
psychology and marketing. Psychology \& Marketing, 25(2), 97-145.

Xie, Y., \& Peng, S. (2009). How to repair customer trust after negative publicity: The roles of competence, integrity, benevolence, and forgiveness. Psychology and Marketing, 26(7), 572-589.

Zhao, X., Lynch Jr., J. G., \& Chen, O. (2010). Reconsidering Baron and Kenny: Myths and truths about mediation analysis. Journal of Consumer Research, 37(2), 197-206.

Zhou, X., Vohs, K. D., \& Baumeister, R. F. (2009). The symbolic power of money: Reminders of money alter social distress and physical pain. Psychological Science, 20(6), 700-706.

Zillmann, D., \& Bryant, J. (1994). Entertainment as media effect. In J. Bryant \& D. Zillmann (Eds.), Media effects: Advances in theory and research (pp. 437-462). Mahwah, NJ: Lawrence Erlbaum. 


\section{INDEX}

acknowledgment of

responsibility, 61-63, 66-67

affective response, 17, 32, 40

apology, 96, 101-104, 108-120, 124

behavioural intention, 19 , 33,40

belief, 18, 33, 40

cognitive response, 17-18, 32,40

communication, 12, 25, 29 , 45-52, 99, 105, 112

cyber harassment, 127-144, 151-153

denial, 62, 95-119

distributive justice, see justice empathy, 14, 20, 27-29, 37, 43-45, 49-50, 57-58, 64-71, 74-79, 91-92, 103, $112-125$

harassment, see cyber harassment

identity violation, 127-133, 136-153

imagery, see mental imagery

integrity

perception, 99, 104, 106-110, 113-120, 123-125

violation, 59-63, 99-102

intention

behavioural, 19, 33, 40 to respond, 62-63, 66-67 to switch, 103-111 
justice, 127-144, 147-151

mental imagery, 14, 20, 27-29, 37, 43-45, 49-50

meta-theory, 11-13, 19-21, 49-51

narrative

content, 3-4, 120

format, 4-5, 25, 35-38, 74-75, 97-98, 103-105, 112, 129-130, 133-134

ownership, 59-63, 66-68, 93

perceived integrity, 99, 104, 106-110, 113-120, 123-125

perspective, 57, 64-66, 80,91

persuasion, see belief

privacy, 130, 134-135, 141-147, 151-152

procedural justice, see justice

profile-referencing, see referencing

referencing, 143-153 response

affective, 17, 32, 40

cognitive, 17-18, 32, 40

responsibility

acknowledgment of, 61-63, 66-67

self-referencing, see referencing

self-sufficiency, $64,68,72$, 75-79

social media, 55-56, 63, 66, 92, 127-136, 141-145, 152-153

transportation, 11, 14, 55-57, 63-64, 97-98, $130,133-134,137-138$

trust, see integrity

violation identity, 127-133, 136-153

integrity, 59-63, 99-102

willingness to respond, 62-63, 66-67 to switch, 103-111 


\section{APPENDICES $^{8}$}

\section{A WALK IN CUSTOMERS' SHOES}

\section{Pre-test and study}

\section{Integrity violation}

[...] Each year, I saved for retirement as much as I could. So I was beside myself with joy when I met this financial magician, who just conjured up an investment portfolio out of his hat with a nice yearly return. Then the poor fellow did a disappearing act, and the high returns he promised turned out to be an illusion too. My investment portfolio has evaporated. The government pension will not be enough to live off. Will I nevertheless have to rely on it? [...] They say that timing is everything-and how. My numbers lined up half a year ago, and I decided to wait to convert everything to the safety of bonds. A few months ago, my net worth was half of what it had been. Time was almost up. When I received the bad news, at first I really thought something like, I won't be able to manage all this. But later I thought something more like: Not to worry, I have ridden out dips before. But at that moment, the way things were financially, I didn't really have a choice [...] But

\footnotetext{
${ }^{8}$ All complete scenarios are available on request from returnofthenarrative@gmail.com
} 
not this time. The ride got too rough. The bank was not very considerate ... a bank that itself advised a heavy investment portfolio. If I worked at your bank, I would never advise anyone to invest such a large sum of money [...] Only time will tell, but I don't think I have enough time to recover. Scary isn't it. I have nothing now, and will have even less tomorrow. A person has to have faith in the future, but as my investment portfolio went, so did my future. I have no more faith. I am doomed.

\section{IN STORIES WE TRUST}

\section{Study 1}

Initial blog post

[...] I am pleased with the obstetrician that I chose. In fact, Adam and I are both VERY pleased. [...] I'm so relieved. I was worried I might have to see several doctors before Adam and I found one that we both liked and who was willing to at least let me try for a natural birth. But I have a tremendous peace about this. [...] He's awesome. I'm feeling good about it. We felt that we are really on the same level here $[\ldots]$.

\section{Integrity violation}

[...] we went to the hospital, but when we were there, we had Dr. Jacobs - the obstetrician - tell us that he was not going to do a natural birth! I thought I wasn't hearing right! This was not what we discussed during the intake. Before we practically knew it, Dr. Jacobs did an ultrasound of Sebastian, measured some things and said that it was a big baby, so we couldn't go natural, since it 
would be inefficient, because the process was going to take up too much time. [...] I had already had one drug in IV, and now Dr. Jacobs was going to give me the epidural, which I had not agreed to take, just to make me shut up! [...] Anyways, the final crew came in, and finally there was Dr. Jacobs again, who had obviously been at home being on call (judging from the clothes he was wearing) and he decided that it was time to get the baby. [...] They let me push for at least a whole 3 minutes when without any occasion, they got the vacuum and the scissors because the baby needed to come out! Now, was there any medical reason for it? Was it obvious that the baby was in distress? I never heard anything about that. Instead, Dr. Jacobs made sure we knew he wanted to go back to the dinner table ASAP [...] He was like 3250 grams, and that's like an average baby. One of the nurses actually told us that a baby this size could have easily been delivered in the slower natural way. By that time, Dr. Jacobs was out the door already! [...] I don't really think that Dr. Jacobs is fit to deliver babies! He clearly broke his promise and commitment he made to our way [...]

\section{Analytical apology}

[...] Parents need to be able to explicitly choose what they share, and obstetricians should not have the right to overrule a birth plan. The World Health Organization estimates that

- Worldwide yearly 110,000 birth accidents happen;

- $85 \%$ of these are related to obstetricians imposing their will upon parents; 
- Observing birth plans to the letter in all cases has the potential to decrease the number of accidents by $8.6 \%$.

These are the statistics that my actions should have been based upon. And who am I not to respect these numbers? I agree with Mrs. Klein's account of her delivery on all points. It is an accurate account of what happened. The birth plan was disregarded. In fact, I only followed up on a small $25 \%$ of the ideas listed in the plan, and I apologize for ignoring the other $75 \%$ [...].

Analytical denial

[...] Parents need to be able to explicitly choose what they share, but obstetricians should have the right to overrule a birth plan. The World Health Organization estimates that

- Worldwide yearly 110,000 birth accidents happen;

- $85 \%$ of these are related to parents imposing their will upon obstetricians;

- Observing birth plans to the letter in all cases has the potential to increase the number of accidents by $8.6 \%$.

These are the statistics that my actions were based upon. And who am I not to respect these numbers? I disagree with Mrs. Klein's account of her delivery on a number of points. It is not an accurate account of what happened. I deny disregarding the birth plan. In fact, I followed up on a substantial $25 \%$ of the ideas listed in the plan, and I have no reason to apologize for ignoring the other $75 \%$ [...]. 
Narrative apology

[...] The actual labour was not a rewarding experience. I was exhausted, because I had rushed in from home being on call. When I came into the delivery room, I believed the baby was in distresssince it had taken me so long to get to the hospitalso then, after 15 minutes of needless pushing and screaming, I used the vacuum and scissors. There was obviously no clear medical reason for doing that. I guess I then intuitively took action. Within 5 minutes, I got the baby out; his face swollen and red, as were both of his arms. In the midst of the chaos, I did not have total control over the procedure. After Sebastian was born, I felt empty and weak. I handed him off and he was whisked away to the incubators.

So yes, I disregarded the philosophy behind the birth plan completely. When I met Laura and Adam, I told them that I would help them bring Sebastian into this world. Last week Laura delivered her baby Sebastian without any of her wishes met, and today I still feel bad about it. I completely agree with Laura's story of her delivery. I've made a lot of mistakes delivering this child. I simply did a bad job with this birth, and I apologize for that [...].

Narrative denial

[...] The actual labour was a rewarding experience. I was still in perfect shape, though I had rushed in from home being on call. When I came into the delivery room, I believed the baby was in distress-since it had taken Laura so long to be induced-so then, after 15 minutes of needless pushing and screaming, I used the vacuum and scissors to get the baby out. There was obviously a clear medical reason for doing that. I guess I then automatically took action. Within 5 minutes, I got 
the baby out; his face and arms only a little flushed. In the midst of the chaos, I had total control over the procedure. After Sebastian was born, I felt empowered and strong. I handed him off and he was whisked away to the incubators.

So yes, I respected the philosophy behind the birth plan completely. When I met Laura and Adam, I told them that I would help them bring Sebastian into this world. Last week Laura delivered Sebastian without any major complications, and today I still feel proud of it. I do not completely agree with Laura's story of her delivery. I deny making any mistakes delivering this child. I did a good job with this birth, and I have no reason to apologize for that [...].

\section{Study 2}

Narrative apology

[...] From those to whom much is given, much is expected. I have been given much-the love of my family, the faith and trust of the people of this country, and the chance to lead this company. I am deeply sorry that I did not live up to what was expected of me. To every train passenger-and to all those who believed in what I tried to stand for-the accusation is true. I am guilty of transferring funds to my private account, and I sincerely apologize.

When I first entered the railway's office complex, I was the epitome of the classic manager: young, brash, obscenely overpaid and sporting a brand-new MBA. From my first day, however, when I was told that some colleagues hadn't really wanted to see me hired, I found myself in the middle of a venal greed machine whose story unfolded with Kafkaesque absurdity and 
frustration. Working for the railway meant cocky wheeling and dealing, and the insidious groupthink that made railway employees unquestioningly accept spoon-fed propaganda [...] Over the course of my public life, I have insisted-I believe correctlythat people, regardless of their position or power, take responsibility for their conduct. I can and will ask no less of myself. For this reason, I am resigning from the office of railway CEO [...].

\section{Narrative denial}

[...] The bigger they are, the harder they fall. I have been given much - the love of my family, the faith and trust of the people of this country, and the chance to lead this company. It hurts that these joys are now taken away from me, although I did not do anything wrong. To every train passenger-and to all those who believed in what I tried to stand forthe accusation is false. I am not guilty of transferring funds to my private account. I deny everything I was accused of over the last few days.

When I first entered the railway's office complex, I was the epitome of the classic manager: young, brash, obscenely overpaid and sporting a brand-new MBA. From my first day, however, when I was told that some colleagues hadn't really wanted to see me hired, I found myself in the middle of a venal greed machine whose story unfolded with Kafkaesque absurdity and frustration. Working for the railway meant cocky wheeling and dealing, and the insidious groupthink that made railway employees unquestioningly accept spoon-fed propaganda [...] Over the course of my public life, I have insisted-I believe correctlythat people, regardless of their position or power, should not stay on when they feel no longer wanted. I can and will ask no less of myself. For this 
reason, I am resigning from the office of railway CEO [...].

\section{Study 3}

High empathy

[...] I want to apologize for the experience you are referring to. I feel really unhappy about the way I treated you, under any circumstance and I certainly understand why you aren't happy either. Please allow me to present my side of the story so that I can, I hope, make you understand where I was coming from [...] I felt frustrated and when I heard your price, the first thing I thought was: "This price competition in our industry is killing me. How can I satisfy my customers, when the price is never right?" So, when you were seated in the room and I disappeared for a couple of minutes, I was really trying to convince the manager why you weren't willing to pay that much for the car. He said to me that you could get it for a little more than what you were offering. But we both know that it's easy to get great deals practically around the corner in this industry. Indeed, I hear of a new competitive move almost every day, so I feel the only way I can improve myself as a sales representative is listening carefully to you, my customer, so that I can think of ways to add value [...] I totally understand that you found my offer unsatisfactory. It feels the same to me. All I ask from you is to not condemn me for this one frustrating experience [...]. 
Low empathy

[...] First off, let us express our apologies for the experience you are referencing. We are not happy with the way you were treated, under any circumstance, and it is certainly clear why you aren't either. We would like to take a moment to present our side of the story for your information, although we will not be held liable [...] Now, when you told the sales representative your price, he arrived at the conclusion that this price competition in the industry is a killer and that it is a major roadblock to achieving customer satisfaction here at R\&W Cars. So, when you were seated in the room and the sales representative disappeared for a couple of minutes, he was trying to convince the manager why you weren't willing to pay that much for the car. The manager told him that you could get it for a little more than what you were offering. However, it is an undeniable fact that it's easy to get great deals practically around the corner in this industry. One hears of a new competitive move almost every day, so that is why we decided to focus on added value as our competitive edge [...] To us it is clear that you found the offer unsatisfactory. However, do not condemn the entire organization for this one frustrating experience [...].

\section{THE MEANS TO JUSTIFY THE END}

\section{Study 1}

Narrative cyber harassment report

[...] I'd like to tell you about a cyber harassment episode-how it has influenced me-by presenting my side of the story. About two months ago, Adam Klein took a ridiculous picture of me. He had a "fan" 
page on the social network site set up about me, and this picture was posted on it. Adam aimed the page at me, writing my full name in capitals and everything, so everyone who read it knew it was me. Adam sent me rude and demeaning messages and mean comments. He also turned his contacts against me by telling lies and posting rude comments on the social network [...] Later, some other users put hurtful things on the "fan" page. With no access to the account, I had no way to get the posts removed. It hurt a lot to read these things, and it caused me a lot of stress. I felt that I was openly being ridiculed, because people were having a joke at my expense that I could not stop. It was a horrible situation and really made me sick. It affected my functioning so much that I lost confidence and was upset and emotional about being a source of ridicule. I was also worried about the response from my family if they saw the picture [...].

Analytical cyber harassment report

[...] As you are aware, article 5 in the terms of use of the social network regards the issue of user behaviour on profile pages. It states that cyber harassment "involves the use of the social network to support deliberate, repeated, and hostile behaviour by an individual or group of users that is intended to harm other users."

Researchers from the University of Wisconsin have demonstrated a number of serious consequences of cyber harassment victimization. Victims

(a) have lower self-esteem.

(b) feel anxious, frustrated, and depressed. 
(c) avoid other users (often the very intention of the cyber bully).

(d) form an idea of and/or commit suicide.

There are at least four examples in Europe where cyber harassment has been linked to the suicide of a social media user [...].

Network provider response

[...] Episodes of cyber harassment have a very negative impact on the victimized users. The social network believes something must be done to address this problem. We have decided that the network provider is best suited to determine what steps should be taken to rectify the problem [...] We have decided that no response would just result in continued inappropriate user behaviour. Thus, we have decided to restrict the use of profanity in status updates, comments, etc. and to discontinue user accounts that are deemed in violation. We hope this decision will help the social network regain its status as a network with users that make us proud.

\section{Study 2}

\section{Network provider response}

As you may be aware, in the past month there has been an alarming trend amongst [university name]-Network users. There have been serious issues related to the appearance of offending groups and the use of inappropriate speech among members. As you may know, we at the [university name]-Network strive for a respectful atmosphere in our alumni network. 
Personal identity violation

Therefore, following the guidelines in our regulations, we decided to remove all your personal information concerning faculties, studies, and student life. From now on, you are no longer allowed to have any information about your faculty, study, or student association on your personal [university name]-Network page. The [university name]-Network also must grant permission for you to make any change to your personal profile website. Furthermore, we decided to introduce a "cooling-off" period of 30 days in which you are not allowed to send messages or post updates from your [university name]-Network account. We hope that with this decision, mutual respect amongst [university name]-Network users returns.

\section{Social identity violation}

Therefore, following the guidelines in our regulations, we decided to remove all information of all students concerning faculties, studies, and student life. From now on, it is no longer allowed for students to have any information about their faculty, study, or student association on their [university name]-Network page. The [university name]-Network also must grant permission for any change to students' profile websites. Furthermore, we decided to introduce a "cooling-off" period of 30 days in which no student is allowed to send messages or post updates from their [university name]-Network account. We hope that with this decision, mutual respect amongst [university name]-Network users returns. 


\section{Measures}

\section{Absorption}

(1) The sound of a voice can be so fascinating to me that I can just go on listening to it.

(2) While watching in a play, I have sometimes really felt the emotions of the character and have "become" him (her) for the time being, forgetting, as it were, both myself and the audience.

(3) I can sometimes recollect certain past experiences in my life with such clarity and vividness that it is like living them again or almost so.

(4) If I wish, I can imagine (or daydream) some things so vividly that they hold my attention in the way a good movie or story does.

(5) I can tell a story with elaborations to make it sound better and then have the elaboration seem as real to me as the actual incident, or almost so.

(6) I am sometimes able to forget about my present self and get absorbed in a fantasy that I am someone else.

(7) If I wish, I can imagine that my body is so heavy that I could not move it if I wanted to.

(8) I enjoy-or would enjoy-getting beyond the world of logic and reason to experience something new and different.

(9) It gives me-or would give me-deep satisfaction to devote myself to someone I care about.

(10) I would much rather stick to my own ideas than be guided by others. (reversed)

\section{Concern for reputation}

(1) I am rarely concerned about my reputation.

(2) I do not consider what others say about me.

(3) I wish to have a good reputation

(4) If my reputation is not good, I feel very bad.

(5) I find it important that others consider my reputation as a serious matter.

(6) I try hard to work on my reputation (in my relationships with others).

(7) I find it difficult if others paint an incorrect image of me. 


\section{Customer orientation}

(1) I try to figure out what customer needs are.

(2) A good employee has to have the customer's best interest in mind.

(3) I try to bring a customer with a problem together with a product/service that helps solve that problem.

(4) I offer the product/service that is best suited to the customer's problem.

(5) I try to find out what kind of products/services will be most helpful to a customer.

\section{Distributive justice}

(1) I feel the network provider's decision was fair.

(2) The network provider's decision reflects the committed offence.

(3) The network provider's decision is appropriate for the committed offence.

(4) The network provider's decision reflects what has been done to the social-networking site.

(5) The network provider's decision is justified, given the committed offence.

(6) Overall, I feel the network provider's decision was unfair. (reversed)

\section{Empathic ability}

(1) I daydream and fantasize, with some regularity, about things that might happen to me.

(2) I really get involved with the feelings of the characters in a novel.

(3) I am usually objective when I watch a movie or play, and I don't often get completely caught up in it. (reversed)

(4) Becoming extremely involved in a good book or movie is somewhat rare for me. (reversed)

(5) After seeing a play or movie, I have felt as though I were one of the characters.

(6) When I watch a good movie, I can very easily put myself in the place of a leading character.

(7) When I am reading an interesting story or novel, I imagine how I would feel if the events in the story were happening to me. 


\section{Empathy}

(1) I could daydream and fantasize about the things that happened to [author name].

(2) I really got involved with the feelings of [author name].

(3) I was objective while reading the blog post, and I did not get completely caught up in it. (reversed)

(4) I did not get extremely involved in the blog post. (reversed)

(5) After reading the blog post, I felt as though I were [author name].

(6) While I was reading the blog post, I could very easily put myself in the place of [author name].

(7) While I was reading the blog post, I imagined how I would feel if the events in the story were happening to me.

\section{Format}

The post featured...

(1) a well-documented analysis by [name author] of what happened-a personal story told from [name author]'s point of view

(2) arguments-images

(3) a line of logic-a chain of events

(4) evidence-context

(5) professional expertise-personal experience

(6) reasoning-suspense

(7) information-emotion 


\section{Image-producing capacity}

Think of some relative or friend whom you frequently see, considering carefully the picture that comes to mind, and classify the image suggested as indicated by the degrees of clearness and vividness specified:

(1) With respect to the exact contour of face, head, shoulders, and body, the image which comes to my mind is...

(2) With respect to characteristic poses of head, attitudes of body, etc., the image which comes to my mind is...

(3) With respect to the precise carriage, length of step, etc., in walking, the image which comes to my mind is...

(4) With respect to the different colours worn in some familiar costume, the image which comes to my mind is...

Think of the sun as it is sinking below the horizon, considering carefully the picture that comes to mind, and classify the image suggested as indicated by the degrees of clearness and vividness specified:

(5) With respect to the mental picture suggested, the image which comes to my mind is...

\section{Intention to switch}

(1) I would not let Dr. Jacobs have any influence over my own or my partner's health.

(2) I would be comfortable asking Dr. Jacobs to cure a serious illness, even if I could not monitor his actions. (reversed)

(3) I would be comfortable having Dr. Jacobs make decisions that critically affect my own or my partner's health. (reversed)

(4) I would prefer another doctor to oversee Dr. Jacobs's decisions in my own or my partner's treatment.

(5) If I or my partner had to see an obstetrician, I would rather go to another doctor than to Dr. Jacobs.

(6) I would keep an eye out for another doctor. 


\section{Love of money}

(1) Money is a symbol of success.

(2) Money will help you express your competence and abilities.

(3) Money represents one's achievement.

(4) I value money very highly.

(5) Money makes people respect you in your community.

(6) Money can give you the opportunity to be what you want to be.

(7) Money gives you autonomy and freedom.

(8) Money is important.

\section{Moral attentiveness}

(1) In a typical day, I face several ethical dilemmas.

(2) I often have to choose between doing what's right and doing something that's wrong.

(3) I regularly face decisions that have significant ethical implications.

(4) My life has been filled with one moral dilemma after another.

(5) Many of the decisions that I make have ethical dimensions to them.

(6) I regularly think about the ethical implications of my decisions.

(7) I think about the morality of my actions almost every day.

(8) I rarely face ethical dilemmas. (reversed)

(9) I frequently encounter ethical situations.

(10) I often find myself pondering about ethical issues.

(11) I often reflect on the moral aspects of my decisions.

(12) I like to think about ethics. 


\section{Need for cognition}

(1) I would prefer complex to simple problems.

(2) I like to have the responsibility of handling a situation that requires a lot of thinking.

(3) Thinking is not my idea of fun. (reversed)

(4) I would rather do something that requires little thought than something that is sure to challenge my thinking abilities.(reversed)

(5) I try to anticipate and avoid situations where there is likely a chance I will have to think in depth about something.(reversed)

(6) I find satisfaction in deliberating hard and for long hours.

(7) I only think as hard as I have to.(reversed)

(8) I prefer to think about small, daily projects to longterm ones.(reversed)

(9) I like tasks that require little thought once I've learned them.(reversed)

(10) The idea of relying on thought to make my way to the top appeals to me.

(11) I really enjoy a task that involves coming up with new solutions to problems.

(12) Learning new ways to think doesn't excite me very much.(reversed)

(13) I prefer my life to be filled with puzzles that I must solve.

(14) The notion of thinking abstractly is appealing to me.

(15) I would prefer a task that is intellectual, difficult, and important to one that is somewhat important but does not require much thought.

(16) I feel relief rather than satisfaction after completing a task that required a lot of mental effort.(reversed)

(17) It's enough for me that something gets the job done; I don't care how or why it works.(reversed)

(18) I usually end up deliberating about issues even when they do not affect me personally.

\section{Perceived integrity}

(1) I like [character name]'s values.

(2) Sound principles seem to guide [character name]'s behaviour.

(3) [character name] will stick to his word.

(4) [character name] has a great deal of integrity. 


\section{Procedural justice}

(1) I consider the way in which I have been treated fair.

(2) I consider the way in which I have been treated just.

(3) I consider the way in which I have been treated justified.

\section{Self-esteem}

(1) How proud are you of yourself?

(2) How positive do you think of yourself?

(3) How much do you value yourself?

(4) To what extent do you feel less than others?

\section{Selling orientation}

(1) I try to sell as much as I can rather than to satisfy a customer.

(2) It is necessary to stretch the truth in describing a product to a customer.

(3) I try to sell a customer all I can convince them to buy, even if I think it is more than a wise customer would buy.

(4) I paint too rosy a picture of my product/service to make them sound as good as possible.

(5) I decide what product/service to offer on the basis of what I can convince customers to accept, not on the basis of what will satisfy them in the long run.

\section{Social identification}

(1) Being a social network user is a big part of my identity.

(2) Being a social network user is important to me.

(3) I feel proud to be a part of a social network. 


\section{Transportation}

(1) While I was reading the post, I could easily picture the events in it taking place.

(2) While I was reading the post, activity going on in the room around me was on my mind. (reversed)

(3) I could easily picture myself in the scene of the events described in the post.

(4) I was mentally involved in the post while reading it.

(5) After finishing the post, I found it easy to put it out of my mind. (reversed)

(6) I wanted to learn how the post ended.

(7) The post affected me emotionally.

(8) I found myself thinking of ways the post could have turned out differently.

(9) I found my mind wandering while reading the post. (reversed)

(10) The events in the post are relevant to my everyday life.

(11) The events in the post have changed my life.

(12) While reading the post, I had a vivid image of [name character].

\section{Willingness to respond}

(1) I am willing 'to go the extra mile' to retain Paul as a customer.

(2) I feel loyal toward Paul. 


\section{SUMMARIES}

RETURN OF THE NARRATIVE

\section{LOST IN TRANSPORTATION?}

Transportation refers to a phenomenon by which readers mentally enter a world evoked by a narrative, which may constitute the dominant source of narrative persuasion. To establish a clearer understanding of this effect, the author reviews 10 years of transportation and narrative persuasion studies published in four different languages and identifies four outcomes and five potential moderators in a meta-analytic random effects model. The findings indicate positive transportation effects for medium ranges of affective responses, belief changes, and behavioural intents. The effect on cognitive responses is small but positive. Furthermore, several attributes of the reader, story, and medium, as well as empathy, mental imagery focus, and methodological features, moderate the transportation effect. These findings underscore the strategic role of narrative communication elements, study approach, methodology, and the increasing importance of transportation effects in public narratives. To explain variation in narrative persuasion, future data collections should span longer periods. 


\section{A WALK IN CUSTOMERS' SHOES}

The number of weblog (blog) posts that expose company integrity violations has increased dramatically. In response, some companies allow employees to engage in direct dialogue with bloggers, which requires employees to take the customer's perspective before responding. Narrative transportation, or the extent to which employees mentally enter the world evoked by a customer's story, affects their inclination to assume ownership of an integrity violation. An extensive field study primes branch managers of a global Fortune 100 banking group with a self-sufficient, cognitive, or empathic perspective. The results indicate that transportation mediates the effect of perspective taking on two relevant psychological ownership dimensions: acknowledgment of responsibility and willingness to respond. Participants with a selfsufficient or cognitive perspective neither acknowledge responsibility nor want to respond. However, participants primed with an empathic perspective take responsibility for the customer's case and respond to the integrity violation. The effect strengthens when the employee perceives the customer's financial vulnerability as high. 


\section{IN STORIES WE TRUST}

Consumers' confidence in companies has fallen due to recent and widespread violations of integrity and consumers' voicing of discontent in weblog (blog) posts. Current research on integrity restoration offers little guidance regarding appropriate responses. The author posits that not only what (with which content) but also how (in which format) the company responds, contributes to an effective restoration of integrity and a reduction of consumers' intentions to switch. The results of Study 1 show that the combination of denial content and analytical format as well as apologetic content and narrative format works better than combinations of opposing response content and format. Comparing narrative apologies and denials in two consecutive studies, the author demonstrates that the concept of "transportation"the engrossing effect of a narrative-is the mechanism underlying narrative-based integrity restoration. The author further assesses in Study 2 how the use of empathy accounts for higher levels of transportation and perceived integrity. In Study 3 , the author establishes that a personal response by the involved employee is more effective than a response issued by the company's spokesperson. Consumers trust in stories from the involved employee. 


\section{THE MEANS TO JUSTIFY THE END}

Social media users' perceptions of privacy regulation decisions depend on whether they become engrossed in a narrative account (e.g., wall posts) that reports harassment. In particular, if a cyber harassment report is a narrative, rather than in an analytical format, responsive privacy regulation decisions prompt positive evaluations in terms of distributive and procedural justice. This impact likely arises because social media users consider the cyber harassment experience from the perspective of a fellow network member. A second study reveals that if the social network user relates the experience to his or her own profile, narrative transportation increases, which positively affects perceptions of the justice of privacy regulation decisions. Moreover, referencing the self increases narrative transportation even more, which again increases justice perceptions. 


\section{SAMENVATTINGEN}

TERUGKEER VAN HET VERHAAL

\section{VERDWAALD IN TRANSPORTATIE?}

Transportatie is een ander woord voor 'inleving in een verhaal'. Het is één van de belangrijkste bronnen van overtuigingskracht van verhalen. Om dit effect beter te begrijpen, bestudeert de auteur transportatie- en narratieve overtuigingsstudies die de afgelopen tien jaar in vier verschillende talen gepubliceerd werden. Hij stelt vier uitkomsten en vijf potentiële moderators vast in een metaanalytisch random effects model. Zo blijkt dat transportatie positieve, middelgrote effecten heeft op affectieve reacties, overtuiging, en gedragsmatige intenties. Het effect op cognitieve reacties is klein, maar positief. De kracht van transportatie wordt bovendien beïnvloed door verschillende eigenschappen van de lezer, het verhaal en het medium, evenals de mate van empathie en verbeelding en methodologische kenmerken. Deze bevindingen onderstrepen de strategische rol van deze narratieve communicatieelementen, studieaanpak en methodologie, alsook het toenemende belang van transportatie-effecten in publieke verhalen. Om de variatie in narratieve overtuiging te verklaren, is vervolgonderzoek met een langere tijdspanne noodzakelijk. 


\section{IN DE SCHOENEN VAN DE KLANTEN}

Het aantal (we)blogberichten over het gebrek aan vertrouwen in bedrijven is sinds het begin van de economische crisis in 2007 dramatisch toegenomen. Als antwoord hierop zijn bedrijven aan het experimenteren met rechtstreeks contact tussen hun medewerkers en bloggende klanten. Hierbij is het belangrijk dat werknemers zich voorstellen in de schoenen van de klant te staan, voordat ze reageren. Narratieve transportatie, oftewel de mate waarin medewerkers zich inleven in het verhaal (d.w.z. de ervaring) van een klant, heeft invloed op de mate waarin ze zich verantwoordelijk voelen voor de klacht van een klant. In een uitgebreid veldonderzoek heeft de auteur bij filiaalmanagers van een financiële Fortune 100 multinational ofwel een op het eigen belang gericht, een cognitief, of een empathisch perspectief geactiveerd (geprimed). Het blijkt dat een dergelijk perspectief effect heeft op de verantwoordelijkheid die men neemt voor het belang van de klant en ook de bereidheid om te reageren. Deelnemers die het eigen belang voorop stellen of reageren vanuit een cognitief perspectief erkennen geen verantwoordelijkheid en hebben niet de wil om te reageren. De deelnemers geprimed met een empathisch perspectief nemen wél verantwoordelijkheid voor de situatie waarin de klant zich bevindt en zijn bereid om te helpen. Dit effect loopt via transportatie. Tenslotte vindt hij dat in het geval dat de financiële kwetsbaarheid van de klant als hoog wordt ingeschat, het effect wordt versterkt. 


\section{EEN BETROUWBAAR VERHAAL}

Het vertrouwen van consumenten in bedrijven lijkt tot een dieptepunt gedaald. Steeds vaker uiten consumenten hun ongenoegen via (we)blogs. Het meest recente wetenschappelijk onderzoek naar vertrouwensherstel biedt weinig houvast hoe bedrijven het meest effectief kunnen reageren op deze nieuwe vorm van interactie. De auteur stelt dat niet alleen de inhoud waarmee, maar ook de vorm waarin een bedrijf reageert, bijdraagt aan een effectief herstel van het vertrouwen en een vermindering van de intentie van de consument om naar de concurrent over te stappen. Uit de resultaten van studie 1 blijkt dat de combinatie van een ontkennende inhoud in analytische vorm en een verontschuldigende inhoud in narratieve vorm beter werkt dan vice versa. Door narratieve verontschuldigingen en ontkenningen te vergelijken in twee vervolgstudies, laat de auteur zien dat transportatie - inleving in een verhaal ten grondslag ligt aan de meest effectieve vorm van reageren. De auteur kijkt in studie 2 hoe het gebruik van empathie tot een hoger niveau van transportatie en vertrouwen leidt. In studie 3 stelt hij vast dat een persoonlijke reactie van een werknemer effectiever is dan een reactie van een woordvoerder. Een betrouwbaar verhaal is dan ook een verhaal van de betrokken werknemer. 


\section{DE MIDDELEN OM HET DOEL TE HEILIGEN}

Het oordeel van gebruikers van sociale media over de gehanteerde privacyregelgeving binnen die sociale media wordt sterk beïnvloed door de mate waarin meldingen van intimidatie verhalend worden beschreven (in dit geval in prikbordberichten). Dat blijkt uit twee studies. Ook hier is het effect van narratieve transportatie onderzocht. Als een verslag van cyberintimidatie narratief (verhalend) is, blijken andere gebruikers daaropvolgende besluiten over privacyregelgeving positiever te beoordelen in termen van distributieve en procedurele rechtvaardigheid dan als het verslag een analytische opbouw heeft. De auteur geeft als verklaring dat de sociale mediagebruiker de cyberintimidatie-ervaring vanuit het perspectief van een netwerkgenoot ziet. Een tweede studie vindt dat als de sociale netwerkgebruiker ervaart dat zijn online profiel geïntimideerd wordt, transportatie hoger is en dit een positief effect heeft op het oordeel over besluiten over striktere privacyregulering. Als de gebruiker ervaart dat zijn eigen ik geïntimideerd wordt, versterkt dat de transportatie, wat vervolgens weer het gevoel van rechtvaardigheid over striktere privacyregulering verhoogt. 

